\title{
Consenso de manejo del paciente con diabetes mellitus y patología cardiovascular
}

\section{Sociedad Argentina de Cardiología (SAC)- Sociedad Argentina de Diabetes (SAD)}

\section{Consensus on the management of patients with diabetes mellitus and cardiovascular disease}

\section{Argentine Society of Cardiology-Argentine Society of Diabetes}

\section{Trabajo realizado en conjunto por ambas Sociedades Científicas}

Directores: Mariano Aníbal Giorgi (SAC), León E. Litwak (SAD)

Co-Director: Hugo Sanabria (SAC)

Secretarios: Augusto Lavalle Cobo (SAC), Carla Musso (SAD), Emiliano Salmeri (SAC)

Autores: José Álvarez' ${ }^{1}$, Florencia Aranguren², Fernando Belcastro ${ }^{3}$, Fernando Brites ${ }^{4}$, Carlos Buso ${ }^{5}$, Marta Calvagno $^{6}$, Christian Caroli7, Leonardo Celano ${ }^{8}$, Hernán Cohen Arazi ${ }^{9}$, Daniel Comtesse ${ }^{10}$, Pablo Corral ${ }^{11}$, Juan Pablo Costabel ${ }^{12}$, Alicia Elbert ${ }^{13}$, Cristina Faingold ${ }^{14}$, Graciela Fuente ${ }^{15}$, Pablo García Merletti ${ }^{16}$, Mariano Aníbal Giorgi ${ }^{17}$, Gustavo Giunta ${ }^{18}$, Héctor Gómez Santamaría ${ }^{19}$, Enrique González Naya ${ }^{20}$, Claudio Hadid ${ }^{21}$, Ariel Kraselnik ${ }^{22}$, Augusto Lavalle Cobo ${ }^{23}$, León Litwak ${ }^{24}$, Martín Lobo ${ }^{25}$, Alfredo Lozada ${ }^{26}$, Claudio Majul ${ }^{27}$, Walter Masson ${ }^{28}$, Carla Musso ${ }^{29}$, Juan Nogueira ${ }^{30}$, Gabriel Persi ${ }^{31}$, Martín Rodríguez ${ }^{32}$, Emiliano Salmeri $^{33}$, Hugo Sanabria ${ }^{34}$, Silvio Schraier ${ }^{35}$, Isaac Sinay ${ }^{36}$, Daniel Siniawski ${ }^{37}$, Jorge Thierer ${ }^{38}$

\section{Revisores:}

Por la Sociedad Argentina de Diabetes:

Susana Salzberg ${ }^{39}$, Cristian Suárez Cordo ${ }^{40}$

Por la Sociedad Argentina de Cardiología

Jorge Lerman ${ }^{41}$, Álvaro Sosa Liprandi²

\section{RESUMEN}

Este Consenso sale a la luz en medio de una de las peores crisis sanitarias globales de los últimos 100 años. EI SARS-CoV-2 y su manifestación clínica, la COVID-19, han provocado una disrupción en cómo médicos y pacientes nos relacionamos. Si bien se trata de una enfermedad infecciosa, una de las características más notables es que su mortalidad se acrecienta en pacientes con enfermedades crónicas no transmisibles $y$, en particular, con antecedentes de diabetes y enfermedad cardiovascular. En tal contexto, entonces, creemos que cobran más relevancia las recomendaciones vertidas en este documento, que apuntan a identificar y proteger a estos pacientes, al tiempo que se vuelve prioritaria la implementación, más allá de los enunciados, de políticas concretas de prevención cardiometabólica. Metodología: este consenso es el fruto de la voluntad de dos Sociedades Científicas que han reconocido la necesidad de

\section{ABSTRACT}

This Consensus comes to light in the midst of one of the worst global health crises in the last 100 years. SARS-CoV-2 and its clinical manifestation, COVID-19, have caused a disruption in how doctors and patients interact. Although it is an infectious disease, one of the most notable characteristics is that its mortality increases in patients with chronic non-communicable diseases and, in particular, with a history of diabetes and cardiovascular disease.

In this context, then, we believe that the recommendations made in this document, which aim to identify and protect these patients, become more relevant, while the implementation, beyond the statements, of specific cardiometabolic prevention policies becomes a priority.

Methodology: this consensus is the result of the will of two Scientific Societies that have recognized the need to comple- 
Revista de la Sociedad Argentina de Diabetes Año 55 Vol. 55 № 1 Suplemento Consenso Sociedad Argentina de CardiologíaSociedad Argentina de Diabetes Enero-abril de 2021: 03-70 ISSN 0325-5247 (impresa) ISSN 2346-9420 (en línea)

complementar el enfoque sobre una misma problemática: la de los pacientes con diabetes mellitus (DM) y con enfermedad cardiovascular (ECV), o bien que están en riesgo de sufrirla. Tanto la Sociedad Argentina de Cardiología (SAC) como la Sociedad Argentina de Diabetes (SAD) tienen una reconocida trayectoria en la producción de guías de práctica y documentos de consenso, aunque cada una tiene prácticas y culturas de trabajo diferentes. En consecuencia, la primera tarea que se afrontó fue la de acordar, no solamente el temario y el abordaje de los diferentes asuntos, sino también modalidades de trabajo comunes: objetivo general del documento, forma de analizar y evaluar el peso de la información, definir los niveles de evidencia y determinar los grados de recomendación. Se acordó adoptar la modalidad utilizada por la SAC en todos los documentos producidos por el Área de Consensos y Normas, expuestos en el siguiente cuadro:

\section{Grado de recomendación}

- Clase I: condiciones para las cuales hay evidencia y/o acuerdo general en que el tratamiento/procedimiento es beneficioso, útil y eficaz.

- Clase II: evidencia conflictiva y/o divergencia de opinión acerca de la utilidad, eficacia del método, procedimiento y/o tratamiento.

- Ila: el peso de la evidencia/opinión está a favor de la utilidad/eficacia.

- Ilb: la utilidad/eficacia está menos establecida.

- Clase III: evidencia o acuerdo general que el tratamiento método/procedimiento no es útil/eficaz y en algunos casos puede ser perjudicial.

\section{Nivel de evidencia}

- A: evidencia sólida, proveniente de estudios clínicos aleatorizados o de cohortes con diseño adecuado para alcanzar conclusiones estadísticamente conectadas y biológicamente significativas.

- B: datos procedentes de un único ensayo clínico aleatorizado o de grandes estudios no aleatorizados.

- C: consenso de opinión de expertos.

Los expertos que colaboraron en la redacción del Consenso fueron seleccionados e invitados a participar con el acuerdo unánime del grupo de Directores y Secretarios pertenecientes a ambas Sociedades Científicas. Se convocó a colegas con reconocida trayectoria en las disciplinas abordadas para el análisis de la evidencia y la redacción de las recomendaciones. Todos los aspectos metodológicos y las recomendaciones finales de este documento fueron definidos por acuerdo entre el grupo de Directores y Secretarios del Consenso.

El proceso de consolidación de la información fue lento: desde la decisión de ambas sociedades hasta la redacción de este documento, el campo del manejo de la DM y la ECV sufrió profundas transformaciones que trascienden la aparición de nuevos agentes terapéuticos. Lo que se ha desarrollado es un nuevo modelo de abordaje que es, según las palabras de la Dra. Alicia Elbert, transdisciplinario. Esto ha implicado esperar y poder entender y "procesar" toda la información surgida en estos años. Este documento, que pretende asistir a los médicos en la práctica diaria, ha intentado adoptar esa nueva mirada integradora.

Palabras clave: guías; diabetes mellitus; enfermedad cardiovascular; epidemiología; factores de riesgo; prevención; valoración de riesgo cardiovascular; tratamiento farmacológico; revascularización; insuficiencia cardíaca.

Revista de la Sociedad Argentina de Diabetes 2021; Vol. 55 (03-70) ment the focus on the same problem: that of patients with diabetes mellitus (DM) and cardiovascular disease (CVD), or who are at risk to suffer it. Both the Argentine Society of Cardiology (SAC) and the Argentine Society of Diabetes (SAD) have a recognized track record in the production of practice guidelines and consensus documents, although each has different work practices and cultures. Consequently, the first task that was faced was to agree, not only on the agenda and the approach to the different issues, but also on common working methods: general objective of the document, how to analyze and evaluate the weight of the information, define the levels of evidence and determine the degrees of recommendation. It was agreed to adopt the modality used by the SAC in all the documents produced by the Consensus and Standards Area, set out in the following table:

\section{Grade of recommendation}

- Class I: conditions for which there is evidence and/or general agreement that the treatment/procedure is beneficial, useful and effective.

- Class II: conflicting evidence and/or divergence of opinion about the usefulness, efficacy of the method, procedure and / or treatment.

- Ila: the weight of evidence/opinion is in favor of utility/ efficacy.

- Ilb: utility/efficacy is less established.

- Class III: evidence or general agreement that the treatment method/procedure is not useful/effective and in some cases may be harmful.

\section{Level of evidence}

- A: solid evidence, from randomized clinical studies or from cohorts with adequate design to reach statistically connected and biologically significant conclusions.

- B: data from a single randomized clinical trial or large nonrandomized studies.

- C: consensus of expert opinión.

The experts who collaborated in the drafting of the Consensus were selected and invited to participate with the unanimous agreement of the group of Directors and Secretaries belonging to both Scientific Societies. Colleagues with recognized experience in the disciplines addressed were summoned to analyze the evidence and write the recommendations. All the methodological aspects and the final recommendations of this document were defined by agreement between the group of Directors and Consensus Secretaries.

The information consolidation process was slow: from the decision of both companies until the writing of this document, the field of DM and CVD management underwent profound transformations that transcend the appearance of new therapeutic agents. What has been developed is a new approach model that is, in the words of Dr. Alicia Elbert, transdisciplinary. This has implied waiting and being able to understand and "process" all the information that has emerged in these years. This document, which aims to assist physicians in daily practice, has tried to adopt this new integrative perspective.

Key words: guidelines; diabetes mellitus; cardiovascular diseases; epidemiology; risk factors; prevention; cardiovascular risk assessment; patient management; pharmacological treatment; revascularization; heart failure.

Revista de la Sociedad Argentina de Diabetes 2021; Vol. 55 (03-70) 
Revista de la Sociedad Argentina de Diabetes Año 55 Vol. 55 № 1 Suplemento Consenso Sociedad Argentina de CardiologíaSociedad Argentina de Diabetes Enero-abril de 2021: 03-70 ISSN 0325-5247 (impresa) ISSN 2346-9420 (en línea)

1 Cardiólogo, Jefe del Servicio de Hemodinamia y Cardioangiología Intervencionista, Hospital Alemán, Ciudad Autónoma de Buenos Aires, Argentina

2 Especialista en Medicina Interna, especializada en Diabetes, Directora de la Diplomatura en Riesgo Cardiometabólico y Renal, Universidad de Ciencias Empresariales y Sociales (UCES), Ciudad Autónoma de Buenos Aires, Argentina

3 Médico Cardiólogo, Jefe del Programa de Medicina Vascular, Instituto Cardiovascular de Buenos Aires (ICBA), Ciudad Autónoma de Buenos Aires, Argentina

4 Doctor en Bioquímica especialista en Lípidos, Lipoproteínas y Aterosclerosis, Miembro del Laboratorio de Lípidos y Lipoproteínas, Departamento de Bioquímica Clínica, Hospital de Clínicas "José de San Martín", Universidad de Buenos Aires, Ciudad Autónoma de Buenos Aires, Argentina

5 Especialista en Medicina Interna, Nutrición y Diabetes, especialista en Cardiología, Grupo de Trabajo Nutrición y Diabetes, Hospital Municipal de Oncología Marie Curie, Consultorio Integral Dibetológico (Morón, Buenos Aires), Provincia de Buenos Aires, Argentina

6 Médica especialista en Nutrición y Diabetes, Profesora adjunta Cátedra de Nutrición, Universidad de Buenos Aires (UBA), Ciudad Autónoma de Buenos Aires, Argentina

7 Médico Cardiólogo, miembro titular de la Sociedad Argentina de Cardiología, Ex subdirector de la Unidad Médica Presidencial Argentina (2015-1019), staff médico del Hospital Médica MIA, Estado de México, México

8 Cardiólogo, staff de Electrofisiología, Hospital Cosme Argerich y del Centro de Educación Médica e Investigaciones Clínicas "Norberto Quirno" (CEMIC), Ciudad Autónoma de Buenos Aires, Argentina

9 Cardiólogo, Universidad de Buenos Aires (UBA), Magister en Efectividad Clínica y Sanitaria (UBA), Coordinador de Unidad Coronaria, Sanatorio Trinidad Mitre, Ciudad Autónoma de Buenos Aires, Argentina

10 Médico Cardiólogo, Sección de Cardiometabolismo y Enfermedades Aterotrombóticas, Instituto Cardiovascular San Isidro, Sanatorio Las Lomas, Miembro del Consejo de Cardiometabolismo de la Sociedad Argentina de Cardiología, Provincia de Buenos Aires, Argentina

11 Especialista en Medicina Interna, Miembro titular de la Sociedad Argentina de Cardiología, Docente de la Carrera de Medicina, Universidad Fraternidad de Agrupaciones Santo Tomás de Aquino (FASTA), Mar del Plata, Provincia de Buenos Aires, Argentina

12 Especialista Universitario en Cardiología, Universidad de Buenos Aires (UBA), Jefe de la Unidad Coronaria, Instituto Cardiovascular de Buenos Aires (ICBA), Ciudad Autónoma de Buenos Aires, Argentina

13 Médica Nefróloga especializada en Diabetes, Directora del Centro de Enfermedad Renal y Hipertensión Arterial (CEREHA), Ciudad Autónoma de Buenos Aires, Argentina

14 Responsable Médico, Servicio de Endocrinología, Unidad Asistencial Dr. César Milstein, Médica de Planta, Instituto Cardiovascular de Buenos Aires (ICBA), Directora de la Carrera de Médico Especialista en Endocrinología Universidad de Buenos Aires (UBA), Presidente de la Sociedad Argentina de Diabetes 2015-2016, Ciudad Autónoma de Buenos Aires, Argentina

15 Médica especialista en Nutrición especializada en Diabetes, ex Jefa y actual Consultora de la Unidad de Nutrición, Hospital General de Agudos Carlos G. Durand, Ciudad Autónoma de Buenos Aires, Argentina

16 Cardiólogo Universitario, Director de la Carrera de Medicina de la Universidad del Salvador (USAL), Ciudad Autónoma de Buenos Aires, Argentina
17 Cardiólogo, Universidad de Buenos Aires (UBA), Jefe de la Unidad de Prevención Cardiovascular, Sección Cardiología, Centro de Educación Médica e Investigaciones Clínicas "Norberto Quirno" (CEMIC), Profesor Asociado de Farmacología, Instituto Universitario CEMIC, Ciudad Autónoma de Buenos Aires, Argentina

18 Médico Cardiólogo, Coordinador del Servicio de Cardiología Clínica, Hospital Universitario René G Favaloro, Fundación Favaloro, Universidad de Buenos Aires (UBA), Miembro Titular Sociedad Argentina de Cardiología, Ciudad Autónoma de Buenos Aires, Argentina

19 Cardiólogo, Coordinador de Cardiología, Sanatorio Finochietto, ex Director Consejo de Emergencias y Cardiología Crítica, Protesorero Sociedad Argentina de Cardiología 2021, Ciudad Autónoma de Buenos Aires, Argentina

20 Médico Cardiólogo, Jefe de Rehabilitación Cardiovascular, Instituto Argentino de Diagnóstico y Tratamiento (IADT), Miembro del Consejo de Ergometría, Rehabilitación Cardiovascular y Cardiología del Deporte, Sociedad Argentina de Cardiología, Ciudad Autónoma de Buenos Aires, Argentina

21 Cardiólogo, staff de Electrofisiología, Hospital Cosme Argerich y del Centro de Educación Médica e Investigaciones Clínicas "Norberto Quirno" (CEMIC), Ciudad Autónoma de Buenos Aires, Argentina

22 Especialista en Cardiología Clínica, Fundador de la Sociedad Argentina de Medicina de Estilo de Vida (SAMEV), Codirector Posgrado Nutrición Basada en Plantas, Universidad Nacional de Rosario, Miembro Sociedad Argentina de Cardiología, Rosario, Santa Fe, Argentina

23 Especialista en Cardiología, Coordinador del Servicio de Cardiología, Sanatorio Finochietto, ex director del Consejo Epidemiología y Prevención Cardiovascular de la Sociedad Argentina de Cardiología, Ciudad Autónoma de Buenos Aires, Argentina

24 Profesor Consulto del Instituto Universitario del Hospital Italiano de Buenos Aires, Médico Asociado al Servicio de Endocrinología, Metabolismo y Medicina Nuclear, Hospital Italiano de Buenos Aires, Ciudad Autónoma de Buenos Aires, Argentina

25 Jefe del Departamento Enfermedades Cardiovasculares y Cardiometabolismo, Hospital Militar Campo de Mayo, ex Director de Consejos Epidemiología y Prevención Vascular y Aterosclerosis y Trombosis, Sociedad Argentina de Cardiología, Provincia de Buenos Aires, Argentina

26 Cardiólogo, Jefe de la Clínica de Lípidos, Hospital Austral, Jefe de la Clínica de Lípidos de la Fundación para la Lucha contra las Enfermedades Neurológicas de la Infancia (FLE$\mathrm{NI}$ ), Ciudad Autónoma de Buenos Aires, Argentina

27 Médico Cardiólogo, Hospital Santojanni, Director del Consejo de Hipertensión Arterial (2014-2015), Vicepresidente $1^{\circ}$ de la Sociedad Argentina de Cardiología, Ciudad Autónoma de Buenos Aires, Argentina

28 Médico Cardiólogo, Jefe de Prevención Cardiovascular, Servicio de Cardiología, Hospital Italiano de Buenos Aires, Ciudad Autónoma de Buenos Aires, Argentina

29 Médica Endocrinóloga, Coordinadora de Diabetes, Fundación Favaloro, staff de Endocrinología, Unidad Asistencial César Milstein, Ciudad Autónoma de Buenos Aires, Argentina

30 Especialista en Endocrinología, Doctor en Nutrición, Investigador adjunto del Consejo Nacional de Investigaciones Científicas y Técnicas (CONICET), Presidente de la Sociedad Argentina de Lípidos, Ciudad Autónoma de Buenos Aires, Argentina

31 Neurólogo, Jefe del Área de Enfermedades Cerebrovasculares, Instituto de Neurociencias Buenos Aires (INEBA), Coordinador de Neurología, Sanatorio de la Trinidad Mitre, Ciudad Autónoma de Buenos Aires, Argentina 
Revista de la Sociedad Argentina de Diabetes Año 55 Vol. 55 № 1 Suplemento Consenso Sociedad Argentina de CardiologíaSociedad Argentina de Diabetes Enero-abril de 2021: 03-70 ISSN 0325-5247 (impresa) ISSN 2346-9420 (en línea)

32 Profesor Titular de Endocrinología, Metabolismo y Nutrición, Facultad de Ciencias Médicas, Universidad Nacional de Cuyo, Vicepresidente de la Sociedad Argentina de Diabetes, Mendoza, Argentina

33 Secretario Técnico del Consejo de Epidemiología y Prevención Cardiovascular y Vocal del Consejo de Cardiometabolismo, Sociedad Argentina de Cardiología, Cardiólogo Interno, Instituto Argentino de Diagnóstico y Tratamiento (IADT), Ciudad Autónoma de Buenos Aires, Argentina

34 Médico Cardiólogo, especializado en Diabetes, Jefe de la Clínica de Diabetes del Instituto Cardiovascular de Buenos Aires (ICBA), Ciudad Autónoma de Buenos Aires, Argentina

35 Especialista en Nutrición y Diabetes, Docente de Medicina Interna y Nutrición, Universidad de Buenos Aires (UBA), Director de la Carrera Especialización en Nutrición (UBA-sede HIBA), Vicedirector de la Carrera Especialización en Nutrición, Instituto Universitario de Ciencias de la Salud, Fundación Barceló, Ciudad Autónoma de Buenos Aires, Argentina

36 Médico asesor de la Clínica de Diabetes, Instituto Cardiovascular Buenos Aires (ICBA), Codirector del Departamento de Educación y Docencia de la Sociedad Argentina de Diabetes, Ciudad Autónoma de Buenos Aires, Argentina

37 Cardiólogo, Coordinador de la Clínica de Lípidos y Programa de Aféresis de Colesterol, Servicio de Cardiología, Hospital Italiano de Buenos Aires, Ciudad Autónoma de Buenos Aires, Argentina

38 Cardiólogo, Jefe de la Unidad Insuficiencia Cardíaca y Médico de la Unidad Coronaria, Centro de Educación Médica e Investigaciones Clínicas "Norberto Quirno" (CEMIC), Ciudad Autónoma de Buenos Aires, Argentina

39 Especialista en Nutrición y Diabetes, Directora del Departamento de Investigaciones Clínicas, Instituto Centenario, Ciudad Autónoma de Buenos Aires, Argentina

40 Especialista en Medicina Interna y Farmacología Clínica, especializado en Diabetes, Jefe del Área de Diabetes, Hospital Diego Thompson de San Martín, Provincia de Buenos Aires, Argentina

${ }^{41}$ Cardiólogo, Profesor Consulto de Cardiología, Facultad de Medicina, Universidad de Buenos Aires (UBA), ex Presidente de la Sociedad Argentina de Cardiología, ex Presidente de la Fundación Cardiológica Argentina, Ciudad Autónoma de Buenos Aires, Argentina

42 Cardiólogo, coordinador de Cardiología, Sanatorio Güemes, Director de la Residencia de Cardiología, Sanatorio Güemes, Ciudad Autónoma de Buenos Aires, Argentina

Contacto del autor: Mariano Giorgi

E-mail: marianoagiorgi@hotmail.com

Fecha de trabajo recibido: 29/12/20

Fecha de trabajo aceptado: 18/02/21
Conflictos de interés: El Dr. Hernán Cohen Arazi recibió honorarios como ponente de Roemmers y Servier. El Dr. Pablo Corral recibió honorarios como ponente para Amgen, Sanofi y Gador, financiación para una investigación de Amgen y como consultor de Amgen, Sanofi y Aegerion Pharmaceuticals. El Dr. Daniel Siniawski recibió honorarios como ponente de MSD, Sanofi y Novo Nordisk, y como consultor de MSD, Sanofi y Novo Nordisk. El Dr. Gustavo Giunta recibió honorarios como ponente de Laboratorio Raffo. El Dr. León Litwak recibió honorarios como ponente de Eli Lilly, Novo Nordisk, AstraZeneca, Roche, Janssen, Sanofi, Craveri, MSD, Boehringer Ingelheim y Abbott. El Dr. Emiliano Salmeri recibió honorarios como ponente de AstraZeneca. El Dr. Walter Masson recibió honorarios como ponente de Pfizer y Sanofi Aventis. El Dr. Augusto Lavalle Cobo recibió honorarios como ponente de AstraZeneca y como advisory board de Eurofarma y Bayer. El Dr. Fernando Belcastro recibió honorarios como ponente de Boheringher Ingelheim. La Dra. Florencia Aranguren recibió honorarios de AstraZeneca, Boehringer Ingelheim, Servier, Sanofi y Temis Lostaló. La Dra. María Cristina Faingold recibió honorarios como disertante de Novo Nordisk, Sanofi, AstraZeneca, y apoyo para una investigación de Sanofi y Novo Nordisk. La Dra. Graciela Fuente recibió honorarios como ponente de AstraZeneca, Craveri y Eli Lilly, y por su participación como consejera para MSD, Novo Nordisk y Sanofi, sin relación de dependencia. El Dr. Mariano Aníbal Giorgi recibió honorarios como ponente de AstraZeneca, Novo Nordisk, y como advisory board de Raffo. El Dr. Juan Nogueira recibió apoyo y financiación para una investigación del CONICET. El Dr. Hugo Sanabria recibió honorarios como ponente y advisory board para AstraZeneca, Boehringer Ingelheim, Eli Lilly y Novo Nordisk, y como ponente para Montpellier, Roemmers y Servier, y financiación para investigación de Bayer y Novo Nordisk. El Dr. Jorge Thierer recibió honorarios como ponente de AstraZeneca, Boehringer Ingelheim y Novartis. El Dr. Isaac Sinay recibió honorarios como ponente de Sanofi Aventis, Novo Nordisk, Boheringher Ingelheim, AstraZeneca y Montpellier. El Dr. Carlos Buso recibió honorarios de Montpellier y Francelab. La Dra. Carla Musso recibió honorarios como ponente de AstraZeneca, Eli Lilly, Sanofi, MSD, Roemmers y Gador, y como advisory board de Abbot, Sanofi, MSD, AMRyT y AstraZeneca. El Dr. Claudio Majul recibió apoyo para investigación clínica de Novo Nordisk, Sanofi, AstraZeneca, Novartis, Eli Lilly, MSD, Takeda y Amgen. Los demás autores no poseen conflictos de interés.

\section{Epidemiología e impacto sanitario}

\section{a. Epidemiología de la diabetes mellitus}

Resulta imperativo comenzar este documento resaltando que la diabetes mellitus (DM) es una de las enfermedades crónicas no transmisibles que tiene más impacto mundial. También resulta apropiado remarcar que intentaremos brindar información epidemiológica local, en caso que esté disponible. En relación a la prevalencia, en Argentina, según lo publicado recientemente en la Cuarta Encuesta Nacional de Factores de Riesgo', el valor registrado para DM o elevación de la glucemia (por autoinforme) fue del $12,7 \%$ (IC 95\% de 21,1 a 13,4) en el año 2018. Resulta muy inquietante que, en términos relativos, la prevalencia de DM o elevación de glucemia se incrementó un 34\% desde 2005. Esta tendencia podría asociarse, entre tantos otros factores, al incremento en la prevalencia de sobrepeso y obesidad (49 y $61,6 \%$ en 2005 y 2018 , respectivamente) y la baja ac- 
tividad física $(54,9$ y 64,9\% en 2009 y 2018, respectivamente). En el caso específico de la DM, hace una década el estudio Cardiovascular Risk Factor Multiple Evaluation in Latin America (CARMELA) informó una prevalencia del 6,2\% (para la Ciudad Autónoma de Buenos Aires) $)^{2}$ y, más recientemente, el estudio Centro de Excelencia en Salud Cardiovascular para el Cono Sur (CESCAS) refiere una prevalencia del 8,4\% (en la Ciudad de San Carlos de Bariloche) ${ }^{3}$.

La DM impacta en términos de mortalidad y morbilidad. En relación con la primera, según la Secretaría de Salud de la Nación, el 2,6\% de todas las defunciones en Argentina fue causada por DM (para ambos sexos y todos los grupos etarios) $)^{4}$. Valores similares $(2,81 \%)$ informa el Global Burden of Disease Study (GBD) 5 . La carga de enfermedad y discapacidad de la DM representa unos 946,61 años de vida ajustados a discapacidad (DALYs, sus siglas en inglés) por cada 100.000 habitantes en $2017^{5}$ y su impacto se ha incrementado a lo largo de los años. De los dos tipos principales de DM, la DM2 representa la mayor carga de enfermedad en términos de prevalencia y morbimortalidad 6 . La prevalencia estimada, por el GBD, de DM2 en Argentina es de $7,44 \%$ (comparada con $0,37 \%$ de la DM1). Del total de muertes en 2017, la DM2 contribuyó con un $2,35 \%(0,46 \%$ para la DM1) y la carga de discapacidad fue de 828,36 DALYs por cada 100.000 habitantes $(108,34$ para la DM1). La carga de enfermedad depende, entre muchos otros determinantes, de la tasa de diagnóstico, el acceso al tratamiento y el control de la enfermedad. Se estima que una de cada cuatro personas con DM desconoce la presencia de la enfermedad. De las que tienen diagnóstico confirmado, aproximadamente tres de cada cuatro personas con DM reciben tratamiento con fármacos, y sólo la mitad de las tratadas tiene niveles de glucemia controlados. En consecuencia, más de la mitad de los pacientes con DM no recibe los cuidados indicados y esto impacta en la probabilidad de sufrir complicaciones ${ }^{3}$.

\section{b. Epidemiología de las complicaciones cardiovasculares en el paciente con DM}

La enfermedad cardiovascular (ECV) es la principal causa de morbimortalidad en los pacientes con $\mathrm{DM}^{7}$. Se estima que la prevalencia de ECV supera el $30 \%$ en los pacientes con DM y que las formas más prevalentes son la enfermedad coronaria y la insuficiencia cardíaca ${ }^{8}$. Pese a que en la última década los cuidados de salud que reciben los pacientes con DM han mejorado, la incidencia de muerte por causa cardiovascular y enfermedad coronaria es más del doble que en pacientes sin DM; además presentan un mayor riesgo de sufrir internaciones por enfermedades cardiovasculares (por ejemplo, síndrome coronario agudo, insuficiencia cardíaca) ${ }^{9}$. Aunque el riesgo de internación es superior en los pacientes con DM1, la mayor carga para los sistemas de salud está representada por los pacientes con DM2 dadas la mayor prevalencia y la mayor presencia de comorbilidades. En Argentina, según estimaciones del GBD, la letalidad de la DM, en especial de la DM2, se ha reducido entre 2007 y 2017. Esto ha tenido como consecuencia un incremento del $11 \%$ en los años de vida con discapacidad (YLD, sus siglas en inglés) para ambos tipos de DM', especialmente en los sujetos con $\mathrm{DM}^{6}$.

El impacto, en cuanto a la utilización de recursos y sus costos asociados, representa una importante carga para el sistema de salud, en especial cuando la complicación es cardiovascular. Tomando como referencia el cuidado recibido por la población sin DM, el costo médico directo del cuidado de los pacientes con DM en Argentina se incrementa 2,4 veces en pacientes con DM sin complicaciones y 3,9 veces cuando desarrollan alguna complicación ${ }^{10}$. Se estima que, en nuestro país, el costo de la hospitalización por causa cardiovascular en DM (que representa más del $40 \%$ de todas las hospitalizaciones en los diabéticos) alcanza más de seis veces el costo incurrido por otras causas ${ }^{11}$.

\section{c. Implicaciones sanitarias}

La información presentada previamente ilustra la carga de enfermedad que enfrentamos en nuestro país a causa de la DM. En este sentido, el proceso de concientización sobre el problema, liderado por la Sociedad Argentina de Diabetes y otras organizaciones no gubernamentales, ha dado como resultado que el Estado aborde este problema de modo más integral. La sanción de la Ley 23.753 (modificada en 2018) brinda un marco de referencia (más allá de los cuestionamientos que naturalmente surgen a todas las normas legales, que son perfectibles) para todos los actores involucrados.

Las exigencias financieras que afrontan los diferentes actores integrantes del muy fragmentado sector de salud de nuestro país hacen que muchos de los recursos diagnósticos y terapéu- 
ticos de reconocido valor científico estén más allá de la capacidad de pago del sistema. Es decir, no siempre estarán disponibles o serán accesibles para todos los pacientes. Esto exige que aquellos que estamos involucrados en el cuidado de los pacientes utilicemos con la mayor racionalidad posible los recursos disponibles. La diversidad de los escenarios asistenciales (público, privado, Seguridad Social) en los que los médicos nos desempeñamos implica que no necesariamente exista disponibilidad o accesibilidad a los recursos que se recomiendan en este documento y su uso estará condicionado, por lo tanto, a la realidad del medio donde se efectúa la atención. Ejemplo de esto es el uso del ultrasonido para la pesquisa de ateromatosis subclínica para estratificar el riesgo en los pacientes con DM. El valor (utilidad en el sentido de capacidad de brindar un beneficio clínico) está reconocido en la literatura médica; sin embargo, el recurso no siempre está disponible y, por lo tanto, se deberá estratificar el riesgo con otras herramientas, también de gran valor pronóstico.

Uno de los temas más complejos, desde el punto de vista de la provisión de servicios de salud a los pacientes con DM, es el grado de cobertura que se les otorga a los medicamentos innovadores (por ejemplo, los inhibidores del cotransportador sodio-glucosa tipo 2 -inhibidores SGLT-2-, o los antagonistas del péptido símil glucagón tipo 1 -antagonistas (LP-1-) o nuevos desarrollos de fármacos ya utilizados (análogos de insulinas, por ejemplo, lispro, glargina, degludec). Muchos de estos fármacos, que en los últimos años demostraron tener un perfil de eficacia y seguridad relevantes (es decir, agregan valor) pues reducen eventos cardiovasculares y renales, todavía pueden no estar accesibles a todos los pacientes. Es por ello que, ante una indicación precisa, si bien la primera intención del tratamiento debería orientarse a utilizar un determinado medicamento, es posible que no exista acceso a él en una proporción importante de pacientes, por lo cual se deberá elegir otra opción terapéutica.

En este sentido, el desarrollo de evaluaciones de economía de la salud locales contribuirá sustancialmente a definir el precio de los recursos en función del valor que aportan a los pacientes.

En conclusión, la epidemia de DM está instalada y sus consecuencias cardiovasculares, en términos de muerte, enfermedad y discapacidad, son alarmantes. Es tarea de todos los involucrados afrontar sistemáticamente la evaluación y el tratamiento de los pacientes con DM para reducir esta carga de enfermedad.

\section{BIBLIOGRAFÍA}

1. Cuarta Encuesta Nacional de Factores de Riesgo. Argentina: Ministerio de Salud; 2019. Disponible en: http://www.msal.gob. ar/images/stories/bes/graficos/0000001622cnt-2019-10_4taencuesta-nacional-factores-riesgo.pdf.

2. Escobedo J, Buitrón LV, Velasco MF, Ramírez JC, Hernández R, Macchia A, et al. High prevalence of DM and impaired fasting glucose in urban Latin America: the CARMELA Study. Diabet Med 2009; 9:864-71.

3. Irazola V, Rubinstein A, Bazzano L, Calandrelli M, Chung-Shiuan C, Elorriaga N, et al. Prevalence, awareness, treatment and control of DM and impaired fasting glucose in the Southern Cone of Latin America. PLoS ONE 2017;12(9): e0183953.

4. Anuario 2017. Ministerio de Salud Argentino: Dirección de Estadísticas e Información en Salud; 2017. Disponible en: http:// www. deis.msal.gov.ar/index.php/anuario-2017.

5. Global Burden of Disease Study. Institute for Health Metrics and Evaluations. Washington: University of Washington; 2017. Disponible en: http://ghdx.healthdata.org/gbd-results-tool.

6. González L, Caporale JE, Elgart JF, Gagliardino JJ. The Burden of DM in Argentina. Glob J Health Sci 2015; 7(3):124-133.

7. Paneni F, Beckman JA, Creager MA, Cosentino F. DM and vascular disease: pathophysiology, clinical consequences, and medical therapy: part I. Eur Heart J 2013; 34:2436-2443.

8. EinarsonTR, Acs A, Ludwig C, Panton UH. Prevalence of cardiovascular disease in type 2 DM: a systematic literature review of scientific evidence from across the world in 2007-2017. Cardiovasc Diabetol 2018; 17(83):1-19.

9. Rawshani A, Rawshani A, Franzén S, Eliasson B, Svensson AM, Miftaraj M, et al. Mortality and cardiovascular disease in type 1 and type 2 DM. N Engl J Med 2017; 376(15):1407-1418.

10. Caporale JE, Elgart JF, Gagliardino JJ. Diabetes in Argentina: cost and management of diabetes and its complications and challenges for health policy. Globalization and Health 2013; 9:54.

11. Caporale JE, Calvo H, Gagliardino JJ. Costos de atención en personas con diabetes previos y posteriores a su hospitalización. Rev Panam Salud Publ 2006; 20:361-368.

\section{Definiciones}

\section{a. Definición de DM}

Los criterios actualmente aceptados para el diagnóstico de DM es la presencia de uno de los tres siguientes ${ }^{1}$ :

- Presencia de síntomas característicos de DM (poliuria, polidipsia y pérdida de peso) junto con una glucemia plasmática aislada (en cualquier momento del día y sin considerar la última ingesta) $\geq 200 \mathrm{mg} / \mathrm{dL}$.

- Glucemia plasmática en ayunas $\geq 126 \mathrm{mg} / \mathrm{dL}$ (posterior a un ayuno de 8 horas -h-).

- Glucemia plasmática obtenida a las $2 \mathrm{~h}$ de la ingestión de $75 \mathrm{~g}$ de glucosa anhidra disuelta en $375 \mathrm{~cm}^{3}$ de agua en el contexto de una prueba de tolerancia oral a la glucosa $\geq 200 \mathrm{mg} / \mathrm{dL}$. 
En ausencia de hiperglucemia franca, estos criterios deben confirmarse nuevamente repitiendo alguna de las determinaciones.

La hemoglobina glicosilada, corrientemente utilizada para guiar el manejo y ajustar el tratamiento, no es un criterio que la SAD aconseje para el diagnóstico debido a la falta de estandarización de la medición en muchos laboratorios en Argentina. Otras sociedades internacionales proponen un valor $\geq 6,5 \%$ como criterio diagnóstico.

\section{b. Definición de glucosa alterada en ayunas y tolerancia alterada a la glucosa}

Existen individuos que presentan glucemias por encima de los valores normales pero que no llegan a los niveles sugeridos para el diagnóstico de DM. Dentro de esa categoría se diagnostica como glucosa alterada en ayunas cuando las glucemias de ayuno se encuentran entre $110 \mathrm{mg} / \mathrm{dL}$ y $125 \mathrm{mg} / \mathrm{dL}$, y tolerancia alterada a la glucosa en aquellos individuos que luego de la sobrecarga con $75 \mathrm{~g}$ de glucosa anhidra en $375 \mathrm{~mL}$ de agua evidencian glucemia basal menor de $126 \mathrm{mg} / \mathrm{dL}$ pero a las $2 \mathrm{~h}$ éstas se encuentran entre $140 \mathrm{mg} / \mathrm{dL}$ y $199 \mathrm{mg} / \mathrm{dL}^{1}$.

\begin{tabular}{|l|c|c|c|}
\hline & Normal & GAA O TAG & DM \\
\hline GPA $(\mathrm{mg} / \mathrm{g}$ & $<110 \mathrm{mg} / \mathrm{dL}$ & Entre $110-126$ & $\geq 126$ \\
\hline $2 \mathrm{~h}$ post carga $(\mathrm{mg} / \mathrm{dL})$ & $<140 \mathrm{mg} / \mathrm{dL}$ & Entre $140-199$ & $\geq 200$ \\
\hline
\end{tabular}

GAA: glucosa alterada en ayunas; TAG: tolerancia alterada a la glucosa; GPA: glucemia plasmática en ayunas.

Tabla 1: Criterios para diagnóstico de glucosa alterada en ayunas y tolerancia alterada a la glucosa.

Tanto la glucosa alterada en ayunas (GAA) como la tolerancia alterada a la glucosa (TAG) son, por un lado, factores de riesgo para el desarrollo de ECV $y$, por otro, conllevan un riesgo aumentado de desarrollo de DM (4\% por año en GAA y $9 \%$ por año en TAG). Las personas que presentan glucemias entre $100 \mathrm{mg} / \mathrm{dL}$ y $110 \mathrm{mg} / \mathrm{dL}$ sumadas a factores de riesgo para el desarrollo de DM pueden también requerir controles más cercanos.

El diagnóstico de DM, GAA y TAG se efectuará siguiendo los criterios diagnósticos establecidos por la SAD. Grado de recomendación: I. Nivel de evidencia: B.

\section{c. Pesquisa de DM}

Dado que entre un cuarto y la mitad de las personas con DM permanecen sin diagnóstico, debe tenerse en cuenta una serie de factores de riesgo asociados al desarrollo de DM, ante cuya presencia debe efectuarse la pesquisa. Esta búsqueda se fundamenta en el hecho de que, cuanto más temprano se hace el diagnóstico de DM, tanto mejor es su pronóstico y más fácil su tratamiento². Debería considerarse en toda persona adulta con sobrepeso (índice de masa corporal -IMC- igual a $25 / \mathrm{m}^{2}$ o mayor) y que tenga uno o más factores de riesgo adicionales para presentar DM como:

- Sedentarismo.

- Familiares de primer grado con DM.

- Etnias con mayor riesgo.

- Antecedentes de DM gestacional o recién nacidos con peso mayor de $4 \mathrm{~kg}$.

- Hipertensión arterial $(\geq 140 / 90 \mathrm{mmHg}$ o con tratamiento establecido).

- HDL colesterol menor de 35 mg/dL y/o triglicéridos mayores de $250 \mathrm{mg} / \mathrm{dL}$.

- Diagnóstico de síndrome de ovario poliquístico (mujeres).

- Hemoglobina glicosilada (HbA1c) $\geq 5,7 \%$, TAG - GAA en estudios anteriores.

- Otras condiciones clínicas asociadas a insulinorresistencia (obesidad grave, acantosis nigricans).

- Antecedentes de enfermedad cardiovascular.

En el resto de la población, la pesquisa de DM debería comenzar luego de los 45 años de edad. Si los resultados son normales, debería repetirse cada tres años, pero puede acortarse el período de tiempo según los resultados iniciales y el nivel de riesgo. Los estudios diagnósticos deberían ser la glucemia en ayunas o la prueba de tolerancia oral a la glucosa (PTOG) con $75 \mathrm{~g}^{3,4}$.

Cabe remarcar que la presencia de enfermedad cardiovascular, como por ejemplo el antecedente de internación por síndrome coronario agudo como insuficiencia cardíaca, se asocia a un mayor riesgo de desarrollar DM, el cual empeora el pronóstico, por lo que debe estarse atento a la pesquisa rutinaria de DM. Por otra parte, la presencia de síndrome metabólico, o bien la de algunos de sus componentes, genera un riesgo aumentado tanto de ECV como de DM.

La pesquisa de DM se efectuará en todo adulto mayor con sobrepeso u obesidad y factores de riesgo asociados o ante la presencia de ECV, utilizando glucosa plasmática en ayunas o PTOG. Grado de recomendación: I. Nivel de evidencia: B. 


\section{d. Tipos de DM}

Es importante conocer los distintos tipos de DM para clasificarla e implementar el tratamiento adecuado. El diagnóstico temprano es importante a fin de evitar las complicaciones microvasculares y macrovasculares de la enfermedad, estas últimas muy prevalentes y la primera causa de muerte de los pacientes con DM.

- DM1. Representa menos del 10\% del universo de pacientes con DM y está mediada generalmente por la presencia de autoanticuerpos, lo que deriva en la destrucción progresiva y rápida de la masa celular beta. Los marcadores de este proceso incluyen autoanticuerpos contra las células del islote (ICA, sus siglas en inglés), autoanticuerpos contra el ácido glutámico descarboxilasa (GADA), contra la insulina (AAl) y los anticuerpos antiirosina fosfatasa (IA2). Estos pacientes tienen predisposición a padecer otras enfermedades autoinmunes como enfermedad de Graves, hipotiroidismo, enfermedad celíaca, enfermedad de Addison, anemia perniciosa y vitiligo. Se asocia a polimorfismos del antígeno leucocitario humano (HLA, sus siglas en inglés). Ocurre comúnmente en la infancia y adolescencia, aunque puede presentarse a cualquier edad. Presenta bajas concentraciones de péptido $\mathrm{C}$. Algunas formas de DM1 no tienen etiología conocida, por lo que se consideran idiopáticas, y también se presentan con insulinopenia con tendencia a la cetosis pero sin evidencia de autoinmunidad. En adultos puede aparecer una forma clínica de instalación más lenta denominada LADA (en inglés, latent auto-immune DM in adults) con grado diverso de insulinopenia.

- DM2. Esta presentación clínica, que abarca aproximadamente al $90 \%$ de los pacientes con DM, implica una disminución en la sensibilidad periférica y hepática a la insulina, y un déficit relativo en su secreción en relación con el grado de insulinorresistencia. Es una enfermedad poligénica y se asocia con predisposición genética. Desde el punto de vista fisiopatológico muestra mayor diversidad de mecanismos, que van desde la insulinorresistencia con déficit de acción de insulina hasta la insulinopenia agravada por la disminución de la sensibilidad periférica. Otros mecanismos involucrados son: un menor efecto incretina, un aumento en la secreción de glucagón, una mayor reabsorción de glucosa a nivel del túbulo proximal renal, alteraciones a nivel de neurotransmisores, etc. La obesidad, y en especial la abdominal, se presenta en un porcentaje muy elevado de pacientes. Suele asociarse con dislipidemia e hipertensión arterial. El riesgo de padecer DM2 aumenta con la edad, obesidad, sedentarismo, DM gestacional previa y aspectos epigenéticos. Hoy en día se consideran también como factores predisponentes los cambios en el microbioma.

- DM gestacional. Definida como glucemia alterada en ayunas o tolerancia alterada a la glucosa con comienzo durante el embarazo. El diagnóstico se efectúa ante la presencia de glucemia en ayunas entre 100 y $125 \mathrm{mg} / \mathrm{dL}$ en dos días diferentes (durante la misma semana) y/o cuando la glucemia a las $2 \mathrm{~h}$ postsobrecarga con $75 \mathrm{~g}$ de glucosa anhidra es $\geq 140 \mathrm{mg} / \mathrm{dL}$, determinación que se sugiere efectuar entre las semanas 24 y 28 de gestación. Si bien esta condición revierte en la mayoría de los casos al momento del parto, el antecedente de DG constituye una subpoblación de mujeres con alto riesgo de desarrollar DM posteriormente. Entre un 5 y un 10\% de estas pacientes pueden desarrollar DM en el período posparto y alrededor de un $50 \%$ durante los 10 años siguientes.

- Otros tipos específicos de DM. Dentro de este grupo, que abarca $<3 \%$ de los pacientes con DM, deben considerarse ocho categorías diferentes: defectos genéticos de la célula beta, defectos genéticos de la acción de la insulina, enfermedades del páncreas exocrino, endocrinopatías, DM inducida por fármacos, DM inducida por infecciones, formas poco comunes inmunomediadas y síndromes genéticos asociados. 
Revista de la Sociedad Argentina de Diabetes Año 55 Vol. 55 № 1 Suplemento Consenso Sociedad Argentina de CardiologíaSociedad Argentina de Diabetes Enero-abril de 2021: 03-70 ISSN 0325-5247 (impresa) ISSN 2346-9420 (en línea)

\begin{tabular}{|c|c|c|}
\hline Evaluación & Prueba & Frecuencia \\
\hline Evaluación bucal & Gingivitis, estado de piezas dentarias & Anual \\
\hline Evaluación cardiovascular & $\begin{array}{l}\text { Examen físico cardiovascular y electrocardiograma } \\
\text { (otras evaluaciones. Ver capítulo: Estratificación de riesgo) }\end{array}$ & $\mathrm{Al}$ menos anualmente \\
\hline Evaluación hepática & $\begin{array}{l}\text { Ecografía hepática (evaluar presencia de hígado graso) } \\
\text { Hepatograma (ver Laboratorio) }\end{array}$ & $\mathrm{Al}$ menos una vez \\
\hline Evaluación oftalmológica & Agudeza visual, examen de retina con dilatación de pupilas & Anual*** \\
\hline Inmunización & $\begin{array}{l}\text { Influenza } \\
\text { Vacuna antineumocócica: } \\
\text {. Vacuna conjugada antineumocócica de } 13 \text { serotipos (VCN13) } \\
\text {. Vacuna polisacárida antineumocócica de } 23 \text { serotipos (VPN23) } \\
\text { Hepatitis B } \\
\text { Doble bacteriana }\end{array}$ & $\begin{array}{l}\text { Anual } \\
1^{\circ} \text { dosis: VCN13 } \\
2^{\circ} \text { dosis: VPN23 (intervalo mínimo de } 12 \text { meses luego } \\
\text { de la VCN13) } \\
1^{\circ} \text { refuerzo: VPN23 a los } 65 \text { años (respetando intervalo } \\
\text { mínimo de } 5 \text { años de la dosis anterior) } \\
\text { Única vez (con esquema completo) } \\
\text { Cada } 10 \text { años }\end{array}$ \\
\hline Laboratorio & $\begin{array}{l}\text { Función renal: } \\
\text {. Estimación de filtrado glomerular por fórmula } \\
\text {. Relación albuminuria-creatininuria en orina espontánea o } \\
\text { proteinuria en orina } 24 \text { h: } \\
\text { Glucemia: } \\
\text {. Hemoglobina glicosilada A1c } \\
\text {. Hepatograma } \\
\text { Perfil lipídico: } \\
\text { Colesterol total, LDL colesterol, HDL colesterol y triglicéridos. } \\
\text { Estimación de colesterol no HDL }\end{array}$ & $\begin{array}{l}\text { Cada } 3 \text { a } 6 \text { meses } \\
\text { Cada } 3 \text { a } 6 \text { meses } \\
\text { Anual } \\
\text { Al menos anual }\end{array}$ \\
\hline Peso/IMC*/CC** & & En cada visita \\
\hline Pies & $\begin{array}{l}\text { Examen físico: } \\
\text {. Evaluar neuropatía: reflejos osteotendinosos, sensibilidad vibratoria } \\
\text { (diapasón } 256 \mathrm{~Hz} \text { ) y táctil profunda (monofilamento). Signo del } \\
\text { abanico (hipotrofia muscular) } \\
\text { Evaluación vascular: } \\
\text {. Presencia de pulsos pedios y tibiales, temperatura de las extremidades } \\
\text { Evaluar alteraciones ortopédicas (dedos en garra, hallux valgus) } \\
\text {. Otras evaluaciones: integridad de la piel, presencia de hiper- } \\
\text { queratosis, presencia de vello, presencia de micosis interdigital y } \\
\text { onicomicosis }\end{array}$ & $\begin{array}{l}\text { Inspección visual: cada visita } \\
\text { Sin lesiones y pie de bajo riesgo: anual } \\
\text { Pie de alto riesgo y con lesión: } \\
\text { determinar por especialista }\end{array}$ \\
\hline Presión arterial & Sentado, tres veces con intervalos y obteniendo promedios & En cada visita \\
\hline
\end{tabular}

*IMC: índice de masa corporal; **CC: circunferencia de cintura; ***Si fuera normal, podría realizarse cada dos años.

Tabla 2: Seguimiento del paciente con DM.

\section{BIBLIOGRAFÍA}

1. Gagliardino JJ, Sereday M, González C, Domínguez JM, Mazza CM. Consenso sobre criterio diagnóstico de la glucemia de ayunas alterada de la Sociedad Argentina de Diabetes. Revista de la Sociedad Argentina de Diabetes 2007; 41(3):95-104.

2. World Health Organization. Géneva. 2003. Screening for type 2 diabetes. Report of a World Health Organization and International DM Federation meeting. Disponible en: https://apps.who. int/iris/handle/10665/68614.
3. World Health Organization. Genova: WHO Department of Non communicable Disease Management; 2003. Acceso: 18 de julio de 2019. Screening for type 2 diabetes. Report of a World Health Organization and International Diabetes Federation meeting. Disponible en: https://www.who.int/diabetes/publications/en/ screening_mnc03.pdf

4. American Diabetes Association. Standards of Medical Care in Diabetes. Classification and diagnosis of diabetes mellitus. Diabetes Care 2019; 42 (Supp 1): S13-S28.

\section{Estratificación de riesgo}

\section{a. Estratificación de riesgo cardiovascular en el paciente con DM}

Los sistemas de estratificación de riesgo son herramientas para organizar el uso de recursos destinados a brindar servicios en prevención car- diovascular. No son instrumentos de precisión; en consecuencia, es deseable que su uso sea una aproximación a la evaluación de riesgo y no deben considerarse una "verdad tallada en piedra". La SAC y la SAD han respaldado, en 2009, la Guía de Prevención de Enfermedades Cardiovasculares del Ministerio de Salud de la Nación (MSAL)ํ. Esta 
guía, desarrollada por la Organización Mundial de la Salud (OMS) en 2007 propone un instrumento de estimación del riesgo cardiovascular a 10 años (Tabla 1) mediante un sistema de puntajes basado en el sexo, la edad, los valores de colesterol total y la tensión arterial sistólica y el tabaquismo². Debido a que el riesgo cardiovascular en los diabéticos es, en general, mayor que en los no diabéticos, el sistema de estimación de riesgo del Ministerio propone el uso de una tabla específica para diabéticos (independientemente del tipo de DM). Una de las principales fortalezas de este instrumento reside en que está desarrollado con datos epidemiológicos provistos por los estados miembros de la OMS. Para Argentina, la tabla de riesgo disponible corresponde a la Región B de las Américas y permite estimar el riesgo de desarrollar eventos cardiovasculares fatales y no fatales en esta subregión dado que, hasta el momento, es el único sistema de estimación de riesgo que cuenta con datos locales/regionales ${ }^{3}$. La estratificación de riesgo permite definir grupos de pacientes más vulnerables y, por lo tanto, asignarles recursos terapéuticos de mayor eficacia. Una de las principales características de las categorías de riesgo del sistema OMS-MSAL es que basa las categorías de riesgo y los recursos terapéuticos no sólo en la morbimortalidad esperada sino en aspectos relacionados con la estructura, funcionamiento y financiamiento de los sistemas de salud ${ }^{2}$. Esto permite, según el grado de disponibilidad de recursos, definir estrategias de prevención. En consecuencia, uno de los principales puntos cuestionados por muchos expertos es que este sistema subestima el riesgo cardiovascular, en especial en los diabéticos. El criterio adoptado se basa en las categorías de ingreso per cápita, que considera que el umbral de riesgo para las terapéuticas más intensivas debe ser un riesgo a 10 años $\geq 30 \%$ en países como Argentina. Se incluyen en este grupo la enfermedad cardiovascular establecida y la DM con nefropatía (u otra enfermedad renal). Si bien se sabe que los diabéticos (en especial aquellos con DM de menos de 10 años de evolución) no tienen el mismo riesgo cardiovascular que los pacientes con enfermedad coronaria ${ }^{4,5}$, los criterios del sistema aplicado en Argentina se contradicen con la opinión de muchas otras sociedades científicas. Por ejemplo, el puntaje (score) para países europeos de alto riesgo, versión 2016 (de la European Society of Cardiology, ESC) 6 o el calculador de riesgo de 2013 ACC/AHA Guideline for Assessment of Cardiovascular Risk (desarrollado en los Estados Unidos conjuntamente por el American College of Cardiologist y la American Heart Association) ${ }^{7}$ estratifican a los pacientes con DM como una población de riesgo intermedio, alto o muy alto. De hecho, las últimas Guías de la ESC y la European Association for the Study of Diabetes (EASD) refrendan esta posición.

\section{Recomendaciones}

- El abordaje inicial del riesgo CV debe basarse en la tabla de predicción de la región $B$ de las Américas de la OMS (Grado de recomendación I. Nivel de evidencia B. Tabla 1).

- Se consideran de alto riesgo (equivalente a categoría de 20 a $30 \%$ de la tabla) los pacientes con DM de $\geq 10$ años de evolución con algún otro factor de riesgo asociado y sin daño de órgano blanco (Grado de recomendación I. Nivel de evidencia C).

- Se consideran de muy alto riesgo (equivalente a categoría de $\geq 30 \%$ de la tabla) los pacientes con DM con: 1) enfermedad cardiovascular previa (evento sintomático o asintomático coronario, cerebrovascular o vascular periférico en cualquier territorio); 2) ateromatosis subclínica asociada (presencia de placas en algún territorio vascular, score de calcio coronario elevado, índice de tobillo brazo $<0,9)$; 3) daño de órgano blanco (proteinuria, aclaramiento -clearance de creatinina de $\leq 30 \mathrm{~mL} / \mathrm{min}$, retinopatía, hipertrofia ventricular izquierda o lesiones tróficas en miembros inferiores) (Grado de recomendación I. Nivel de evidencia C).

- En pacientes con alto o muy alto riesgo no es preciso utilizar la tabla de estratificación (Grado de recomendación I. Nivel de evidencia C).

- En pacientes con riesgo bajo e intermedio por tabla (categorías de menos de 20\%) podría ser de utilidad pesquisar ateromatosis subclínica, si estuviera disponible (Grado de recomendación I. Nivel de evidencia C).

- En pacientes con DM sin evento cardiovascular previo se sugiere una evaluación cardiovascular, al menos una vez al año y, de ser posible, realizada por un médico especialista en Cardiología (Grado de recomendación I. Nivel de evidencia C). 


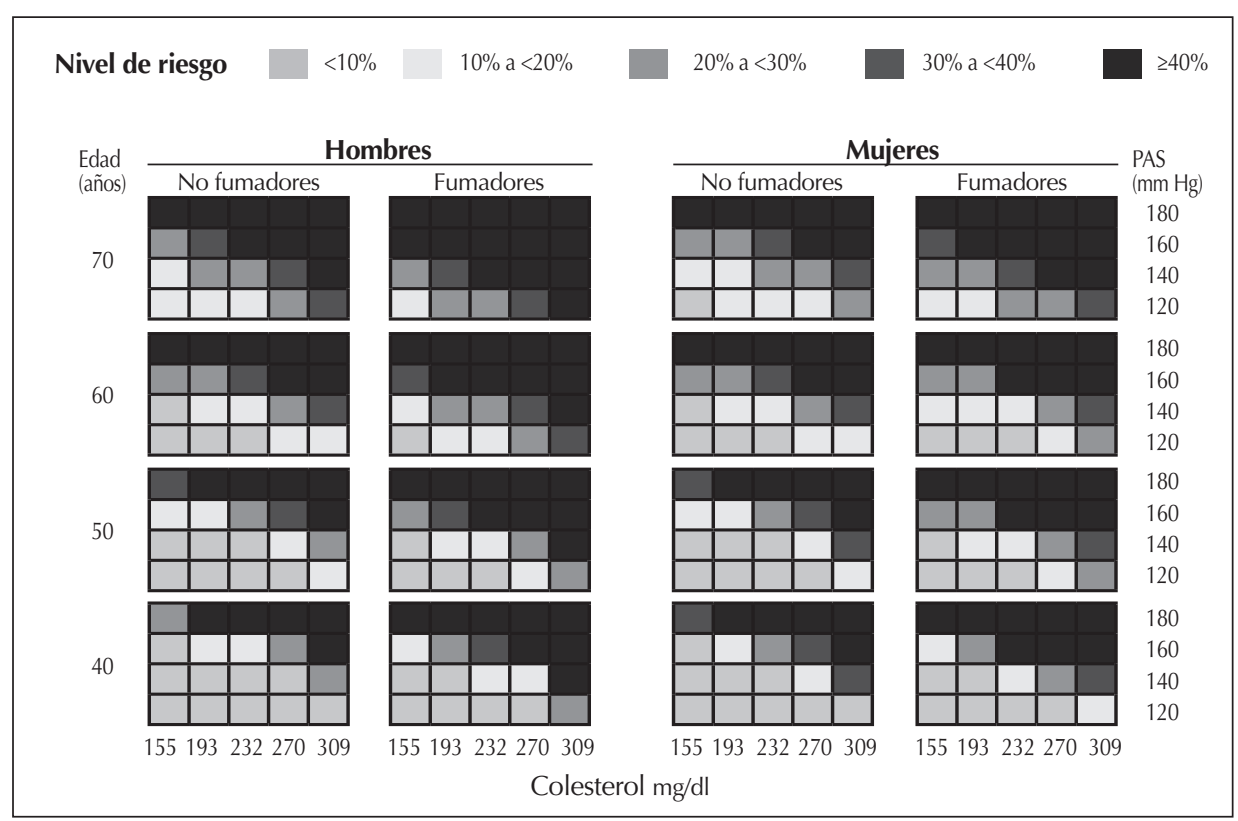

Tabla 1: Tabla de predicción de la región B de las Américas de la OMS.

\section{b. Pesquisa de isquemia miocárdica silente}

La detección de isquemia silente constituye un desafío de la Cardiología y, más aún, cuando se trata de pacientes con DM. La población con DM presenta entre dos y cuatro veces más riesgo de desarrollar enfermedad cardiovascular al tiempo que la tasa de eventos asintomáticos o con síntomas atípicos resulta mayor que en pacientes sin la comorbilidad ${ }^{9}$. La isquemia silente afecta a un $20-35 \%$ de esta población y representa un factor de riesgo sumamente importante relacionado con alteraciones en la fisiología endotelial y de la microcirculación ${ }^{10}$.

La lógica que se percibe es que, dado que la DM es una enfermedad que promueve la instalación acelerada de aterosclerosis, encontrar aquellos pacientes con isquemia para tratarlos tempranamente podría mejorar el pronóstico futuro y prolongar su sobrevida. Sin embargo, los resultados de la evidencia que disponemos contradicen esta lógica. El estudio Detection of Ischemia in Asymptomatic Diabetics (DIAD) fue diseñado para este propósito con una cohorte de 1.123 pacientes y no pudo encontrar beneficios ${ }^{11}$. En este sentido, un metaanálisis posterior que involucró 3.315 pacientes de cinco estudios, incluido el DIAD, tampoco logró evidenciar beneficio ${ }^{12}$, razón por la cual la American Diabetes Association (ADA) no recomienda el tamizaje rutinario de isquemia en pacientes con $\mathrm{DM}^{13}$.

A partir del trabajo de Haffner et al. ${ }^{14}$, se igualó el riesgo de los pacientes con DM al propio de quienes tuvieron un evento $C V$, estableciéndose el paradigma de la equidad entre ambas condiciones. Pero dicha creencia fue superada y hoy sabemos que no es así sino que, por más de que la DM acelere la progresión de placa ${ }^{15}$, los pacientes con DM constituyen un mosaico heterogéneo de riesgo. Es entonces, en este contexto, donde proliferan las preguntas en torno al tema que estamos tratando: ¿es necesario pesquisar la presencia de isquemia en todos los pacientes con DM? ¿Las pruebas evocadoras de isquemia alcanzan por sí mismas o es preciso sensibilizar previamente la búsqueda con una valoración de riesgo? ¿El riesgo debe medirse con puntajes de riesgo, con estudios complementarios o con ambos?

Si bien no se puede recomendar la pesquisa asintomática de isquemia en este tipo de pacientes, es posible que exista algún nicho de pacientes con DM que se beneficie del tamizaje temprano de enfermedad coronaria subclínica. En este sentido, el estudio DIAD encontró predictores independientes de presencia de isquemia en estudios evocadores y que se relacionan con el sexo masculino, valores de creatinina, disfunción autonómica cardíaca, enfermedad vascular periférica (EVP) y el nivel de C-LDL. Por su parte, el estudio Basel Asymptomatic High-Risk Diabetics Outcome Trial (BARDOT) ${ }^{16}$ también identificó parámetros independientes de riesgo de isquemia silente citando nuevamente el sexo masculino y la 
presencia de EVP, agregando la duración de la DM, el tabaquismo, hipertensión arterial (HTA) y valores de péptido natriurético cerebral elevado (BNP, sus siglas en inglés), agregando algo de valor a la propuesta anterior. En efecto, este trabajo demuestra que la presencia de hasta uno de dichos predictores implica una tasa de anomalía de pruebas evocadoras del $3,2 \%$, cifra que se eleva al $20,7 \%$ en caso de tener dos de ellos y a $47 \%$ si el paciente tuviera tres o más de ellos. Esta circunstancia hace pensar que la detección de isquemia silente desempeña un papel en este tipo de pacientes pero que se necesitarán nuevos estudios para aclarar tal incertidumbre. En consonancia con lo que hemos mencionado, las recomendaciones más recientes dan soporte a la pesquisa de isquemia en pacientes con alto riesgo ${ }^{8,17}$.

\section{Recomendaciones}

- La pesquisa de isquemia silente en pacientes con DM y alto riesgo cardiovascular podría ser de utilidad (Grado de recomendación Ilb. Nivel de evidencia B).

\section{BIBLIOGRAFÍA}

1. Guía de bolsillo para la estimación y el manejo del riesgo cardiovascular. Prevención de las enfermedades cardiovasculares. Ministerio de Salud de la Nación. 2009 Consenso de prevención cardiovascular. Rev Argent Cardiol 2012;80(Supl2):1-127.

2. Prevention of cardiovascular disease: guidelines for assessment and management of total cardiovascular risk. World Health Organization. Disponible en: www.who.int/cardiovascular_diseases/publications/Prevention_of_Cardiovascular_Disease/en/.

3. Carrillo-Larco RM, Altez-Fernández C, Pacheco-Barrios N, Bambs C, Irazola V, Miranda JJ, et al. Cardiovascular disease prognostic models in Latin America and the Caribbean. A systematic review. Glob Heart 2019; 14(1):81-93.

4. Evans JM, Wang J, Morris AD. Comparison of cardiovascular risk between patients with type $2 \mathrm{DM}$ and those who had had a myocardial infarction: cross sectional and cohort studies. BMJ 2002; 324:939-942.

5. Wannamethee SG, Shaper AG, Whincup PH, Lennon L, Sattar N. Impact of DM on cardiovascular disease risk and all-cause mortality in older men: influence of age at onset, DM duration, and established and novel risk factors. Arch Intern Med 2011; 171:404-410.
6. 2016 European Guidelines on cardiovascular disease prevention in clinical practice. The sixth joint task force of the European Society of Cardiology and other societies on cardiovascular disease prevention in clinical practice (constituted by representatives of 10 societies and by invited experts): Developed with the special contribution of the European Association for Cardiovascular Prevention \& Rehabilitation (EACPR). Eur J Prev Cardiol 2016; 23(11):NP1-NP96.

7. Goff DC Jr, Lloyd-Jones DM, Bennett G, Coady S, D’Agostino RB Sr, Gibbons R, Greenland P, Lackland DT, Levy D, O'Donnell CJ, Robinson JG, Schwartz JS, Shero ST, Smith SC Jr, Sorlie P, Stone NJ, Wilson PW; American College of Cardiology/ American Heart Association. Task force on practice guidelines. 2013 ACC/AHA Guideline on the Assessment of Cardiovascular Risk. A Report of the American College of Cardiology/American Heart Association Task Force on Practice Guidelines. J Am Coll Cardiol 2014; 1,63(25 Pt B):2935-59.

8. 2019 ESC Guidelines on diabetes, pre-diabetes, and cardiovascular diseases developed in collaboration with the EASD. Eur Heart J 2020 Jan 7;41(2):255-323.

9. Barthelemy O, Lê Feuvre C, Timsit J. Silent myocardial ischemia screening in patients with diabetes mellitus. Arq Bras Endocrinol Metab 2007; 51(2):285-93.

10. Cosson E, Nguyen MT, Chanu B, Banu I, Chiheb S, Balta C, et al. Cardiovascular risk prediction is improved by adding asymptomatic coronary status to routine risk assessment in type 2 diabetic patients. Diabetes Care 2011; 34(9):2101-7.

11. Wackers FJ, Young LH, Inzucchi SE, Chyun DA, Davey JA, Barret $E J$, et al. Detection of silent myocardial ischemia in asymptomatic diabetic subjects: the DIAD study. Diabetes Care 2004; 27(8):1954-61.

12. Rados DV, Pinto LC, Leitao CB, Gross JL. Screening for coronary artery disease in patients with type 2 DM: a meta-analysis and trial sequencial analysis. BMJ Open 2017; 7:e015089.

13. American Diabetes Association. 10. Cardiovascular disease and risk management: Standards of Medical Care in Diabetes 2019. Diabetes Care 2019; 42(Suppl1):S103-S123.

14. Haffner SM, Lehto S, Rönnemaa T, Pyörälä K, Laakso M. Mortality from coronary heart disease in subjects with type 2 DM and in non-diabetic subjects with and without prior myocardial infarction. N Engl J Med 1998; 339:229-34.

15. Wong ND, Sciammarella MG, Polk D, Gallagher A, MirandaPeats L, Whitcomb B, et al. The metabolic syndrome, DM, and subclinical atherosclerosis assessed by coronary calcium. J Am Coll Cardiol 2003; 41(9):1547-53.

16. Zellweger MJ, Haaf $P$, Maraun M, Osterhues $\mathrm{HH}$, Keller U, MuIler-Brand J, et al. Predictors and prognostic impact of silent coronary artery disease in asymptomatic high-risk patients with diabetes mellitus. Int J Cardiol 2017; 244:37-42.

17. Sociedad Argentina de Cardiología. Consenso de síndromes coronarios crónicos-2019. Disponible en: https://www.sac.org. ar/wp-content/ uploads/2019/11/consenso-csc-2019.pdf.

\section{Tratamiento no farmacológico de la hiperglucemia}

\section{a. Plan alimentario}

La terapia nutricional (TN) se recomienda para todos los pacientes con DM y enfermedad CV, y deberá ser individualizada para lograr los objetivos del tratamiento. Para ello, deben tenerse en cuenta las metas recomendadas por la ADA y las recientes recomendaciones de la Sociedad Española de Arteriosclerosis 2019 '.

La TN en pacientes con DM2 logró descensos del 1 al $2 \%$ en la HbA1c según la duración de la enfermedad ${ }^{2}$. Si bien debe prescribirse individualmente, la recomendación de porciones de alimentos saludables suele ser suficiente. Dado que es frecuente la coexistencia de dislipidemia e hipertensión arterial, la TN debe tener en cuenta los pa- 
Revista de la Sociedad Argentina de Diabetes Año 55 Vol. 55 № 1 Suplemento Consenso Sociedad Argentina de CardiologíaSociedad Argentina de Diabetes Enero-abril de 2021: 03-70 ISSN 0325-5247 (impresa) ISSN 2346-9420 (en línea)

rámetros cardiometabólicos para asegurar el éxito de la intervención.

Múltiples planes alimentarios demostraron reducción del riesgo cardiovascular ${ }^{3}$. Varios estudios prospectivos de cohortes destacan los beneficios de la dieta mediterránea en la prevención de enfermedades cardiovasculares y en la reducción de las tasas de mortalidad cardiovascular. Los estudios European Prospective Investigation into Cancer and Nutrition Study (EPIC) y Healthy Life Expectancy (HALE) han confirmado la asociación epidemiológica entre la adherencia a la dieta mediterránea y una menor mortalidad por cualquier causa. El Lyon Diet Heart Study (LDHS) fue el primer estudio de intervención en concluir que el patrón de dieta mediterránea era una estrategia no farmacológica eficaz en prevención secundaria para disminuir los eventos clínicos coronarios en pacientes que habían sobrevivido a un infarto de miocardio. El estudio PREvención con Dleta MEDiterránea (PREDIMED), realizado en individuos de alto riesgo cardiovascular pero sin antecedentes de enfermedad vascular isquémica, demostró que una dieta de tipo mediterráneo conseguía una reducción del $30 \%$ en la incidencia de enfermedades cardiovasculares.

El valor calórico total (VCT) depende del peso, la talla, el sexo, la edad, la situación biológica y el grado de actividad física del paciente. En DM2, más del $80 \%$ presenta sobrepeso u obesidad, por lo cual en estos casos se recomienda la pérdida de peso para mejorar la insulinorresistencia ${ }^{4}$. En este contexto, las dietas con restricción de grasas se han mostrado eficaces en reducir la enfermedad cardiovascular ${ }^{5,6}$.

El aporte proteico ideal debe representar entre un 10 y $20 \%$ del VCT en pacientes con DM sin enfermedad renal'. Con filtrado normal, el consumo debe ser el normal, es decir, 0,8 a $1 \mathrm{~g}$ por kg de peso teórico, aunque en el curso de una caída del filtrado glomerular deberá reducirse la cantidad de proteínas a no menos de 0,6 g por kg de peso teórico por día.

En materia de hidratos de carbono, no existe evidencia concluyente sobre la cuantificación, por lo que tanto el conteo de hidratos como la estimación por porciones son estrategias válidas para lograr el control glucémico. Los carbohidratos recomendados son los provenientes de vegetales, frutas, granos, legumbres y sus productos. Los hidratos de carbono de bajo índice y carga glucémia exigen menor secreción de insulina y de glucagón, mejorando la insulinorresistencia y la insulinopenia ${ }^{8,9}$.
Los edulcorantes no calóricos y los azúcar-alcoholes son seguros consumidos en las cantidades diarias recomendadas (RDA, sus siglas en inglés). La indicación de fibra dietética y granos enteros en los pacientes con DM es igual que para la población general: se encuentra en el orden de $25 \mathrm{~g} / \mathrm{día}$. El mayor beneficio lo adquiere por enlentecer la absorción de los carbohidratos. La utilización de fructosa presente en la fruta puede mejorar el control glucémico pero, si se utiliza fructosa como endulzante, su consumo puede sugerirse en $0,12 \%$ del VCT. Resulta importante aclarar que debe evitarse el consumo para reducir el riesgo del aumento de peso y empeorar el riesgo cardiometabólico. Los edulcorantes no nutritivos y los edulcorantes hipocalóricos se usan para reducir el total de calorías y carbohidratos. No hay evidencia concluyente sobre la ingesta total de grasas, por lo que las metas deben ser individualizadas, siendo la calidad de las grasas lo más importante a tener en cuenta. En DM2, las dietas mediterráneas, ricas en ácidos grasos monoinsaturados (MUFA, sus siglas en inglés), mejoran los lípidos plasmáticos y los factores de riesgo de enfermedad cardiovascular (Nivel de evidencia B). Los ácidos grasos omega 3 (EPA y DHA) de pescados grasos y omega 3-ácido linolénico (ALA) se recomiendan por su efecto beneficioso sobre las lipoproteínas y la prevención de ECV. Las grasas saturadas, el colesterol dietario y los ácidos grasos trans se recomiendan en igual magnitud que para la población general. Los estanoles y esteroles vegetales pueden reducir el LDL-colesterol con una ingesta diaria de 1,6 a $3 \mathrm{~g}$. Las bebidas alcohólicas no deben indicarse específicamente, pero si el paciente opta por su ingesta, se debe aclarar que su consumo aumenta el riesgo de hipoglucemia, especialmente en quienes reciben secretagogos. La recomendación de ingesta de sodio en DM es la misma que para la población general: debe ser menor de 2.300 mg/día (Grado de recomendacion I. Nivel de evidencia B). En caso de hipertensión arterial concomitante deberá indicarse una reducción adicional personalizada.

\section{Recomendaciones}

- El plan de alimentación del paciente con DM debe ser individualizado, con reducción calórica en pacientes con exceso de peso corporal (Grado de recomendación I. Nivel de evidencia A).

- Se deben indicar hidratos de carbono de absorción lenta, restricción de grasas saturadas, propor- 
ciones adecuadas de grasas monoinsaturadas y poliinsaturadas, y proteínas acorde con la función renal (Grado de recomendación I. Nivel de evidencia B).

\section{b. Actividad física}

El concepto actividad física representa todos los movimientos que incrementan el uso de energía (caminar, subir escaleras, etc.), mientras que con ejercicio se hace referencia a la actividad física estructurada. La incorporación de ambos como parte del cambio de estilo de vida implica una mejoría en el control de la glucemia y de los demás factores de riesgo $\mathrm{CV}$, al tiempo que previene el desarrollo de DM en personas con intolerancia oral a la glucosa ${ }^{10}$. Acorde con las recomendaciones de la Sociedad Europea de Cardiología de 20197 se recomienda una actividad física de moderada a vigorosa, en combinación de ejercicio aeróbico y resistencia, $\geq 150$ minutos por semana para la prevención y el control de la DM2. Los programas con intensidad moderada de entrenamiento que utilizan pesos ligeros y con numerosas repeticiones pueden ser útiles para mantener y aumentar la fuerza muscular del tren superior. No obstante, en personas con DM se debe tener precaución si presentan alguna complicación crónica en un estadio avanzado que pueda contraindicar el ejercicio de resistencia como, por ejemplo, HTA no controlada, neuropatía autonómica, retinopatía proliferativa no tratada, historia de lesiones en pies, entre otras 1 .

Es importante destacar que los pacientes tratados con insulina, sulfonilureas o meglitinidas pueden desarrollar hipoglucemia durante el ejercicio o luego de éste por lo cual, en ocasiones, se vuelve necesario un ajuste de la dosis o la suplementación con hidratos de carbono ${ }^{11}$. Por lo tanto, es importante tener presente algunos lineamientos generales, útiles en la regulación de la respuesta de la glucemia a la actividad física (Cuadro 1).

- Monitorear la glucosa sanguínea antes y después del ejercicio (o durante su desarrollo si se considera necesario), especialmente en las primeras sesiones.

- Evitar realizar ejercicio si la glucemia en ayunas es mayor de $250 \mathrm{mg} / \mathrm{dL}$, si se confirma una cetosis presente o si la glucemia es mayor de $300 \mathrm{mg} / \mathrm{dL}$, independientemente de la presencia de cetoacidosis.

- Ingerir carbohidratos antes del ejercicio si la glucemia es $<100$ mg/dL.

- Disponibilidad de carbohidratos durante el ejercicio y al finalizarlo, en especial cuando son de larga duración.

- Consumir carbohidratos de absorción rápida cuando sea necesario para evitar cuadros de hipoglucemia.

- Identificar el impacto de la hiperglucemia e hipoglucemia durante la actividad física.

- Recomendar llevar una identificación que indique la condición de paciente con DM.

- Evitar el ejercicio en el pico máximo de acción de la insulina.

- Prevenir la deshidratación con un consumo adecuado de líquidos antes, durante y después del ejercicio.

- Las dosis de insulina deben calcularse de acuerdo con la hora del día en que el ejercicio se realizará, y la reducción debe prioritariamente considerar la insulina de acción rápida.

- En la eventualidad de no ser posible una reducción de insulina, debe aumentarse la ingesta de hidratos de carbono antes, durante y después del entrenamiento de acuerdo con la glucemia.

- El paciente debe tener siempre a mano aparatos o útiles medidores de glucosa, así como hidratos de carbono de rápida absorción para evitar una hipoglucemia.

- No inyectar insulina en las extremidades cuando se realice ejercicio pues podrá haber una absorción más rápida. La región más indicada en este caso es el tejido subcutáneo de la pared abdominal.

- Si la actividad física se realizará por la tarde, el paciente debe reducir la insulina vespertina y aumentar la ingesta. Conviene pedir a los familiares que lo observen eventualmente durante el sueño para detectar eventuales hipoglucemias post ejercicio.

Cuadro 1: Lineamientos generales para la realización de actividad física. 
Revista de la Sociedad Argentina de Diabetes Año 55 Vol. 55 № 1 Suplemento Consenso Sociedad Argentina de CardiologíaSociedad Argentina de Diabetes Enero-abril de 2021: 03-70 ISSN 0325-5247 (impresa) ISSN 2346-9420 (en línea)

\section{BIBLIOGRAFÍA}

1. American Diabetes Association. Lifestyle management: Standards of Medical Care in Diabetes-2019. Diabetes Care 2019; 42(Suppl 1):S46-S60

2. Pastors JG, Warshaw H, Daly A, Franz M, Kulkarni K. The evidence for the effectiveness of medical nutrition therapy in diabetes management. Diabetes Care 2002; 25(3):608-613.

3. Evert A, Franz M. NutritionTherapy for diabetes and lipid disorders. American Diabetes Association. Virginia 2012; 265-294.

4. Knowler WC, Fowler SE, et al; Diabetes Prevention Program Research Group. 10-year follow-up of diabetes incidence and weight loss in the Diabetes Prevention Program Outcomes Study. Lancet 2009; 374(9702):1677-1686.

5. Willett WC. Dietary fats and coronary heart disease. J Intern Med 2012; 272(1):13-24.

6. Michas G, Micha R, Zampelas A. Dietary fats and cardiovascular disease: putting together the pieces of a complicated puzzle. Atherosclerosis 2014; 234(2):320-328.
7. Cosentino F, Grant PJ, Aboyans V, Bailey CJ, Ceriello A, Delgado V et al; ESC Scientific Document Group. 2019 ESC Guidelines on diabetes, pre-diabetes, and cardiovascular diseases developed in collaboration with the EASD. Eur Heart J 2020; 41(2):255-323.

8. Brand-Miller J, Hayne S, Petocz P, Colagiuri S. Low-glycemic index diets in the management of diabetes: a meta-analysis of randomized controlled trials. Diabetes Care 2003; 26(8):2261-2267.

9. Thomas DE, Elliott EJ. The use of low-glycaemic index diets in diabetes control. Br J Nutr 2010; 104(6):797-802.

10. Knowler WC, Barrett-Connor E, Fowler SE, Hamman RF, Lachin JM, Walker EA, et al; Diabetes Prevention Program Research Group. Reduction in the incidence of type 2 diabetes with lifestyle intervention or metformin. N Engl J Med 2002; 346(6):393-403.

11. Aguilar-Salinas CA, Aschner P, González R, Mora-Brito E, Rodríguez M, Domínguez $E$, et al. Guías ALAD sobre el diagnóstico, control y tratamiento de la diabetes mellitus tipo 2 con medicina basada en evidencia. Edición 2019. Revista de la Asociación Latinoamericana de Diabetes 2019;1-125.

\section{Tratamiento farmacológico de la hiperglucemia}

\section{a. Objetivos glucémicos: intensivo vs conservador}

Hace varios años el estudio United Kingdom Prospective Diabetes Study (UKPDS) demostró que mantener un adecuado control glucémico con valores de $\mathrm{HbA} 1 \mathrm{c}$ menores del $7 \%$ permite reducir las complicaciones microvasculares pero no garantiza la reducción de eventos macrovasculares'. Los ensayos clínicos posteriores como el Action in Diabetes and Vascular Disease (ADVANCE), Action to Control Cardiovascular Risk in Diabetes (ACCORD) y Veterans Affairs Diabetes Trial (VADT) confirmaron que ser más intensivos en el control glucémico no implica necesariamente una reducción de muerte por causas cardiovasculares debido, probablemente, a un evento adverso asociado a ciertos fármacos: la hipoglucemia ${ }^{2-4}$. Muchas veces alcanzar objetivos de control glucémico más estrictos implica asociar fármacos con mayor riesgo de desarrollar hipoglucemias como las sulfonilureas, glinidas e insulinoterapia. En los últimos años surgieron numerosas evidencias sobre el impacto favorable CV y renal de nuevos grupos farmacológicos utilizados para el control glucémico del paciente con DM tipo 2: los iSGLT-2 y los arGLP-1. El advenimiento de estos fármacos plantea la importancia de considerar no sólo objetivos de $\mathrm{HbA} 1 \mathrm{c}$, sino la adecuada selección de los fármacos para alcanzar dichas metas.

\section{Metas de control glucémico}

Los objetivos de control glucémico se basan en la individualización de la $\mathrm{HbA} 1 \mathrm{c}$ :

- HbA1c menor del 7\%: el logro de este objetivo demostró asociarse a una reducción de complicaciones microvasculares. Si se implementa poco tiempo después del diagnóstico de DM, se asocia también a una reducción a largo plazo de las complicaciones cardiovasculares independientemente del fármaco utilizado.

- HbA1c menor del 6,5\%: estos objetivos pueden asociarse a un menor riesgo de complicaciones microvasculares, sobre todo de nefropatía diabética, cuando se comparan con objetivos de $\mathrm{HbA} 1 \mathrm{c}<7 \%$. Sin embargo, el factor limitante es la necesidad de utilizar fármacos con mayor riesgo de hipoglucemia para alcanzar estos objetivos. Se sugiere evaluar objetivos más estrictos en aquellos pacientes que no presenten riesgo de hipoglucemia: adultos jóvenes, DM de corta duración, expectativa de vida larga y ausencia de comorbilidades significativas.

- HbA1c menor del 8\%: se sugiere este objetivo en aquellos pacientes que han experimentado hipoglucemias frecuentes o en aquellos vulnerables o frágiles por edad avanzada y condiciones comórbidas que puedan limitar la expectativa de vida, y en pacientes con DM de larga data y múltiples complicaciones, en quienes el logro de metas es difícil a pesar de una óptima educación o a quienes no les sea posible realizar automonitorización.

Asimismo, deben tenerse en cuenta otros parámetros secundarios, como alcanzar una glucemia en ayu- 
Revista de la Sociedad Argentina de Diabetes Año 55 Vol. 55 № 1 Suplemento Consenso Sociedad Argentina de CardiologíaSociedad Argentina de Diabetes Enero-abril de 2021: 03-70 ISSN 0325-5247 (impresa) ISSN 2346-9420 (en línea)

nas entre $80 \mathrm{mg} / \mathrm{dL}$ y $130 \mathrm{mg} / \mathrm{dL}$, y una glucemia posprandial (a los 60 a 120 minutos) menor de $180 \mathrm{mg} / \mathrm{dL}$.

\section{Recomendaciones}

- Mantener un adecuado control glucémico ( $\mathrm{HbA} 1 \mathrm{C}<7 \%)$ con el objetivo de reducir el riesgo de complicaciones microvasculares (Grado de recomendación I. Nivel de evidencia A).

- Objetivos glucémicos más o menos estrictos deberán individualizarse de acuerdo con las características y comorbilidades de los pacientes (Grado de recomendación I. Nivel de evidencia C).

- Se recomienda evitar el desarrollo de hipoglucemia (Grado de recomendación I. Nivel de evidencia C).

\section{b. Mecanismo de acción y seguridad cardiovascular de los fármacos}

La relación entre el tratamiento farmacológico antidiabético y la enfermedad CV encontró en varias ocasiones puntos de controversia. Se sabe que dos tercios de los pacientes con DM presentan enfermedad cardiovascular, siendo ésta la principal causa de muerte ${ }^{5}$. Sin embargo, durante muchos años, con el tratamiento dirigido al control glucémico y la prevención de sus complicaciones, no se observó reducción en estos eventos e incluso se dudó en muchos casos de su seguridad cardiovascular. Las sulfonilureas (SU) se asociaron a toxicidad cardiovascular, fundamentalmente ligadas a su mecanismo de acción en los canales voltaje dependientes de potasio, con potencial incremento de muerte celular durante la isquemia, de aumento de área de infarto y de arritmias ventriculares ${ }^{6-8}$. Por otra parte, la fenformina se asoció a acidosis láctica, principalmente en la insuficiencia renal y en la insuficiencia cardíaca (IC), lo que motivó su retiro del mercado y el retraso de la llegada de la metformina. Con estos antecedentes, en el año 2007 se publicaron dos metaanálisis sobre tiazolidindionas describiendo su capacidad de generar fallo de bomba y probable aumento de infarto de miocardio, y una tendencia al aumento de mortalidad cardiovascular, trabajos que motivaron a la Food and Drug Administration (FDA) de los Estados Unidos, en 2008, a exigir seguridad cardiovascular a todos los fármacos antidiabéticos futuros. Ello generó una serie de estudios que marcó grandes avances con respecto al entendimiento de la seguridad cardiovascular de los nuevos fármacos antidiabéticos. En los próximos apartados se mencionan los principales aspectos de eficacia y seguridad cardiovascular de los grupos farmacoló- gicos utilizados, pero excede el propósito de este texto la descripción pormenorizada de la farmacología clínica y la farmacovigilancia de cada uno.

\section{Sulfonilureas}

El mecanismo de acción de estos fármacos se centra en la estimulación de la secreción de insulina mediante la unión de receptores específicos de la célula $\beta$, que deriva en el cierre de canales de $K_{+}$ dependientes de ATP con la consiguiente apertura de los canales de $\mathrm{Ca}+$ dependientes de voltaje y la exocitosis de los gránulos de insulina. Los canales de ATP están compuestos de dos subunidades, la Kir 6.2 y el SUR, que es el sitio de unión a las SU. Los SUR1 se encuentran predominantemente en las células $\beta$, los SUR2A en el músculo cardíaco y los SUR2B en el músculo liso. La apertura de los canales de $\mathrm{K}+$ dependientes de ATP en el miocardio se considera protectora durante períodos de isquemia. Por lo tanto, las SU no selectivas, con afinidad para estos canales a nivel cardíaco, podrían inhibir este mecanismo protector ${ }^{9}$.

Dentro de esta familia de fármacos debemos diferenciar las más antiguas de las modernas SU, sobre todo la gliclazida, que se caracteriza por generar un menor aumento de peso, posee una mayor selectividad por los receptores de la célula $\beta$ y una menor tasa de hipoglucemias cuando se la compara con el resto de la familia por presentar una unión más reversible al receptor ${ }^{10-12}$. Otro efecto beneficioso de la gliclazida es que posee propiedades de scavenger (barredores) de radicales libres, lo que podría proteger a la célula $\beta$ de la apoptosis mediada por radicales libres ${ }^{12}$. Algunos estudios evidenciaron que la gliclazida posee propiedades antiateroscleróticas mediadas por la activación de la proteína quinasa activadora de AMP (AMPK), aunque se desconoce el impacto clínico. Por otra parte, al no poseer metabolitos activos, se pueden utilizar con filtrados renales más bajos que el resto de la familia. La glibenclamida, por un lado, es menos selectiva e hipotéticamente puede actuar también a nivel miocárdico, sumado a que posee una unión al receptor que revierte lentamente con mayor riesgo de desarrollar hipoglucemia ${ }^{13}$. La glimepirida, si bien al igual que la glibenclamida es menos selectiva sobre los canales SU, parecería no incrementar eventos vasculares. La seguridad cardiovascular de la glimepirida fue evaluada en el estudio CARdiovascular Outcome Study of LINAgliptin versus glimepiride in patients with type 2 diabetes (CAROLINA). El ensayo clínico que reclu- 
tó 6.033 pacientes no observó aumento de eventos cardiovasculares con glimepirida en un seguimiento a 6,3 años en comparación con linagliptina. Un metaanálisis de 13 estudios con SU y 14 agentes antidiabéticos orales sin SU (16.260 pacientes) evidenció reducciones comparables en $\mathrm{HbA} 1 \mathrm{C}$ entre las SU, pero el riesgo de hipoglucemia fue inferior con gliclazida en comparación con glipizida (OR 0,22, IC: 0,05 a 0,96), glimepirida (OR 0,40, IC: 0,13 a 1,27 ) y glibenclamida (OR 0,21, IC: 0,03 a 1,48 ). Con respecto a la seguridad cardiovascular, la gliclazida parece no asociarse a un mayor riesgo de eventos cardiovasculares. Por ejemplo, los resultados de mortalidad cardiovascular evidencian que usando la glibenclamida como comparador, el riesgo relativo para gliclazida fue de 0,60 (95\% IC 0,45-0,84), para glimepirida de $0,79(0,57-1,11)$, para glipizida de 1,01 $(0,72-1,3)$ y $1,45(0,88-2,44)$ para clorpropamida ${ }^{14}$. Por lo tanto, debemos considerar este grupo de fármacos como una familia heterogénea, evitar el uso de glibenclamida en general y de requerir el uso de estos grupos farmacológicos, utilizar SU con un perfil más seguro y con menor riesgo de hipoglucemia como la gliclazida o glimepirida, sobre todo en pacientes con ECV establecida ${ }^{15}$.

\section{Metformina}

Ejerce su acción principal a nivel hepático, inhibiendo la neoglucogénesis y la glucogenólisis a través de su acción sobre el complejo 1 de la cadena respiratoria mitocondrial y la consecuente activación de la proteína quinasa activadora de AMP (AMPK, sus siglas en inglés), su mecanismo de acción más importante. Disminuye la glucemia, favorece el descenso de la hiperinsulinemia, mejora el perfil lipídico, el peso corporal y el aumento de la fibrinólisis. Recientemente se ha mencionado que la metformina podría aumentar la síntesis del GLP-1, generando así un efecto secretagogo indirecto a nivel pancreático. No produce hipoglucemia. Se elimina por vía renal $(90 \%)$ y correlaciona en forma estrecha con el filtrado glomerular ${ }^{16}$. El estudio Diabetes Prevention Program (DPP) demostró inicialmente que la metformina en pacientes con tolerancia alterada a los hidratos de carbono y glucemia alterada en ayunas disminuye la incidencia de DM en un 31\% comparado con placebo ${ }^{17}$. Recientemente, 14 años después de la aleatorización de este estudio, se midió el contenido de calcio coronario y los resultados evidenciaron que la metformina disminuyó el contenido de calcio coronario de forma significativa en hombres, com- parado con la rama asignada a placebo o a cambios en el estilo de vida ${ }^{18}$. Con respecto a la seguridad CV de la metformina, son numerosos los estudios observacionales que asocian su uso con un impacto favorable en end points cardiovasculares. Sin embargo, son pocos los ensayos clínicos que confirmaron estos hallazgos. El más representativo es el estudio UKPDS que mostró en la rama de tratamiento asignada a metformina, durante la investigación e incluso en una extensión luego de 10 años de finalizada, beneficios cardiovasculares asociados con su uso; allí se observó una reducción significativa del riesgo para cualquier end point relacionado con DM del $21 \%(p=0,01)$, para infarto agudo de miocardio (IAM) del 33\% $(p=0,005)$ y para muerte de cualquier causa del 27\% ( $p=0,002)$. Este beneficio a largo plazo de la metformina se evidenció en pacientes con sobrepeso ${ }^{19}$. Por su elevada eficacia en descender la HbA1c, por su seguridad en términos de efectos adversos, por su bajo costo y su probable impacto cardiovascular beneficioso, se considera a la metformina como el fármaco de primera elección al momento del diagnóstico de la DM2. También puede indicarse a pacientes con glucemia alterada en ayunas o tolerancia alterada a los hidratos de carbono, ya que en este grupo demostró retrasar o prevenir el diagnóstico de DM (en especial en pacientes menores de 60 años, IMC mayor de 35, o en mujeres con antecedentes de DM gestacional). Otro grupo para considerar son las mujeres con síndrome del ovario poliquístico, ya que logra mejorar la resistencia a la insulina propia de esta entidad. No se recomienda su indicación para el tratamiento de la insulinorresistencia sin disglucemia, ni para el tratamiento del sobrepeso u obesidad ${ }^{20}$.

\section{Tiazolindinedionas}

La rosiglitazona y pioglitazona interactúan con receptores nucleares PPAR gamma (peroxisome proliferator actived receptor), estimulando la transcripción de proteínas que regulan el metabolismo de los hidratos de carbono y de los lípidos, por ejemplo de los GLUT-4 (en músculo y tejido adiposo), de los ácidos grasos, de moléculas proinflamatorias como el TNF- $\alpha$ y la IL-6, etc. ${ }^{21,22}$. Sin embargo, los efectos pleitrópicos no pueden considerarse un efecto de clase, ya que la rosiglitazona fue asociada, en algunos metaanálisis, con un incremento del riesgo $\mathrm{CV}^{23}$, pese a que varios estudios posteriores no verificaron esta hipótesis ${ }^{24}$. De todas maneras, la European Medicines Agency (EMA) determinó suspender su 
comercialización ${ }^{25}$, la FDA ha notificado una alerta sobre su indicación y la Administración Nacional de Medicamentos, Alimentos y Tecnología Médica (ANMAT) sugiere su venta bajo receta archivada y la instrumentación de un plan de farmacovigilancia para este fármaco ${ }^{26}$, aunque la evidencia posteriormente publicada no confirmó esa sospecha. Un metaanálisis publicado en 2017 analizó nueve estudios aleatorizados y controlados para evaluar la relación entre pioglitazona y enfermedad CV. Los resultados evidenciaron que el agregado de pioglitazona a la terapia estándar reduce el punto final combinado de eventos cardiovasculares mayores (MACE, por sus siglas en inglés) en un 17\% en pacientes con DM2. Sin embargo, diversos eventos adversos relacionados con el uso de estos fármacos -como mayor riesgo de internación por insuficiencia cardíaca y edema asociado a retención hidrosalina, fracturas y aumento de peso- limitan en muchas ocasiones su uso ${ }^{18,27}$.

\section{Inhibidores DPP-4}

Son fármacos de administración oral que, al inhibir a la enzima dipeptidil peptidasa-4 (DPP-4), evitan la degradación de GLP-1. Su eficacia en términos de reducción de $\mathrm{HbA} 1 \mathrm{c}$ oscila entre 0,4 y $0,8 \%$. Su efecto sobre el peso suele ser neutro $y$, por lo general, son fármacos bien tolerados. En lo que respecta a los resultados cardiovasculares de estos estudios podemos mencionar el estudio Saxagliptin Assessment of Vascular Outcomes Recorded (SAVOR) ${ }^{28}$ con saxagliptina que enroló pacientes tanto en prevención primaria como secundaria que resultó no inferior a placebo para el objetivo combinado de MACE; sin embargo, se observó un aumento de las hospitalizaciones por insuficiencia cardíaca. Por su parte, el estudio Examination of Cardiovascular Outcomes with Alogliptin (EXAMINE) ${ }^{29}$ con alogliptina incluyó pacientes más agudos puesto que tenían antecedentes de internación por síndrome coronario agudo dentro de los 15 a 90 días previos. En esta población también se evidenció la no inferioridad con respecto al tratamiento estándar para MACE y un aumento no significativo de las hospitalizaciones por IC. El estudio Trial Evaluating Cardiovascular Outcomes with Sitagliptin (TECOS) ${ }^{30}$, que puso a prueba la sitagliptina en 14.671 pacientes en prevención secundaria, también resultó no inferior con relación a su grupo control en su objetivo combinado de muerte $C V$, IAM, ACV e internaciones por angina inestable, pero resultó neutro en internaciones por IC. En el estudio Cardiovascular and Renal Microvascular Outcome Study With Linagliptin in Patients with Type 2 Diabetes Mellitus (CARMELINA) ${ }^{31}$, que evaluó la eficacia y seguridad de la linagliptina en una población con alto riesgo cardiovascular y con alta prevalencia de insuficiencia renal, se evidenció no inferioridad en términos de eficacia ni aumento de eventos cardiovasculares. Podríamos resumir que son fármacos seguros desde el punto de vista cardiovascular, excepto el mayor riesgo de internación por insuficiencia cardíaca observado exclusivamente con saxagliptina (Figura 1) y la presencia de efectos adversos extremadamente muy infrecuentes, como enfermedad inflamatoria intestinal, pénfigo y artralgias.

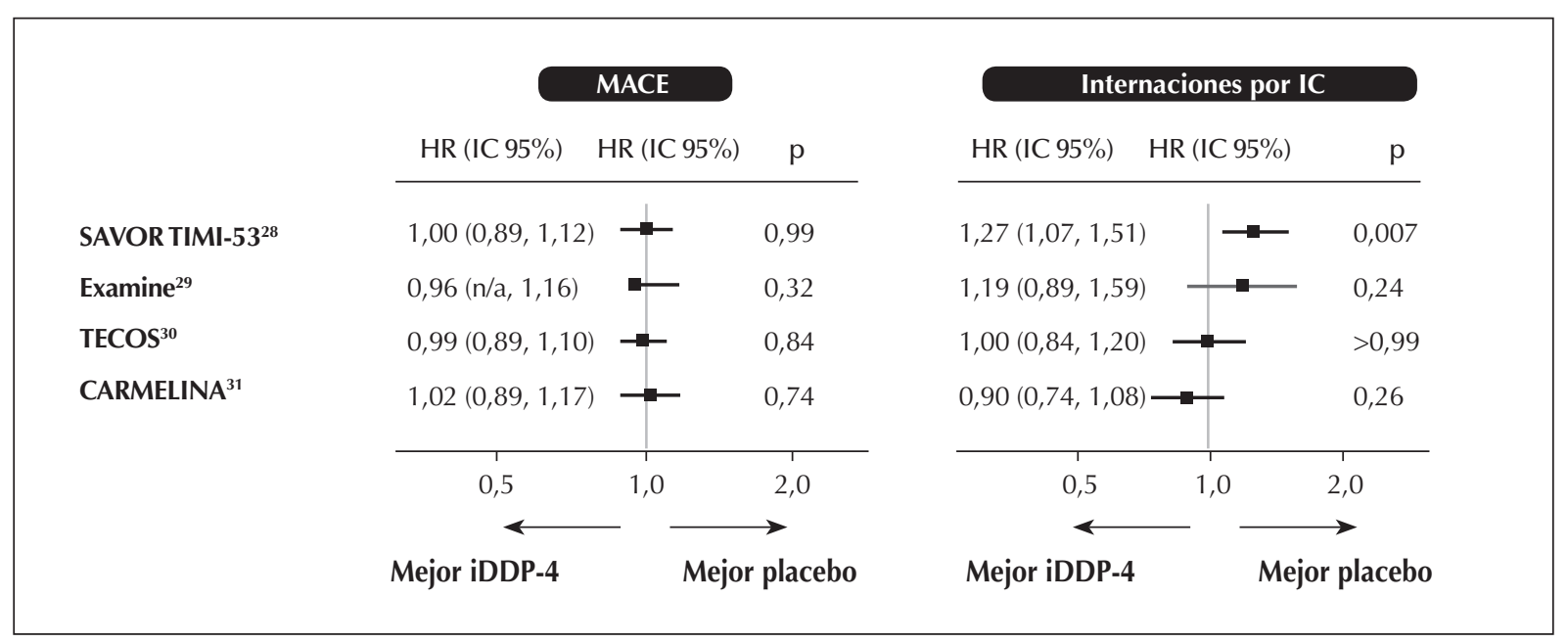

MACE: eventos cardiovasculares mayores; IC: insuficiencia cardíaca.

Figura 1: Ensayos clínicos evaluando impacto cardiovascular con inhibidores DPP-4. 


\section{Agonistas del receptor GLP-1}

Los agonistas de los receptores de GLP-1 (g/ucagon like peptide-1), a través de un mecanismo dependiente de la glucosa, aumentan la secreción de insulina y reducen la de glucagón. Por el momento se dispone de formulaciones de administración subcutánea y existen importantes diferencias en la estructura farmacológica y tiempo de duración de su efecto entre los distintos fármacos que conforman este grupo, las cuales podrían explicar los diferentes efectos farmacológicos y clínicos entre ellos. Los fármacos de este grupo, además de generar una reducción en la HbA1c, producen entre otros efectos positivos, pérdida ponderal (2-4 kg), en parte por acción directa a nivel del sistema nervioso central disminuyendo el apetito, y reducción de la presión arterial por efectos natriuréticos y modificaciones a nivel lipídico (reducción TG y aumento C-HDL). Estos fármacos fueron extensamente evaluados en estudios de seguridad cardiovascular. El estudio Evaluation of LIXisenatide in Acute coronary syndrome, (ELIXA) ${ }^{32}$ aleatorizó pacientes que habían experimentado dentro de los seis meses previos un evento coronario agudo y, si bien no pudo demostrar superioridad frente a placebo, sí resultó no inferior en el combinado de muerte, ACV, IAM o internaciones por angina inestable. En contraste con estos resultados, un año después se presentó el estudio Liraglutide and Cardiovascular Outcomes in Type 2 Diabetes (LEADER) ${ }^{33}$ con liraglutida, que incluyó pacientes en prevención primaria $(19 \%)$ y secundaria $(81 \%)$, y alcanzó superioridad con respecto a placebo en el combinado de muerte CV, IAM no fatal y ACV no fatal (reducción $13 \% p=0,001)$. Particularmente, liraglutida redujo significativamente la muerte CV $(22 \% p=0,007)$ y la muerte por cualquier causa $(15 \% p=0,02)$, sin efectos sobre las internaciones por IC. En este sentido, el semaglutida en el estudio Sustainability in Treatment of Type 2 Diabetes (SUSTAIN-6) $)^{34}$, con una población en un $83 \%$ en prevención secundaria, también demostró reducir con significancia estadística el compuesto de MACE en un $26 \%(p=0,02)$, el compuesto de MACE ampliado con revascularización periférica o coronaria, internaciones por angina inestable o IC y el compuesto de muerte de cualquier causa, ACV e IAM. En el análisis de cada componente en forma individual, la molécula redujo la necesidad de revascularización en un $35 \%(p=0,003)$ y la incidencia de ACV no fatal 39\% $(p=0,04)$ sin reducción en internaciones por IC. Por su parte, albiglutida en el estudio Albiglutide and Cardiovascular Outcomes in Patients with Type 2 Diabetes and Cardiovascular Disease (HARMONY) ${ }^{35}$ fue capaz de disminuir significativamente el MACE, el MACE ampliado con revascularización y el IAM, sin influencia en ACV y mortalidad total ni CV. Por último, el estudio Researching Cardiovascular Events with a Weekly Incretin in Diabetes (REWIND) ${ }^{36}$, a diferencia de los anteriores, evaluó una población principalmente en prevención primaria $(69,5 \%)$. En éste, dulaglutida fue superior a placebo con una reducción del $12 \%$ en MACE $(p=0,026)$, observándose además una reducción en $A C V$ no fatal $24 \%(p=0,017)$ sin impacto en mortalidad total o CV. De esta manera, puede apreciarse que los arGLP-1 no parecen integrar una clase homogénea a la hora de evaluar los resultados. Por otra parte, entre los arGLP-1, con efecto favorable desde el punto de vista cardiovascular, los resultados observados son independientes del manejo de los factores de riesgo; su impacto clínico es mayor en poblaciones con DM con eventos previos (Figura 2).

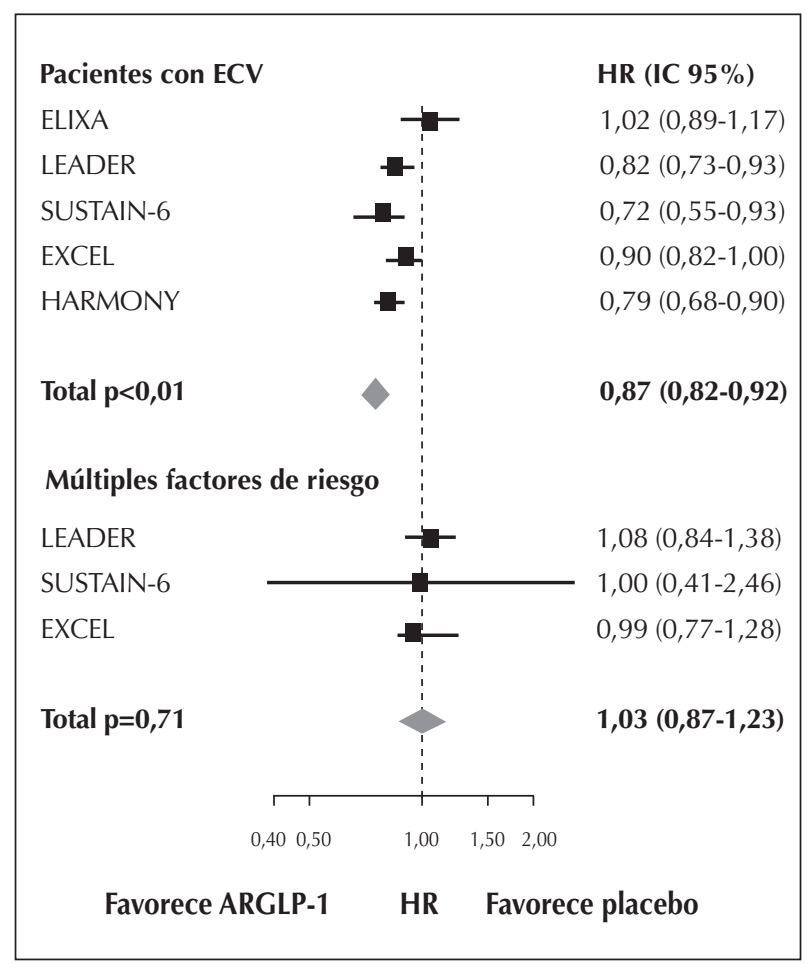

ECV: enfermedad cardiovascular.

Figura 2: Ensayos clínicos con agonistas de receptores GLP-1 evaluando impacto cardiovascular 


\section{Inhibidores del cotransportador SGLT-2}

Como su nombre lo indica, inhiben el cotransportador sodio-glucosa tipo 2 ubicado a nivel del túbulo proximal generando glucosuria y natriuresis. Reducen HbA1c entre 0,4-0,8\% promedio, generan pérdida ponderal, reducción de la presión arterial sistólica y diastólica, y en los estudios clínicos demostraron resultados positivos a nivel cardiovascular y renal con un efecto de clase más ostensible y extendido que los agonistas de receptores GLP-137. El primer estudio de seguridad CV sobre este grupo en publicarse en el año 2015 fue el Empagliflozin Cardiovascular Outcome Event Trial in Type 2 Diabetes Mellitus Patients (EMPA-REG) ${ }^{38}$ que, en una población con patología cardiovascular establecida, demostró que el uso de empagliflozina se asoció a una reducción significativa de MACE $14 \%(p=0,04)$, de muerte CV 38\% $(p<0,001)$, de muerte de cualquier causa $32 \%(p<0,001)$ y de las internaciones por IC $35 \%$ $(p=0,002)$. Posteriormente, el estudio Canagliflozin Cardiovascular Assessment Study (CANVAS) ${ }^{39}$ con canagliflozina demostró, en una población menos enferma con un $40 \%$ aproximadamente en prevención primaria, reducción significativa de MACE $14 \%(p=0,02)$ y de las hospitalizaciones por IC 33\% ( $p=0,2)$, al tiempo que evidenció beneficios en materia de protección renal (menor progresión o regresión de microalbuminuria, menor caída del filtrado glomerular, necesidad de terapia de sustitución renal y muerte de causa renal). Al igual que lo observado con los agonistas de re- ceptores GLP-1, extendiendo la evidencia a prevención primaria, el estudio Dapagliflozin Effect on Cardiovascular Events (DECLARE) ${ }^{40}$ incluyó 17.160 pacientes, de los cuales el $60 \%$ no presentaba patología cardiovascular establecida. En ese estudio no hubo reducción de MACE. Dapagliflozina fue superior en términos del combinado de mortalidad cardiovascular/hospitalizaciones por IC con una reducción del $17 \%(p=0,005)$ y se ratificó la reducción de internaciones por insuficiencia cardíaca en un $27 \%$, así como los efectos nefroprotectores de la molécula. Estos beneficios se observaron tanto en la población con factores de riesgo como en pacientes con enfermedad CV establecida, y en los tres estudios mencionados fue independiente del antecedente de insuficiencia cardíaca previo a la inclusión en el estudio (Figura 3). Entre los efectos adversos informados, este grupo farmacológico se asoció con un aumento significativo de infecciones genitales (no así urinarias) y se ha referido riesgo de hipotensión ortostática en algunos grupos específicos (adultos mayores, uso concomitante de diuréticos y disautonomía). En el estudio CANVAS se informó un aumento en la incidencia de amputaciones a nivel de miembros inferiores, que no fue evidenciado en los otros estudios mencionados ni en el estudio Canagliflozin and Renal Events in Diabetes with Established Nephropathy Clinical Evaluation (CREDENCE) ${ }^{41}$, que evaluó la utilización de canagliflozina en una población con deterioro de la función renal.

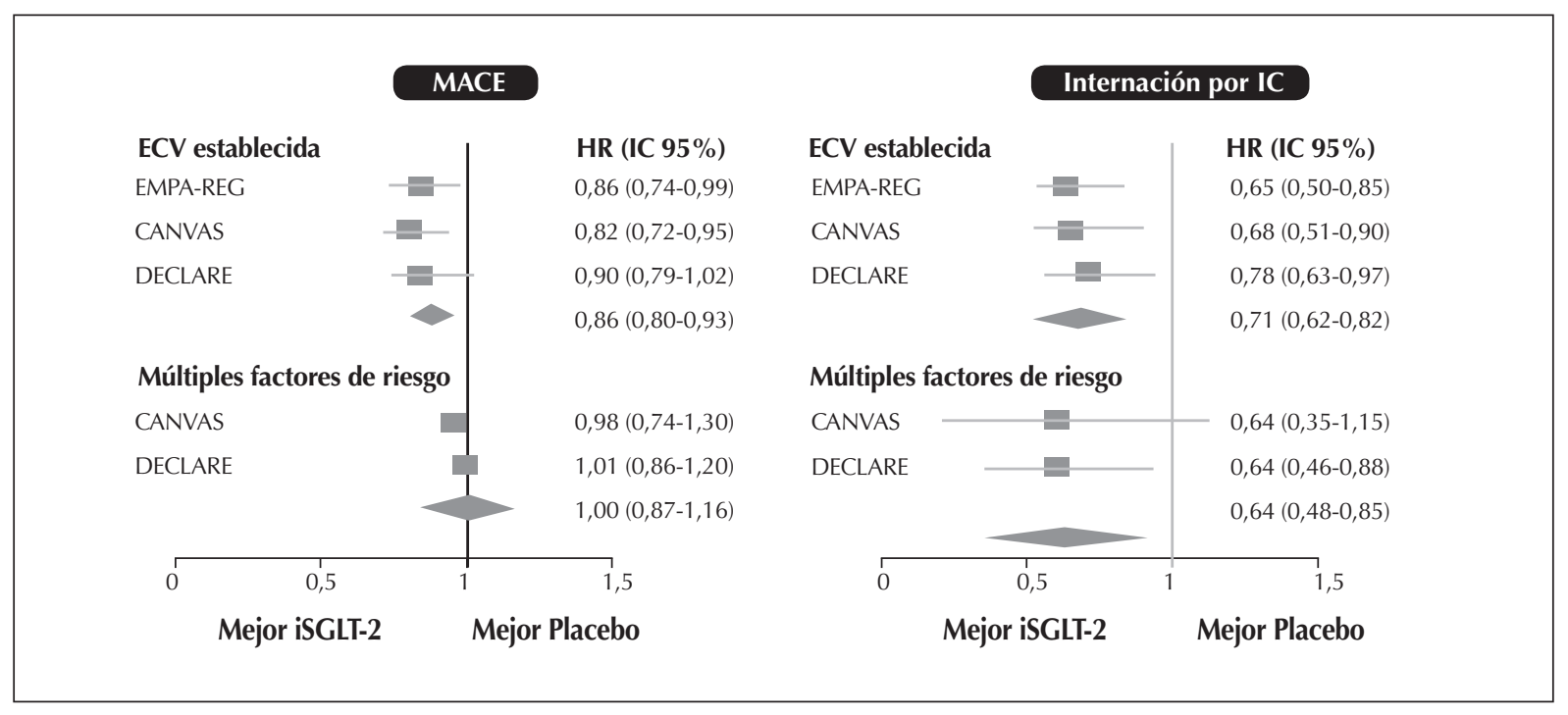

MACE: eventos cardiovasculares mayores; IC: insuficiencia cardíaca; ECV: enfermedad cardiovascular.

Figura 3: Ensayos clínicos con inhibidores de SGLT-2 evaluando impacto cardiovascular. 
Revista de la Sociedad Argentina de Diabetes Año 55 Vol. 55 № 1 Suplemento Consenso Sociedad Argentina de CardiologíaSociedad Argentina de Diabetes Enero-abril de 2021: 03-70 ISSN 0325-5247 (impresa) ISSN 2346-9420 (en línea)

\section{Insulinoterapia}

El empleo correcto de la insulina depende de una serie de cuidados que deben tener en cuenta el médico tratante y el paciente, sobre todo en la prevención y el tratamiento de las hipoglucemias. Es de gran importancia recordar que la insulina debe guardarse refrigerada. Luego de abierto el frasco o de comenzado el cartucho, no debe utilizarse más de 28 días ya que existen diferencias sustanciales de su actividad si se la emplea por más tiempo. La insulina de uso diario puede estar a temperatura ambiente (hace menos dolorosa la aplicación), pero protegida de la luz ${ }^{42}$. La insulina es una proteína de bajo peso molecular. En la gran mayoría de los países se la envasa en concentraciones de $100 \mathrm{UI} / \mathrm{mL}$, aunque para situaciones especiales de insulinorresistencia puede obtenerse en concentraciones de 200, 300 ó $500 \mathrm{UI} / \mathrm{mL}$. Las insulinas pueden clasificarse por su origen y su tiempo de acción. Las insulinas humanas son producidas por tecnología de ADN recombinante ${ }^{43}$.

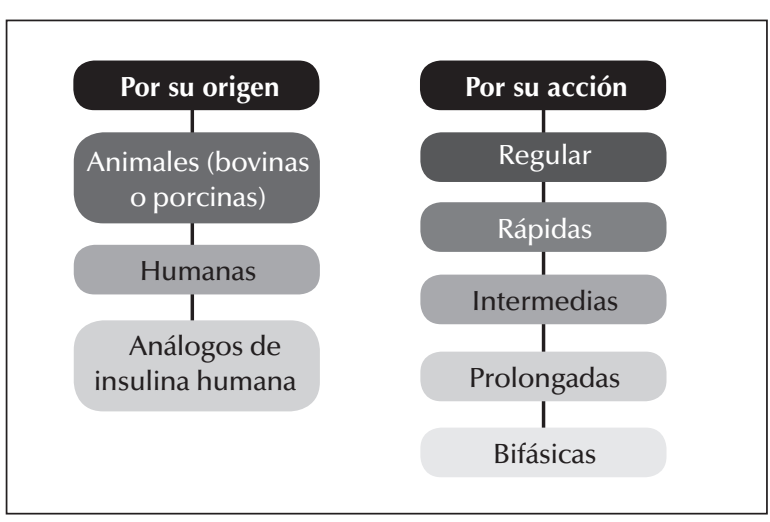

Figura 4: Clasificación de insulinas.

\begin{tabular}{|c|c|c|}
\hline Duración de la acción & Tiempo de inicio de acción & Tiempo hasta el pico de acción \\
\hline \multicolumn{3}{|l|}{ Insulinas de acción rápida } \\
\hline $\begin{array}{l}\text { Insulina humana regular } \\
\text { 5-8 h }\end{array}$ & $30-60 \min$ & $30-60 \min$ \\
\hline $\begin{array}{l}\text { Aspártica } 1 \\
3-5 \text { h }\end{array}$ & $2-18 \min$ & $0-90 \min$ \\
\hline $\begin{array}{l}\text { Glulisina } \\
3-5 \mathrm{~h}\end{array}$ & $12-30 \mathrm{~min}$ & $30-90 \mathrm{~min}$ \\
\hline $\begin{array}{l}\text { Lispro } \\
3-5 \mathrm{~h} \\
\end{array}$ & $15-30 \mathrm{~min}$ & $30-90 \mathrm{~min}$ \\
\hline \multicolumn{3}{|l|}{ Insulinas de acción intermedia } \\
\hline $\begin{array}{l}\mathrm{NPH} \\
12-16 \mathrm{~h} \\
\end{array}$ & $1-2 \mathrm{~h}$ & $4-12 \mathrm{~h}$ \\
\hline $\begin{array}{l}\text { Lispro protamina } \\
12-16 \mathrm{~h}\end{array}$ & $30-60 \mathrm{~min}$ & $4-12 \mathrm{~h}$ \\
\hline \multicolumn{3}{|l|}{ Insulinas de acción prolongada y ultraprolongada } \\
\hline $\begin{array}{l}\text { Detemir } \\
\text { Hasta } 24 \mathrm{~h} \\
\end{array}$ & $1-2 \mathrm{~h}$ & $6-8 \mathrm{~h}$ \\
\hline $\begin{array}{l}\text { Glargina U100 } \\
20-26 \mathrm{~h}\end{array}$ & $1-2 \mathrm{~h}$ & Ninguna \\
\hline $\begin{array}{l}\text { Glargina U300* } \\
\text { Hasta } 36 \mathrm{~h}\end{array}$ & $1-2 \mathrm{~h}$ & Ninguna \\
\hline $\begin{array}{l}\text { Degludec* } \\
>42 \mathrm{~h}\end{array}$ & $30-90 \min$ & Ninguna \\
\hline \multicolumn{3}{|l|}{ Bifásicas o premezclas } \\
\hline $\begin{array}{l}70 \% \mathrm{NPH}, 30 \% \text { regular } \\
10-16 \mathrm{~h}\end{array}$ & $30-60 \mathrm{~min}$ & $2-4 \mathrm{~h}$ \\
\hline $\begin{array}{l}70 \% \text { aspártica protamina } 30 \% \\
\text { aspártica } \\
10-16 \mathrm{~h}\end{array}$ & $15-30 \mathrm{~min}$ & $1-12 \mathrm{~h}$ \\
\hline $\begin{array}{l}75 \% \text { lispro protamina } 25 \% \text { lispro } \\
10-16 \mathrm{~h}\end{array}$ & $10-15 \min$ & $1-12 \mathrm{~h}$ \\
\hline $\begin{array}{l}50 \% \text { lispro protamina } 50 \% \text { lispro } \\
10-16 \mathrm{~h}\end{array}$ & $10-15 \min$ & $1-4 h$ \\
\hline
\end{tabular}

* Ultraprolongada.

Tabla 1: Características farmacológicas de las insulinas. 
- Insulina y seguridad cardiovascular: los resultados del estudio Outcome Reduction with an Initial Glargine Intervention (ORIGIN), realizado con insulina glargina U100 utilizada para conseguir niveles normales de glucosa plasmática en ayunas durante más de seis años, tuvo un efecto neutro sobre los eventos cardiovasculares y cánceres. Se observó aumento de hipoglucemia y leve incremento de peso $^{44}$. El estudio Trial Comparing Cardiovascular Safety of Insulin Degludec versus Insulin Glargine in Patients with Type 2 Diabetes at High Risk of Cardiovascular Events (DEVOTE) demostró menor incidencia y tasa de hipoglucemia severa y severa nocturna con insulina degludec vs insulina glargina U10045. Estos hallazgos son consistentes con los resultados en el estudio Trial Comparing the Safety and Efficacy of Insulin Degludec and Insulin Glargine, both with Insulin Aspart as Mealtime Insulin in Subjects With Type 1 Diabetes (SWITCH).

- Hipoglucemias: el tratamiento con insulina incrementa el riesgo de hipoglucemias, razón por la cual, en paciente con ECV o elevado riesgo cardiovascular, se debe evitar dado que puede desencadenar varias respuestas que involucran múltiples vías de estrés y activación del sistema nervioso simpático que conduce a efectos hemodinámicos, proinflamatorios y efectos proaterotrombóticos, además de aumentar el potencial de arritmias cardíacas ${ }^{46}$. También estimula un aumento de la aldosterona plasmática a través de la activación del sistema renina-angiotensina que, por la activación del receptor mineralocorticoide, puede exacerbar la disfunción endotelial.

\section{c. Algoritmos de manejo de la hiperglucemia de acuerdo con el riesgo cardiovascular}

NOTA: queda a consideración del médico tratante verificar las regulaciones, incluido el acceso a los medicamentos aplicables para cada tipo de cobertura de salud.

\section{Manejo de la hiperglucemia del paciente con DM2 y enfermedad cardiovascular aterosclerótica}

Se entiende por enfermedad cardiovascular aterosclerótica la presencia de cardiopatía isquémica en todas sus formas de presentación (síndromes coronarios agudos, angina crónica estable y antecedente de revascularización coronaria), el accidente cerebrovascular isquémico, la presen- cia de arteriopatía periférica (carotídea, aórtica, de miembros inferiores), ya sea revascularizada o no.

Se definió como primer escalón de tratamiento independientemente de los niveles de $\mathrm{HbA} 1 \mathrm{c}$ la implementación de modificaciones del estilo de vida, sobre el cual se efectuará el tratamiento farmacológico. Este depende de los niveles de $\mathrm{HbA} 1 \mathrm{c}$ de inicio quedando conformados tres grupos de pacientes: 1) HbA1c inicio $<7 \%$; 2) HbA1c inicio $>7 \%$ y $<9 \%$; 3) $\mathrm{HbA} 1 \mathrm{c}$ inicio $>9 \%$.

\section{HbA1c inicio $<7 \%$}

- En este grupo se sugiere iniciar monoterapia con metformina (Grado de recomendación I. Nivel de evidencia C) y considerar adicionar tempranamente fármacos con beneficio cardiovascular demostrado: iSGLT-2 o arGLP-1 (Grado de recomendación I. Nivel de evidencia C).

- En caso de no poder recibir metformina, se sugiere utilizar monoterapia con fármacos con beneficio CV demostrado (iSGLT-2 o arGLP-1) (Grado de recomendación Ila. Nivel de evidencia B).

- De no poder utilizar fármacos con beneficio CV demostrado, se sugiere la utilización de iDPP-4, tiazolidinedionas (considerando el riesgo de insuficiencia cardíaca), sulfonilureas (no utilizar glibenclamida) (Grado de recomendación I. Nivel de evidencia C).

- En pacientes seleccionados se podrá incorporar tempranamente la asociación con iSGLT-2 o arGLP-1 (Grado de recomendación I. Nivel de evidencia C).

De no alcanzarse objetivos de A1C en tres meses, continuar con el esquema propuesto en el próximo grupo.

\section{HbA1c inicio $>7 \%$ y $<9 \%$}

- En este grupo de pacientes se sugiere comenzar el tratamiento con terapia dual de inicio, la cual consiste en asociar metformina con iSGLT-2 o arGLP-1 con beneficio cardiovascular demostrado (Grado de recomendación I. Nivel de evidencia A).

- Si con la terapia dual inicial no se alcanzan los objetivos de $\mathrm{HbA} 1 \mathrm{c}$ en tres meses, se recomienda terapia triple, para lo cual la combinación sugerida es metformina + iSGLT-2 + arGLP-1 con beneficio cardiovascular demostrado (Grado de recomendación lla. Nivel de evidencia C).

- Ante la imposibilidad de utilizar fármacos con beneficio CV y/o mal control metabólico, asociar a metformina iDPP-4 (no utilizar combinado con arGLP-1), tiazolidinedionas (considerando riesgo de 
IC) y sulfonilureas (no usar glibenclamida) (Grado de recomendación Ila. Nivel de evidencia C).

\section{HbA1c inicio >9\%}

- En este grupo de pacientes el tratamiento depende de la presencia o no de síntomas. En caso de encontrarse el paciente sintomático, se recomienda inicio de tratamiento con insulina y evaluar posteriormente el agregado de fármacos con beneficio cardiovascular demostrado (Grado de recomendación I. Nivel de evidencia B).
- En ausencia de síntomas comenzar con terapia dual o triple terapia incluyendo fármacos con beneficio cardiovascular demostrado (Grado de recomendación I. Nivel de evidencia A).

Por último, se sugiere considerar en pacientes con terapia dual o triple (que no incluyan dentro de ella fármacos con beneficio cardiovascular demostrado) y adecuado control metabólico con enfermedad cardiovascular aterosclerótica, rotar a fármacos con beneficio cardiovascular demostrado: iSGLT-2 o arGLP-1.

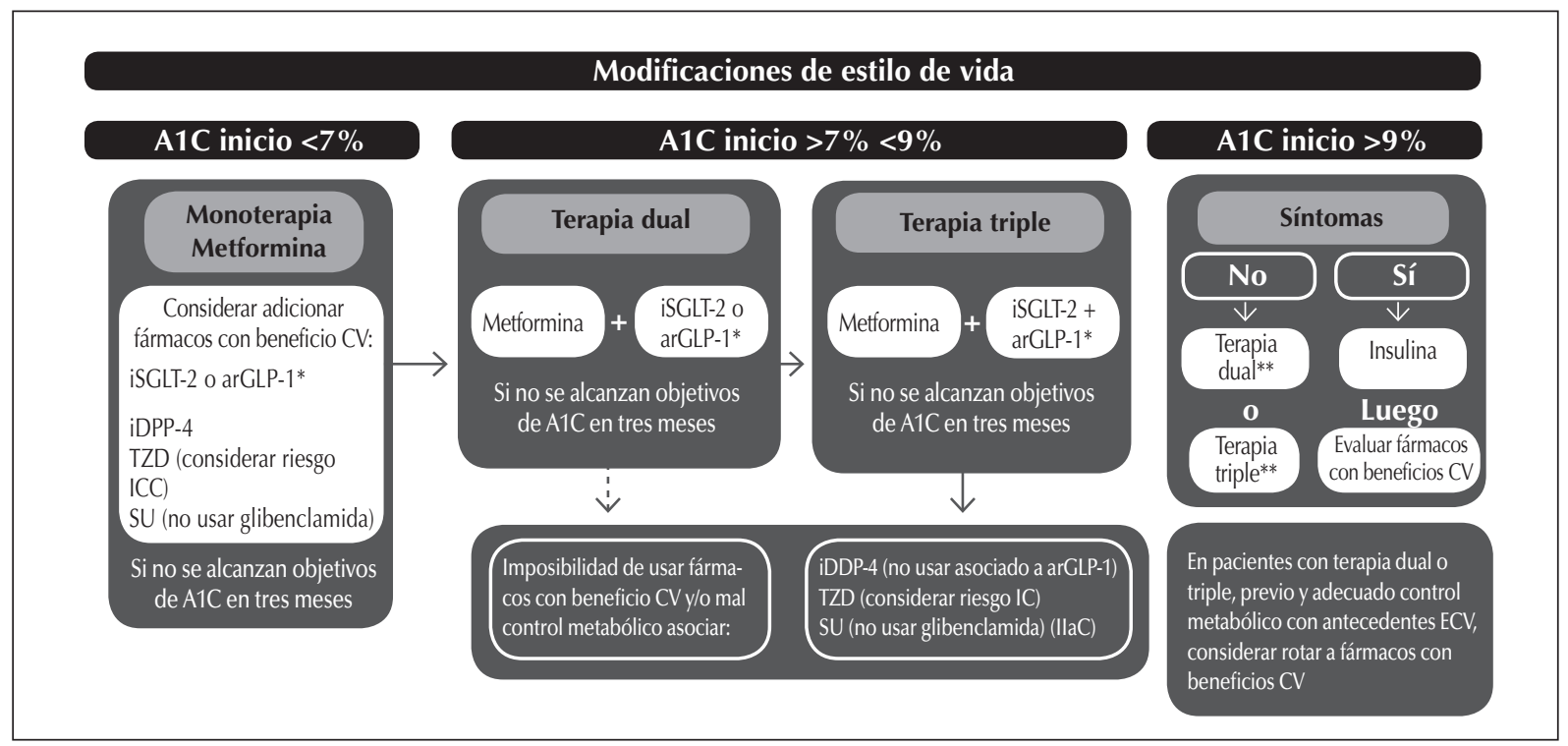

* Agonistas de receptores GLP-1 con beneficio cardiovascular; ${ }^{* *}$ Terapia dual: metformina + iSGLT-2 o arGLP-1; *** Terapia triple: metformina + iSLGLT-2 + arGLP-1.

Figura 5: Manejo de la hiperglucemia del paciente con DM2 y ECV aterosclerótica.

\section{Manejo de la hiperglucemia del paciente con DM2 con insuficiencia cardíaca con Fey $<\mathbf{4 0} \%$}

- En pacientes con insuficiencia cardíaca con fracción de eyección (Fey) $<40 \%$ (determinada por ecocardiografía) que se encuentren en clase funcional II-IV (NYHA), se indica comenzar tratamiento con cambios en el estilo de vida asociados a un iSGLT-2 (Grado de recomendación I. Nivel de evidencia B).

- En este grupo de pacientes se definieron tres grupos a partir de los niveles de $\mathrm{A} 1 \mathrm{C}$ iniciales: 1) $\mathrm{A} 1 \mathrm{C}$ inicio $<7,5 \%$; 2) $\mathrm{A} 1 \mathrm{C}$ inicio $>7,5 \%$ y $<9 \%$; 3) $\mathrm{A} 1 \mathrm{C}$ inicio $>9 \%$.

\section{HbA1c inicio $<7,5 \%$}

- En caso de no tolerar iSGLT-2 o encontrarse éstos contraindicados, se sugiere monoterapia con metformina (Grado de recomendación I. Nivel de evidencia C).
- De no poder utilizar metformina, se propone continuar monoterapia con arGLP-1 con beneficio CV demostrado, iDPP-4 (excepto saxagliptina) y sulfonilureas (excepto glibenclamida).

- En caso de no alcanzar los objetivos de A1C en tres meses, se sugiere terapia dual (véase inicio $\mathrm{A} 1 \mathrm{C}>7,5$ y $<9 \%$ ) (Grado de recomendación Ila. Nivel de evidencia B).

- Se contraindica el uso de glitazonas (Grado de recomendación III. Nivel de evidencia A).

\section{HbA1c inicio $>7,5 \%$ y $<9 \%$}

- Se indica iniciar tratamiento con terapia dual con iSGLT-2 asociados a metformina o arGLP-1 con beneficio cardiovascular demostrado (Grado de recomendación I. Nivel de evidencia A).

- Si no se logra el objetivo de $\mathrm{HbA1C}$ en tres meses, se sugiere terapia triple con iSGLT-2 aso- 
ciada a metformina + arGLP-1 con beneficio cardiovascular demostrado (Grado de recomendación Ila. Nivel de evidencia B).

- Ante la imposibilidad de utilizar fármacos con beneficio CV y/o mal control metabólico, asociar iDPP-4 (no combinar con arGLP-1 y no utilizar saxagliptina) o sulfonilureas (no utilizar glibenclamida) (Grado de recomendación lla. Nivel de evidencia B).

- Se contraindica el uso de glitazonas (Clase de recomendación III. Nivel de evidencia A).

\section{HbA1c inicio $>9 \%$}

- En este grupo de pacientes el tratamiento depende de la presencia o no de síntomas.

- En caso de encontrarse el paciente sintomático se recomienda inicio de tratamiento con insulina y evaluar posteriormente el agregado de iSGLT-2 (Grado de recomendación I. Nivel de evidencia B).

- En ausencia de síntomas comenzar con terapia dual o triple terapia, incluyendo iSGLT-2 (Grado de recomendación I. Nivel de evidencia A).

- Se contraindica el uso de glitazonas (Grado de recomendación III. Nivel de evidencia A).

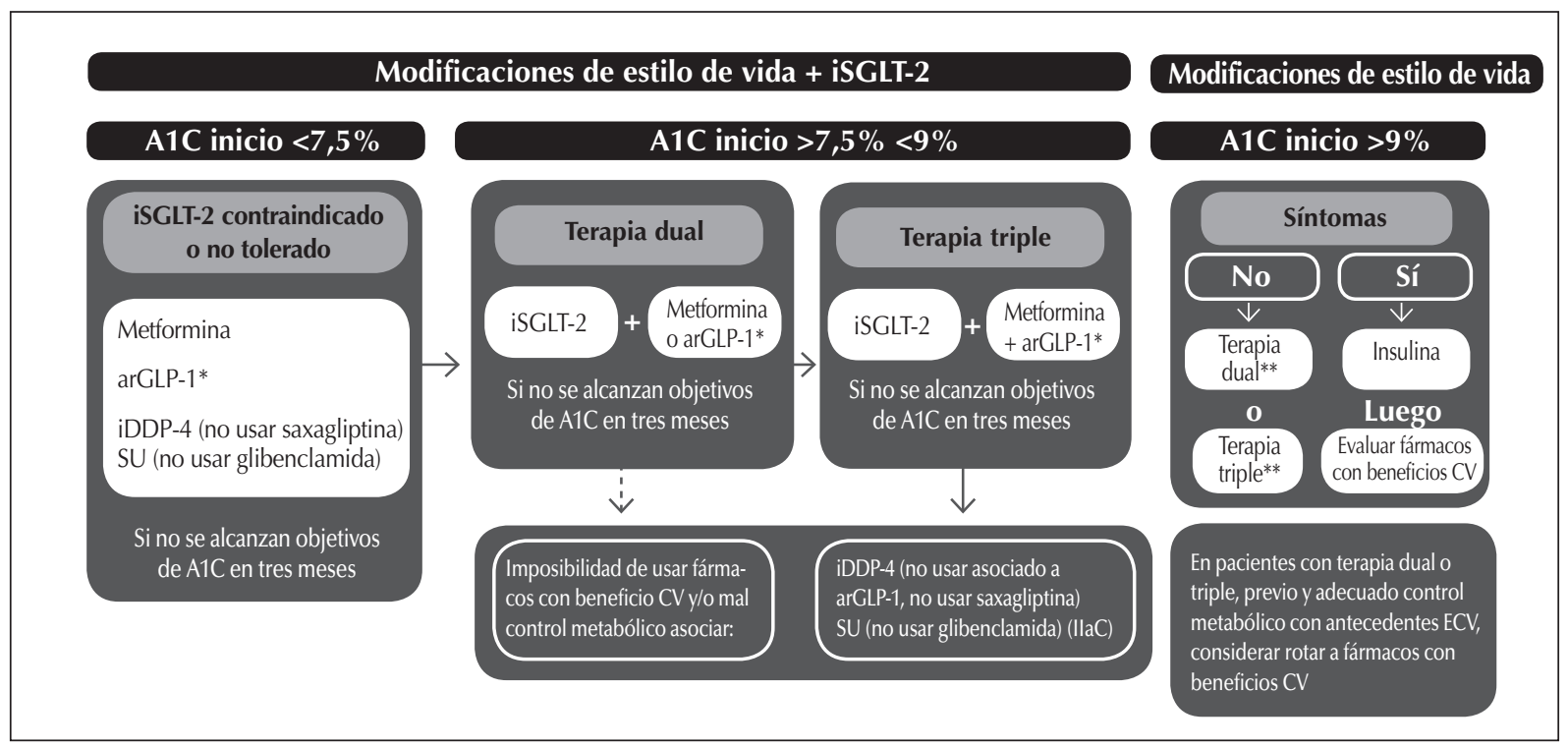

* Agonistas de receptores GLP-1 con beneficio cardiovascular; ** Terapia dual: iSGLT-2 + metformina o arGLP-1; *** Terapia triple: iSGLT-2 + metformina + ar GLP-1.

Figura 6: Manejo de la hiperglucemia del paciente con DM2 e insuficiencia cardíaca con Fey $<40 \%$.

\section{Manejo de la hiperglucemia del paciente con DM2 y riesgo cardiovascular alto}

Se definió como pacientes con DM y alto riesgo cardiovascular a aquellos que presentan un riesgo $>20 \%$ por puntaje (score) de la OMS o daño de órgano blanco o ateromatosis subclínica. En este caso, la primera línea de tratamiento consiste en modificaciones del estilo de vida, después de lo cual -partir de la A1C-se configuraron tres grupos de pacientes: 1) A1C inicio $<7,5 \%$; 2) A1C inicio $>7,5 \%$ y $<9 \%$; 3) A1C inicio $>9 \%$.

\section{A1C inicio $<7,5 \%$}

- Se sugiere iniciar tratamiento en monoterapia con metformina (Grado de recomendación I. Nivel de evidencia C).

- De no poder utilizar metformina, se sugiere considerar monoterapia con arGLP-1 o iSGLT-2 con beneficio CV demostrado (Grado de recomendación lla. Nivel de evidencia B); de no poder utilizar éstos, indicar iDPP-4, TZD y sulfonilureas (excepto glibenclamida).

- En caso de no alcanzar los objetivos de A1C en tres meses, se sugiere terapia dual (véase inicio $\mathrm{A} 1 \mathrm{C}>7,5$ y $<9 \%$ ). 
Revista de la Sociedad Argentina de Diabetes Año 55 Vol. 55 № 1 Suplemento Consenso Sociedad Argentina de CardiologíaSociedad Argentina de Diabetes Enero-abril de 2021: 03-70 ISSN 0325-5247 (impresa) ISSN 2346-9420 (en línea)

\section{A1C inicio $>7,5 \%$ y $<9 \%$}

- En este grupo de pacientes se sugiere iniciar tratamiento con terapia dual de entrada, la cual consiste en asociar metformina con iSGLT-2 o arGLP-1 con beneficio cardiovascular demostrado (Grado de recomendación I. Nivel de evidencia A).

- Si con la terapia dual inicial no se alcanzan los objetivos de $\mathrm{A} 1 \mathrm{C}$ en tres meses, se recomienda terapia triple, para lo cual la combinación sugerida es metformina + iSGLT-2 + arGLP-1 con beneficio cardiovascular demostrado (Grado de recomendación Ila. Nivel de evidencia C).

- Ante imposibilidad de utilizar fármacos con beneficio CV y/o mal control metabólico, asociar a metformina un iDPP-4 (no utilizar combinado con arGLP-1), tiazolindinedionas (considerando riesgo de IC) y sulfonilureas (no usar glibenclamida) (Grado de recomendación Ila. Nivel de evidencia C).

\section{A1C inicio $>9 \%$}

- En este grupo de pacientes el tratamiento depende de la presencia o no de síntomas.

- En caso de encontrarse el paciente sintomático se recomienda inicio de tratamiento con insulina y evaluar posteriormente el agregado de fármacos con beneficio cardiovascular demostrado (Grado de recomendación I. Nivel de evidencia B).

- En ausencia de síntomas comenzar con terapia dual o triple terapia incluyendo fármacos con beneficio cardiovascular demostrado (Grado de recomendación I. Nivel de evidencia A).

Por último, se sugiere considerar en pacientes con terapia dual o triple (que no incluyan dentro de ella fármacos con beneficio cardiovascular demostrado) y adecuado control metabólico con enfermedad cardiovascular aterosclerótica, rotar a fármacos con beneficio cardiovascular demostrado: iSGLT-2 o arGLP-1.

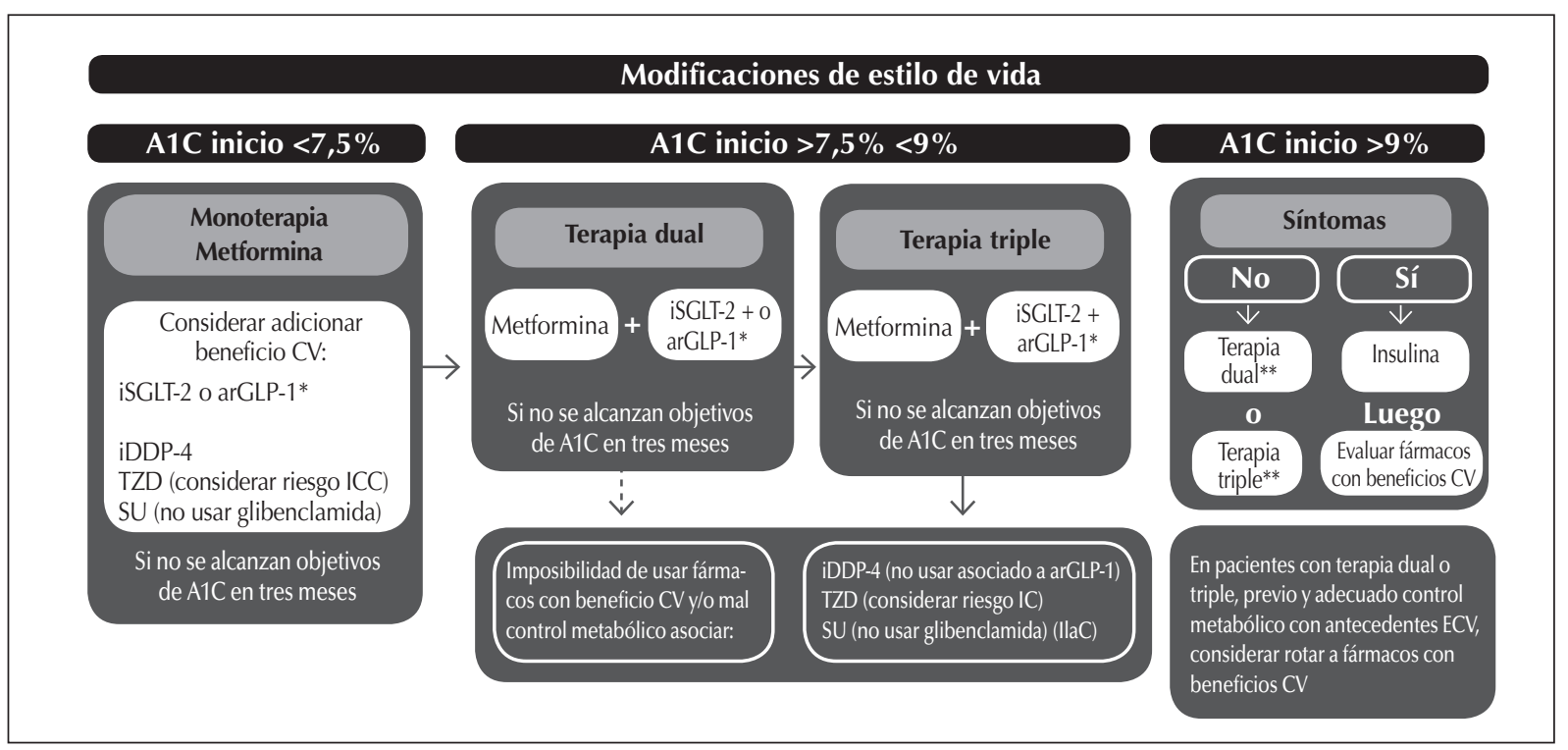

* Agonistas de receptor GLP-1 con beneficio cardiovascular; ** Terapia dual: metformina + iSGLT-2 o arGLP-1; *** Terapia triple: metformina + iSLGLT-2 + arGLP-1.

Figura 7: Manejo de la hiperglucemia del paciente con DM2 y alto riesgo cardiovascular.

\section{d. Cirugía metabólica en el paciente con DM}

A pesar del advenimiento de nuevas líneas de tratamiento farmacológico más eficientes, la evidencia del mundo real muestra que el control metabólico del paciente con DM dista de ser óptimo. Se entiende por cirugía metabólica toda intervención quirúrgica realizada en el tubo digestivo con el objeto de conseguir mejoría o remisión de la DM y reducción del riesgo cardiometabólico. Tradicionalmente se enfoca en relación con el IMC entre 30 y $35 \mathrm{~kg} / \mathrm{m}^{2}$, dado que para cifras mayores la indicación y el beneficio se expresan en relación con la cirugía bariátrica propiamente dicha. Dentro del subgrupo de pacientes que clasifica para ingresar en el programa quirúrgico de la DM, múltiples trabajos han 
mostrado beneficio en materia de control, si bien varios de ellos incluyen pacientes con DM con IMC mayor de $35 \mathrm{~kg} / \mathrm{m}^{2}$. De ellos se desprende que la tasa de remisión de la enfermedad observada oscila entre el 39 y el $90 \%$, permaneciendo en todos los casos por encima del grupo control destinado a tratamiento médico optimizado ${ }^{47}$. En el seguimiento a largo plazo, la tasa de remisión disminuye; sin embargo, el grupo cirugía mantiene la significancia estadística respecto del grupo tratamiento médico para el mejor control metabólico. En el grupo de pacientes sometidos a cirugía bariátrica se observan cambios significativos en el peso corporal, disminución del IMC, perímetro de cintura, descenso de triglicéridos, aumento de HDL, con información controvertida en materia de control de presión arterial y control de $\mathrm{LDL}^{48-50}$. De las múltiples técnicas quirúrgicas aprobadas en materia de cirugía bariátrica, cuando se circunscribe a pacientes con los criterios de IMC descriptos, y metabólicamente comprometidos, la evidencia señala que el bypass gástrico en Y de Roux (BPGYR) sería la técnica elegida, lográndose mejores resultados con respecto a la gastroplastia vertical (GV) o manga gástrica. En efecto, el BPGYR es significativamente más exitoso en la remisión de DM, pérdida de peso, control del perímetro de cintura, alcanzar HbA1c menor del $7 \%$ y control de LDL ${ }^{51}$. Otro aspecto promisorio de la cirugía metabólica es su capacidad de reducción del riesgo cardiovascular. Cuando se analiza una cohorte de pacientes con DM sometidos a cirugía metabólica, es posible advertir la caída estadísticamente significativa del riesgo cardiovascular calculado por UKPDS engine entre la situación prequirúrgica y al año del posoperatorio e independientemente del IMC de base ${ }^{52}$. Sin embargo, aún se aguardan los estudios prospectivos con seguimientos a 10 años que logren refrendar estas estimaciones calculadas. Para ser elegibles para el tratamiento quirúrgico, los pacientes deben cumplir los siguientes criterios básicos: tener diagnóstico de DM2 desde hace al menos dos años, ser menores de 65 años, mantener su hemoglobina glicosilada por encima del $8 \%$ por un año a pesar de tratamiento adecuado, IMC entre $30-34,9 \mathrm{~kg} / \mathrm{m}^{2}$, tener perímetro de cintura abdominal mayor de 102/88 cm (hombres/mujeres, respectivamente), con péptido $C$ en ayunas mayor de $1 \mathrm{ng} / \mathrm{dL}$, que demuestre compromiso y adherencia al tratamiento, que tenga posibilidad de seguimiento adecuado y que la indicación parta del especialista en enfermedades endocrino metabólicas con acuerdo del equipo quirúrgico tratante ${ }^{53}$. A su vez, entre los pacientes que cumplan con los criterios básicos se debe analizar la presencia de criterios mayores o menores para hacer la indicación formal de cirugía metabólica. La indicación de cirugía se recomienda para aquellos que reúnan al menos dos criterios mayores o uno mayor asociado con al menos dos menores (Cuadro 1).

\begin{tabular}{|l|}
\hline Criterios mayores \\
\hline Hipertensión arterial sostenida (TAS/TAD $>139 / 89 \mathrm{~mm} \mathrm{Hg}$ ) * \\
Dislipemia $(\mathrm{CT} / \mathrm{LDL} / \mathrm{TGL} / \mathrm{CnoHDL}>200 />100 />150 />100 \mathrm{mg} / \mathrm{dL}$ ) * \\
Antecedentes de enfermedad microvascular o macrovascular \\
SAHOS moderada a severa \\
\hline Criterios menores \\
\hline Insulinemia de ayuno $>20 \mu \mathrm{cU} / \mathrm{mL} \#$ \\
Espesor íntima-media $\geq 1 \mathrm{~mm}$ \\
Antecedentes heredofamiliares de enfermedad cardiovascular presente \\
Esteatosis hepática no alcohólica \\
\hline
\end{tabular}

*Con tratamiento o sin él; \#En pacientes no tratados con insulina. SAHOS: síndrome de apnea/hipopnea obstructiva del sueño.

Cuadro 1: Criterios mayores y menores para indicación de cirugía bariátrica

Resulta importante destacar que la cirugía metabólica es una herramienta más en el tratamiento de pacientes con DM. Considerando que la DM y la obesidad son enfermedades crónicas, se aconseja continuar en el posoperatorio con medicación tanto oral como inyectable para evitar recidivas y reganancia de peso.

\section{Recomendaciones}

- Se recomienda en pacientes con IMC igual a $40 \mathrm{~kg} / \mathrm{m}^{2}$ (IMC > 37,5 en asiáticos) o mayor, independientemente del nivel de control de glucemia o complejidad del tratamiento, y en pacientes con IMC entre 35-39,9 (IMC 32,5-37,4 kg/m² en asiáticos) con hiperglucemia inadecuadamente controlada a pesar de los cambios del estilo de vida y el tratamiento médico óptimo, asociado a dos criterios mayores o uno mayor y dos menores (Grado de recomendación Ila. Nivel de evidencia B).

- Considerada en pacientes con IMC 30-34, $9 \mathrm{~kg} / \mathrm{m}^{2}$ (27,5-32,4 en asiáticos) con hiperglucemia inadecuadamente controlada a pesar del tratamiento médico óptimo, ya sea por vía oral o inyectable, incluyendo insulina (Grado de recomendación Ila. Nivel de evidencia B). 
Revista de la Sociedad Argentina de Diabetes Año 55 Vol. 55 № 1 Suplemento Consenso Sociedad Argentina de CardiologíaSociedad Argentina de Diabetes Enero-abril de 2021: 03-70 ISSN 0325-5247 (impresa) ISSN 2346-9420 (en línea)

\section{BIBLIOGRAFÍA}

1. UK Prospective DM Study (UKPDS) Group. Intensive bloodglucose control with sulphonylureas or insulin compared with conventional treatment and risk of complications in patients with type 2 DM (UKPDS 33). Lancet 1998; 352:837-853.

2. Patel A, MacMahon S, Chalmers J, Neal B, Billot L, Woodward $\mathrm{M}$, Marre $\mathrm{M}$, et al. Intensive blood glucose control and vascular outcomes in patients with type 2 DM. N Engl J Med 2008; 358:2560-2572.

3. Ismail-Beigi F, Craven T, Banerji MA, Basile J, Calles J, Cohen $\mathrm{RM}$, et al. Effect of intensive treatment of hyperglycaemia on microvascular outcomes in type $2 \mathrm{DM}$ : an analysis of the ACCORD randomised trial. Lancet 2010; 376:419-430

4. Duckworth W, Abraira C, MoritzT, Reda D, Emanuele N, Reaven PD, et al. Glucose control and vascular complications in veterans with type 2 DM. N Engl J Med 2009; 360:129-139.

5. Einarson TR, Annabel A, Ludwig C, Panton U. Prevalence of cardiovascular disease in type 2 DM: a systematic literature review of scientific evidence from across the world in 2007-2017. Cardiovasc Diabetol 2018; 17:83-102.

6. Brady P,Terzic A. The sulfonylurea controversy: more questions from the heart. J Am Coll Cardiol 1998; 31:950-6.

7. Duncker D, Van Zon NS, Altman JD, Pavek TJ, Bache RJ. Role of $\mathrm{K}+\mathrm{ATP}$ channels in coronary vasodilation during exercise. Circulation 1993; 88:1245-53.

8. Garrat KN, Brady PA, Hassinger NL, Grill DE, Terzic A, Holmes DR Jr. Sulfonylurea drugs increase early mortality in patients with diabetes mellitus after direct angioplasty for acute myocardial infarction. J Am Coll Cardiol 1999; 33:119-24.

9. Colagiuri $S$, et al. The place of gliclazide MR in the evolving type 2 DM landscape: A comparison with other sulfonylureas and newer oral antihyperglycemic agents. DM Res Clin Pract 2018; 143:1-14.

10. Andersen S, Christensen M. Hypoglycaemia when adding sulphonylurea to metformin: a systematic review and network meta-analysis. Br J Clin Pharmacol 2016; 82:1291-1302.

11. Schopman JE, Simon AC, Hoefnagel SJ, Hoekstra JB, Scholten $\mathrm{RJ}$, Holleman F. The incidence of mild and severe hypoglycaemia in patients with type 2 diabetes mellitus treated with sulfonylureas: a systematic review and meta-analysis. DM Metab Res Rev 2014; 30:11-22.

12. Landman GW, de Bock GH, van Hateren KJ, van Dijk PR, Groenier KH, Gans RO, et al. Safety and efficacy of gliclazide as treatment for type 2 DM: a systematic review and meta-analysis of randomized trials. PLoS One 2014 Feb 12; 9(2):e82880.

13. Colagiuri S, Matthews D, Leiter LA, Chan SP, Sesti G, Marre M, et al. The place of gliclazide MR in the evolving type 2 DM landscape: A comparison with other sulfonylureas and newer oral antihyperglycemic agents. DM Res Clin Pract 2018; 143:1-14.

14. Simpson SH, Lee J, Choi S, Vandermeer B, Abdelmoneim AS, Featherstone TR, et al. Mortality risk among sulfonylureas: a systematic review and network meta-analysis. Lancet DM Endocrinol 2015; 3:43.

15. American Diabetes Association. 9. Pharmacologic approaches to glycemic treatment: Standards of Medical Care in Diabetes-2019. Diabetes Care 2019; 42(Suppl 1):S90-S102.

16. Rena G, Hardie DG, Pearson ER. The mechanisms of action of metformin. Diabetología 2017 Sep; 60(9):1577-1585.

17. Knowler WC, Barrett-Connor E, Fowler SE, Hamman RF, Lachin JM, Walker EA, et al. Reduction in the incidence of type 2 diabetes with lifestyle intervention or metformin. $\mathrm{N}$ Engl J Med 2002; 346:393-403.

18. Goldberg RB, Aroda VR, Bluemke DA, Barrett-Connor E, Budoff $\mathrm{M}$, Crandall JP, et al. Effect of long-term metformin and lifestyle in the DM Prevention Program and its outcome study on coronary artery calcium. Circulation 2017; 136(1):52-64.

19. Holman RR, Paul SK, Bethel MA, Matthews DR, Neil HA. 10year follow-up of intensive glucose control in type 2 diabetes. N Engl J Med. 2008; 359(15):1577-1589.
20. Aroda VR, Knowler WC, Crandall JP, Perreault L, Edelstein SL, Jeffries SL, el at. Metformin for DM prevention: insights gained from the DM Prevention Program/DM Prevention Program Outcomes Study. Diabetologia. 2017;60(9):1601-1611.

21. Libby P, Ridker PM, Hansson GK. Progress and challenges in translating the biology of atherosclerosis. Nature 2011; 473:317-25.

22. Nicholls SJ,Tuzcu EM, Wolski K, Bayturan O, Lavoie A, Uno K, et al. Lowering the triglyceride/ high-density lipoprotein cholesterol ratio is associated with the beneficial impact of pioglitazone on progression of coronary atherosclerosis in diabetic patients: insights from the PERISCOPE (Pioglitazone effect on regression of intravascular sonographic coronary obstruction prospective evaluation) study. J Am Coll Cardiol 2011; 57:153-9.

23. Nissen SE, Wolski K. Effect of rosiglitazone on the risk of myocardial infarction and death from cardiovascular causes. N Engl J Med 2007; 356:2457-71.

24. Home PD, Pocock SJ, Beck-Nielsen H, Gomis R, Hanefeld M, Jones NP, Komajda M, McMurray JJ. Rosiglitazone evaluated for cardiovascular outcomes -an interim analysis. N Engl J Med 2007; 357:28-38.

25. European Medicine Agency. Unión Europea; 2019. Acceso: 27 de Julio de 2019. Avandia. Disponible en: https://www.ema.europa. eu/en/medicines/human/EPAR/avandia.

26. Administración Nacional de Medicamentos, Alimentos y Tecnología médica [Internet]. Argentina: Farmacovigilancia; 2019. Acceso: 27 de Julio de 2019. Informe de farmacovigilancia. Disponible en: http://www.anmat.gov.ar/farmacovigilancia/informe_primer_semestre_2011.pdf.

27. Liao HW, Saver JL, WuYL, ChenTH, Lee M, Ovbiagele B. Pioglitazone and cardiovascular outcomes in patients with insulin resistance, pre-diabetes and type 2 diabetes: a systematic review and meta-analysis. BMJ Open. 20175; 7(1):e013927.

28. Sirica BM, Bhatt DL, Braunwald E, Steg PG, Davidson J, Hirshberg B, et al. Saxagliptin and cardiovascular outcomes in patients with type 2 diabetes mellitus. N Engl J Med 2013; 369:1317.

29. White WB, Cannon CP, Heller SR, Nissen SE, Bergnstal RM, Bakris GL, et al. Alogliptin after acute coronary syndrome in patients with type 2 DM. N Engl J Med 2013; 369:1327.

30. Green JB, Bethel MA, Armstrong PW, Buse JB, Engel SS, Garg $J$, et al. Effect of Sitagliptin on cardiovascular outcomes in type 2 DM. N Engl J Med 2015; 373:232.

31. Rosenstock J, Perkovic V, Johansen OE, Cooper ME, Kahn SE, Marx N, et al. Effect of linagliptin vs placebo on major cardiovascular events in adults with type $2 \mathrm{DM}$ and high cardiovascular and renal risk. The CARMELINA Randomized Clinical Trial. JAMA. 2019;321(1):69-79.

32. Pfeffer MA, Claggett B, Díaz R, Dickstein K, Gerstein HC, Kober $\mathrm{LV}$, et al. Lixisenatide in patients with type $2 \mathrm{DM}$ and acute coronary syndrome. N Engl J MED 2015; 373:2247-2257.

33. Marso SP, Daniels GH, Brown-Frandsen K, Kristensen P, Mann JFE, Nauck MA, et al. Liraglutide and cardiovascular outcomes in type 2 DM. N Engl J Med 2016; 375:311.

34. Marso SP, Bain SC, Consoli A, Elaschewitz FG, Jódar E, Leiter LA, et al. Semaglutide and cardiovascular outcomes in patients with type 2 DM. N Engl J Med 2016; 375:1834.

35. Hernández AF, Green JB, Janmohamed S, D'Agostino RB, Granger CB, Jones NP, et al. Albiglutide and cardiovascular outcomes in patients with type 2 DM and cardiovascular disease (Harmony Outcomes): a double-blind, randomised placebocontrolled trial. Lancet 2018; 392:1519.

36. Gerstein HC, Colhoun HM, Dagenais GR, Diaz R, Lakshmanan $M$, Pais $P$, et al. Dulaglutide and cardiovascular outcomes in type 2 DM (REWIND): a double-blind, randomised placebocontrolled trial. Lancet. 2019; 394:121-130.

37. Zelniker TA, Wiviott SD, Raz I, Im K, Goodrich El, Bonaca MP, et al. SGLT-2 inhibitors for primary and secondary prevention of cardiovascular and renal outcomes in type $2 \mathrm{DM}$ : a systematic review and metaanalysis of cardiovascular outcomes trials. Lancet 2019; 393:31-39 
38. Zinman B, Wanner C, Lachin JM, Fitchett D, Bluhmki E, Hantel $\mathrm{S}$, et Al. Empagliflozin, cardiovascular outcomes, and mortality in type 2 DM. N Engl J Med 2015; 373(22):2117-2128

39. Neal B, Perkovic V, Mahaffey KW, de Zeeuw D, Fulcher G, Erondu $\mathrm{N}$, et al. Canagliflozin and cardiovascular and renal events in type 2 DM. N Eng J Med 2017; 377:644.

40. Wiviott SD, Raz I, Bonaca MP, Mosenzon O, Kato ET, Cahn A, et al. Dapagliflozin and cardiovascular outcomes in type 2 DM. N Engl J Med 2019; 380:347-357

41. Perkovic V, Jardine MJ, Neal B, Bompoint S, Heerspink HJL, Charytan DM, et al. Canagliflozin and renal outcomes in type 2 DM and nephropathy. N Engl J Med 2019 13; 380(24):2295-230.

42. Fuente G, Sinay I, Costa Gil JE, Puchulu F, Dieuzeide G, Rodríguez M, Faingold MC, Litwak L. Insulinización en la diabetes mellitus tipo 2. Alternativas de intensificación. MEDICINA (Buenos Aires) 2016; 76: 173-179.

43. Haahr $\mathrm{H}$, Heise T. A review of the pharmacological properties of insulin degludec and their clinical relevance. Clin Pharmacokinet 2014; 53(9):787-800.

44. Gerstein HC, Bosch J, Dagenais GR, Díaz R, Jung H, Maggioni $A P$, et al. Basal insulin and cardiovascular and other outcomes in disglycemia. N Engl J Med 2012; 367:319-328.

45. Marso SP, McGuire DK, Zinman B, Poulter NR, Emerson SS, PieberTR, et al. Efficacy and safety of degludec versus glargine in type 2 diabetes. N Engl J Med 2017; 377(8):723-732.

46. LeeWJ, Aung L. Metabolic surgery for type 2 diabetes mellitus: Experience from Asia. Diabetes Metab J. 2016; 40(6):433-443.
47. International Hypoglycaemia Study Group. Hypoglycaemia, cardiovascular disease, and mortality in DM: epidemiology, pathogenesis, and management.Lancet DM Endocrinol 2019; 7: 385-96.

48. Schauer PR, Bhatt DL, Kirwan JP, Wolski K, Aminian A, Brethauer $S A$, et al. Bariatric surgery vs intensive medical therapy for diabetes. 5 year outcomes. N Engl J Med 2017; 376:641-51.

49. Ikramuddin S, Korner J, LeeWJ, Thomas AJ, Connett JE, Bantle $J P$, et al. Lifestyle intervention and medical management with vs without Roux-en-Y gastric bypass and control of hemoglobin A1c, LDL cholesterol, and systolic plood pressure at 5 years in the Diabetes Surgery Study. JAMA 2018; 319(3):266-278.

50. Hsu CC, Almulaifi A, Chen JC, Ser KH, Chen SC, Hsu KCJ, et al. Effect of bariatric surgery vs medical treatment on type 2 diabetes in patients with body mass index lower than 35: Five-Year Outcomes. JAMA Surg 2015 Dec; 150(12):1117-24.

51. Lee WJ, Keong C, Kong-Han S, Yi-Chih L, Shu-Chun C, JungChien $\mathrm{C}$, et al. Gastric bypass vs sleeve gastrectomy for type 2 diabetes mellitus: a randomized controlled trial. Arch Surg 2011; 146(2):143-148.

52. Wei JH, Chou RH, Huang PH, Lee WJ, Chen SC, Lin SJ. Metabolic surgery ameliorates cardiovascular risk in obese diabetic patients: Influence of different surgical procedures. Surg Obes Relat Dis 2018; 14(12):1832-1840.

53. Aguirre-Ackermann M, Alva O, Álvarez MG, Andreoni M, Babor E, Bottino V, et al. Consenso Argentino de Cirugía Metabólica. Revista de la Sociedad Argentina de Diabetes 2015; 49(3):95-110.

\section{Control de factores de riesgo}

\section{a. Hipertensión arterial}

La hipertensión arterial (HTA) es una comorbilidad frecuente en pacientes con DM, con una prevalencia que depende de varios factores entre los que se destacan: tipo de DM, edad, presencia de obesidad y etnia'. La prevalencia de HTA en pacientes con DM1 se aproxima al 30\% y, en pacientes con DM2, al 60\%. En pacientes con DM1, la presencia de HTA potencia la instalación y el empeoramiento de la nefropatía, mientras que, en pacientes con DM2, usualmente coexiste con otros factores de riesgo cardiometabólicos. Si bien no se dispone de recomendaciones absolutas en cuanto al uso del monitoreo ambulatorio de la presión arterial (MAPA) en los pacientes con $D M$, existe un consenso entre expertos en cuanto a su utilidad, particularmente para caracterizar el patrón nocturno de PA². El patrón "non dipper" es común en los pacientes con DM y es un fuerte predictor de eventos CV. Refleja alteraciones en la función autonómica, además de estar asociado al SAHOS, sobre todo en presencia de obesidad. Otro dato importante que aporta el MAPA consiste en la HTA sistólica durante el sueño, la cual puede predecir futuro desarrollo de microalbuminuria.
Por otro lado, el patrón de HTA oculta puede llegar a estar presente en el $50 \%$ de los pacientes con DM2. El tratamiento intensivo de la HTA en pacientes con DM reduce el riesgo de complicaciones macrovasculares y microvasculares. Se ha demostrado que reducir la PAS a valores $<140 \mathrm{mmHg}$ disminuye el riesgo CV. Por otro lado, la reducción a niveles de PAS $<130 \mathrm{mmHg}$ disminuye el riesgo de ACV y la progresión de retinopatía y albuminuria en grupos determinados (jóvenes con elevado riesgo de complicaciones con retinopatía, albuminuria y DM1), siempre que estos niveles de PA sean tolerados por el paciente ${ }^{3}$. Los resultados de los estudios ACCORD e Hipertension Optimal Treatment (HOT) avalan la recomendación de objetivo de PA $<140 / 90 \mathrm{mmHg}$ en pacientes con $\mathrm{DM}^{4,5}$. Los inhibidores del sistema renina-angiotensina son de particular valor, especialmente en pacientes con HTA y DM con alto riesgo CV, presencia de macroalbuminuria y microalbuminuria, y deben usare como fármacos de primera línea $a^{6,7}$. El tratamiento combinado de IECA con ARA II se asoció con un incremento de eventos adversos (hiperpotasemia, síncope y disfunción renal) ${ }^{8}$, por lo cual no 
Revista de la Sociedad Argentina de Diabetes Año 55 Vol. 55 № 1 Suplemento Consenso Sociedad Argentina de CardiologíaSociedad Argentina de Diabetes Enero-abril de 2021: 03-70 ISSN 0325-5247 (impresa) ISSN 2346-9420 (en línea)

está recomendada su utilización en forma conjunta. Existe evidencia que demuestra que la combinación de IECA con antagonistas cálcicos es superior a la de IECA con tiazidas. El objetivo principal del tratamiento es alcanzar la PA recomendada, para lo cual se indican como fármacos de primera línea los IECA o ARA II. Seguramente se requiera la combinación de otros fármacos a fin de alcanzar el objetivo terapéutico, para lo cual se aconseja la combinación con calcioantagonistas, diuréticos tiazídicos o símiles (indapamida); eventualmente, si fuera necesario o si existiera otra comorbilidad como arritmias, enfermedad coronaria o IC, podría agregarse un betabloqueante con perfil metabólico seguro.

\section{Recomendaciones}

- El control de la HTA en pacientes con DM disminuye el riesgo de eventos cardiovasculares (Grado de recomendación I. Nivel de evidencia A).

- Los pacientes con PA >120/80 mmHg deberían ser tratados con cambios en el estilo de vida (Grado de recomendación lla. Nivel de evidencia B).

- En pacientes con PA $>140 / 90 \mathrm{mmHg}$ se deberá adicionar, a los cambios en el estilo de vida, terapia farmacológica (Grado de recomendación I. Nivel de evidencia A).

- El objetivo de PA con el tratamiento es $<140 / 90 \mathrm{mmHg}$ (Grado de recomendación I. Nivel de evidencia $\mathrm{C}$ ).

- La PAS < 130/80 mmHg puede ser apropiada en ciertos pacientes con DM (jóvenes con albuminuria, más un factor de riesgo cardiovascular), si es bien tolerada por ellos (Grado de recomendación I. Nivel de evidencia C).

- La terapia no farmacológica consiste en descenso del peso corporal si el paciente tiene sobrepeso/obesidad, Dietary Approaches to Stop Hypertension (DASH) y actividad física (Grado de recomendación I. Nivel de evidencia B).

- La terapia farmacológica debe incluir IECA o ARA II pero no ambos fármacos combinados (Grado de recomendación I. Nivel de evidencia B).

- Generalmente se requieren dos o más fármacos para el control de la HTA en el paciente con DM, como IECA o ARA II, antagonistas cálcicos o tiazidas en dosis máximas. Deberán monitorearse la función renal y los niveles de potasio (Grado de recomendación I. Nivel de evidencia B).

- En DM pregestacional con HTA crónica, el objetivo de tratamiento es 129-110/79-65 mmHg (Grado de recomendación Ilb. Nivel de evidencia C).

\section{b. Dislipidemia}

Las discusiones correspondientes a la fisiopatología de la dislipidemia en DM y al abordaje del estudio lipídico se encuentran desarrolladas en el Anexo I.

\section{Tratamiento farmacológico para prevención CV} Estatinas

La presencia de DM2 duplica la mortalidad CV en comparación con los sujetos sin $\mathrm{DM}^{9}$. Algunos estudios, aunque no todos, mostraron que el pronóstico CV de los pacientes con DM sin infarto agudo de miocardio era similar al de los sujetos sin DM, pero con antecedentes de infarto agudo de miocardio ${ }^{10-12}$. La importancia de "categorizar" a los sujetos con DM con un riesgo más elevado se traduce en impulsar medidas preventivas más enérgicas. En ese contexto, las estatinas constituyen herramientas terapéuticas de primera línea. Dependiendo del tipo y dosis utilizada, la reducción del C-LDL en los pacientes diabéticos oscila entre un 24 y $52 \%{ }^{13,14}$. La mayoría de los ensayos clínicos que evaluó el impacto de las estatinas en la incidencia de eventos macrovasculares, tanto en prevención primaria como secundaria, no se realizaron específicamente en sujetos con DM. De hecho, la proporción de pacientes con DM representa entre el 1 y el 35\% de la población total según el estudio analizado ${ }^{15}$. Una excepción fue el Collaborative Atorvastatin Diabetes Study (CARDS) ${ }^{16}$, que aleatorizó a 1.410 pacientes con DM y 1.428 controles a recibir atorvastatina $10 \mathrm{mg} /$ día vs placebo. El estudio finalizó tempranamente (3,9 años) debido a una reducción significativa de los eventos CV en los sujetos que recibieron estatinas: disminución de riesgo de eventos coronarios del 36\% (IC 95\%: 9-55\%) y del 48\% (IC 95\%: 11-69\%) el de ACV. Otros dos estudios también evaluaron el impacto de las estatinas exclusivamente en una población de pacientes con DM. El Deutsche Diabetes Dialyse Studie (4D trial) evaluó sólo pacientes en hemodiálisis, pero no mostró una reducción significativa de eventos en este subgrupo particular de pacientes con DM ${ }^{17}$. Asimismo, el estudio Atorvastatin Study for Prevention of Coronary Heart Disease Endpoints in Non-Insulin-Dependent Diabetes Mellitus (ASPEN), con algunos cuestionamientos metodológicos y seleccionando una subpoblación de bajo riesgo, tampoco demostró una diferencia estadísticamente significativa en la reducción de los eventos $\mathrm{CV}$ a favor de las estatinas ${ }^{18}$. Dos subanálisis de grandes ensayos también analizaron 
el impacto de las estatinas de moderada intensidad en los pacientes con DM. En el estudio Heart Protection Study (HPS), la utilización de simvastatina 40 mg/día en comparación con el placebo se asoció con una reducción (primer evento $\mathrm{CV}$ mayor) del 25\% (IC 95\%: 13-30\%) en toda la población con DM ( $n=5.963)$ y del 33\% (IC 95\%: 17-46\%) en los pacientes con DM sin enfermedad $\mathrm{CV}$ al inicio del estudio $(n=2.912)^{19}$. Asimismo, en un subanálisis del estudio Anglo-Scandinavian Cardiac Outcomes TrialLipid Lowering Arm (ASCOT-LLA), que incluyó 2.532 pacientes diabéticos sin antecedentes $\mathrm{CV}$, el uso de atorvastatina $10 \mathrm{mg}$ en comparación con el placebo se asoció con una reducción significativa del 23\% (IC 95\%: 2-39\%) en el punto final combinado de eventos vasculares ${ }^{20}$. Finalmente, un metaanálisis que incluyó sólo pacientes con DM (14 ensayos clínicos aleatorizados, 18.686 pacientes) demostró que la terapia con estatinas redujo $9 \%$ la mortalidad de cualquier causa y un $21 \%$ los eventos CV mayores por cada $1 \mathrm{mmol} / \mathrm{L} 939 \mathrm{mg} / \mathrm{dL}$ de reducción del C-LDL ${ }^{15}$. Dichos hallazgos se observaron independientemente de las características basales de la población, incluyendo el antecedente de enfermedad CV y el nivel de C-LDL. La evidencia evaluada hasta ahora, en su gran mayoría, analizó el tratamiento con estatinas de moderada intensidad. A la fecha, no se cuenta con ensayos clínicos que hayan evaluado tratamiento con estatinas en alta intensidad sólo en una población de pacientes con DM. Sin embargo, un análisis post hoc del ensayo Study of Coronary Atheroma by Intravascular Ultrasound: Effect of Rosuvastatin versus Atorvastatin (SATURN) reveló que las estatinas en alta intensidad producen regresión de la aterosclerosis (estimado por ultrasonido coronario) en pacientes con DM, de forma similar a los sujetos sin DM, sólo cuando se alcanzan valores muy bajos de C-LDL $(<70 \mathrm{mg} / \mathrm{dL})^{22}$. Asimismo, en el análisis de subgrupos del metaanálisis que comparó estatinas en alta intensidad vs moderada intensidad, se evidenció el mismo beneficio en la población con DM o sin ella. Esto último tiene relevancia clínica y refuerza la necesidad de intensificar el tratamiento hipolipemiante y buscar una mayor reducción del C-LDL en los pacientes con DM que presenten un perfil de riesgo más elevado ${ }^{23}$.

\section{Ezetimibe}

El ezetimibe interactúa con el transportador intestinal de esteroles Niemann-Pick C1-like 1 (NPC1L1) inhibiendo selectivamente la absorción intestinal del colesterol biliar y dietario. Mediante este mecanismo reduce la carga de colesterol en los quilomicrones (QM), induce una mayor expresión de receptores hepáticos de C-LDL y disminuye los niveles plasmáticos de C-LDL ${ }^{24,25}$. Un análisis agrupado que incluyó más de 21.000 sujetos demostró una reducción promedio ajustada del C-LDL de $23 \%$ a favor del tratamiento combinado con respecto a la monoterapia con estatinas. Sin embargo, la respuesta hipolipemiante del ezetimibe es heterogénea y la variabilidad interindividual marcada ${ }^{26}$. Ciertos pacientes son hiperrespondedores y logran reducciones del C-LDL sustancialmente mayores que el descenso promedio habitual ${ }^{27}$. Un estudio de cinética de lipoproteínas demostró que los pacientes con DM2 y enfermedad coronaria tienen aumentada la absorción intestinal de colesterol. Varios estudios genéticos efectuados más recientemente encontraron una mayor expresión del ARNm del NPC1L1 y menor expresión del ARNm de los transportadores ABCG5 y ABCG8 que expulsan colesterol desde el enterocito a la luz intestinal. Se podría concluir que los pacientes con DM2 tienen sobrerregulada la absorción intestinal de colesterol28-31. Estos hallazgos fundamentan la mayor reducción de C-LDL, C-no HDL y apolipoproteína B que se observó al asociar estatinas con ezetimbe en pacientes con DM2 cuando se comparó la respuesta con la de pacientes sin $\mathrm{DM}^{32}$. El estudio Stop Atherosclerosis in Native Diabetics Study (SANDS) demostró que la reducción intensiva del C-LDL $(<70 \mathrm{mg} / \mathrm{dL})$, en individuos con DM2, producía una regresión del espesor íntima-media carotídea similar, independientemente del esquema hipolipemiante utilizado (estatinas o estatinas + ezetimibe) ${ }^{33}$.

Hasta el momento no se diseñó un estudio clínico específico con el objetivo de evaluar el beneficio adicional del agregado de ezetimibe al tratamiento con estatinas en pacientes con DM2. Dos ensayos clínicos que incluyeron un apreciable número de pacientes con DM2 pueden ayudarnos a responder este interrogante, a pesar de las limitaciones metodológicas que tienen los análisis de subgrupos. El Study of Heart and Renal Protection (SHARP) fue un estudio aleatorizado doble ciego que incluyó 9.270 pacientes con IRC (2.094 DM2), que evaluó el beneficio clínico de la reducción intensiva del C-LDL con ezetimibe $10 \mathrm{mg}$ y simvastatina $20 \mathrm{mg}$ comparado con placebo. La reducción proporcional y absoluta del riesgo fue 
numéricamente mayor en la subpoblación de pacientes con DM2 (22\% vs $14 \%$ y $4,2 \%$ vs $1.5 \%$, respectivamente), sin embargo, no se demostró heterogeneidad entre subgrupos ${ }^{34}$. El estudio IMProved Reduction of Outcomes: Vytorin Efficacy International Trial (IMPROVE-IT) incluyó 18.144 pacientes (4.933 DM2) hasta los 10 días posteriores de haber sufrido un síndrome coronario agudo, aleatorizados a recibir $40 \mathrm{mg}$ de simvastatina/placebo o $40 \mathrm{mg}$ de simvastatina combinada con $10 \mathrm{mg}$ de ezetimibe con un seguimiento de siete años. Los pacientes con DM2 tratados con ezetimibe y simvastatina tuvieron menor riesgo de sufrir un IAM (HR 0,76; IC 95\%: 0,66-0,88), un ACV isquémico (HR 0,61; IC 95\%: 0,46-0,82), o el punto final combinado de muerte CV, IAM o ACV (HR 0,80; IC 95\%: 0,71-0,90) al compararlos con pacientes sin DM ( $P$ de interacción: 0,028, 0,031 y 0,016 , respectivamente) ${ }^{35}$. Por último, en un subanálisis del IMPROVE-IT, se observó que los pacientes con DM2 y enfermedad polivascular constituyen un fenotipo vascular maligno con un riesgo de eventos cercano al 10\% año. En este subgrupo, la reducción de riesgo absoluto fue mayor y el número necesario de tratar de sólo 11 pacientes $^{36}$. El IMPROVE-IT demostró la seguridad del ezetimibe en pacientes con DM2, sin constatarse incremento en la tasa de eventos adversos musculares, hepáticos, vesiculares ni cáncer con respecto al placebo. Sólo se observó un leve aumento en la incidencia de ACV hemorrágico $(0,9 \% \text { vs } 0,4 \%)^{27,34}$. En conclusión, diversos estudios (genéticos, fisiopatológicos, metabólicos y de beneficio clínico) posicionan al ezetimibe como el tratamiento hipolipemiante suplementario de elección en pacientes con DM2 que no alcanzan la meta de C-LDL con estatinas. En algunos pacientes con DM2 y enfermedad vascular, dislipidemia combinada, niveles elevados de C-no HDL y/o apolipoproteína $B$, podría considerarse como terapia combinada desde el inicio del tratamiento.

\section{Fibratos}

Los fibratos son fármacos que ejercen su función básica disminuyendo los niveles de TG. La única indicación aceptada para la utilización de fibratos como compuesto no asociado a estatinas es en casos de hipertrigliceridemia severa (mayor de $500 \mathrm{mg} / \mathrm{dl}$ ) para prevenir pancreatitis aguda ${ }^{37}$. Algunos autores proponen un punto de corte mayor, por encima de $885 \mathrm{mg} / \mathrm{dl}$, como indicación de fibratos para prevención de pancreatitis ${ }^{38}$. La combinación de fibratos y estatinas se ha evaluado en población de pacientes con DM en el estudio ACCORD, sin demostrarse un beneficio en prevención de eventos $C V$. Sin embargo, el análisis de subgrupo preespecificado demostró beneficio clínico en aquellos pacientes con niveles de TG mayores desde $204 \mathrm{mg} / \mathrm{dL}$ y de C-HDL menores de $34 \mathrm{mg} / \mathrm{dL}^{39}$. El estudio Fenofibrate Intervention and Event Lowering in Diabetes (FIELD), en un análisis de subgrupo similar, evidenció los mismos beneficios, agregando clara mejoría en cuanto a la microangiopatía (retinopatía, neuropatía y nefropatía $)^{40}$. Cabe mencionar que la combinación de estatinas y fibratos conlleva un riesgo aumentado de miopatía y elevación de transaminasas, estando contraindicado el gemfibrozil en combinación con estatinas (no así el fenofibrato) ${ }^{41}$. Por último, el estudio Pemafibrate to Reduce Cardiovascular OutcoMes by Reducing Triglycerides IN patiENts With diabeTes (PROMINENT) con pemafibrato se encuentra en curso, evaluando pacientes con DM bajo tratamiento con estatinas y niveles elevados de TG y bajos de C-HDL. Este ensayo brindará valiosa información con respecto al lugar de los fibratos en el manejo de la dislipidemia en la población de pacientes con $\mathrm{DM}^{42}$. En conclusión, podemos afirmar que el agregado de fibratos a estatinas, en pacientes con DM, hasta la fecha no ha demostrado evidencia de beneficio clínico en cuanto a la prevención de enfermedad CV aterosclerótica, por lo cual no se puede recomendar para tal fin. El agregado de estos fármacos podría considerarse en pacientes diabéticos que combinen hipertrigliceridemia con C-HDL bajo ${ }^{43}$.

\section{Ácidos grasos poliinsaturados omega-3}

Los ácidos grasos poliinsaturados omega-3 son ácidos grasos esenciales que se incorporan con la dieta. En este grupo se encuentran principalmente los ácidos alfa-linolénico ( $A L A)$, eicosapentaenoico (EPA) y docosahexaenoico (DHA). Sus principales fuentes naturales son los alimentos marinos (pescado, mariscos y microalgas), que contienen EPA y DHA preformado, las formas bioactivas de los AG n-3.

Desde el punto de vista $C V$, una de las características más importantes de los AG n-3 son sus propiedades antiinflamatorias. EPA y DHA antagonizan los derivados inflamatorios del ácido araquidónico, regulan la expresión génica de procesos 
inflamatorios, como la adhesión celular y la secreción de interleuquinas, estabilizan la placa de ateroma y causan vasodilatación arterial. A través de la disminución de los niveles de apo C-III, reducen los niveles de TG y de partículas aterogénicas, como las LDL pequeñas y densas, y los $\mathrm{OM}$ remanentes ${ }^{44}$. Algunos estudios observacionales relacionaron el consumo de pescado con menores tasas de enfermedad $\mathrm{CV}^{45,46}$. Sin embargo, estos resultados no han sido reproducidos en los ensayos clínicos con suplementación de AG n-3. Dos grandes metaanálisis recientes no evidenciaron beneficios cardiovasculares significativos en ningún punto final, ni siquiera en subgrupos de mayor riesgo como pacientes con DM o con enfermedad CV establecida, con la administración de AG n-3 37,48 .

En el estudio Effects of Aspirin for Primary Prevention in Persons with Diabetes Mellitus (ASCEND) se aleatorizaron a 15.000 pacientes con DM sin enfermedad CV establecida, en dos grupos: uno recibió AG n-3 (460 mg EPA + 380 mg DHA) y el otro grupo placebo. En consonancia con la evidencia previa, no se detectaron diferencias significativas en eventos cardiovasculares durante los 7,4 años de seguimiento promedio ${ }^{49}$. El estudio Reduction of Cardiovascular Events With Icosapent Ethyl-Intervention (REDUCE-IT) se diferencia de los demás, ya que evaluó la suplementación de altas dosis de AG n-3 (4 g de EPA) vs placebo en 8.000 pacientes de alto riesgo $\mathrm{CV}$ (pacientes con DM, más otros factores de riesgo, o pacientes en prevención secundaria), sumado al tratamiento con estatinas eventualmente asociadas a ezetimibe. En los 4,9 años de seguimiento promedio se evidenció una reducción del $25 \%$ en el punto final combinado de muerte $\mathrm{CV}$, infarto agudo de miocardio, accidente cerebrovascular, revascularización y angina inestable. Analizando los eventos por separado, hubo reducción significativa de mortalidad CV (20\%), IAM (31\%) y ACV (28\%), e incluso una reducción de mortalidad por todas las causas (13\%), pero que marginalmente no alcanzó significancia estadística. En este estudio, un 58\% de los pacientes presentaba DM y el beneficio se observó tanto en aquellos con DM (RRR 23\% del punto final combinado) como en sin DM (RRR 27\%). Como efectos adversos, se observó un pequeño pero significativo aumento en la incidencia de fibrilación auricular y de sangrados no fatales ${ }^{50}$. El estudio REDUCE-IT es el primero en demostrar reducción de eventos vasculares en pacientes con hipertrigliceridemia bajo tratamiento con estatinas utilizando altas dosis de EPA purificado.

\section{Inhibidores de PCSK9}

La proproteína convertasa subtilisina kexina tipo 9 (PCSK9) es reguladora del metabolismo lipídico. Su mecanismo de acción radica en su interacción con el receptor de LDL. La PCSK9 circulante se une al rLDL en la membrana de los hepatocitos. Esto modifica el destino del rLDL de su vía normal de reciclado a la superficie de la célula y, en su lugar, lo dirige a degradación lisosomal ${ }^{51}$. Por lo tanto, a mayores niveles de PCSK9 circulante, mayor degradación del rLDL, lo cual reduce el número de dichos receptores de superficie en el hepatocito aumentando los niveles plasmáticos de C-LDL ${ }^{52}$. La utilización de anticuerpos monoclonales totalmente humanizados contra PCSK9, capaces de interferir con la unión de esta proteína y el rLDL, ha demostrado ser un efectivo mecanismo hipolipemiante ${ }^{53}$. Los iPCSK9 generan una reducción del C-LDL del 55\%, del colesterol total del 36\%, del C-no HDL del $52 \%$, de la apolipoproteína B del $46 \%$, de la lipoproteína (a) del 30\% y de los TG del $16 \%$. Se informa también un aumento del C-HDL del 7\% y de la apolipoproteína A-I del 6\% ${ }^{54,55}$. Por otro lado, cuando se asociaron con estatinas, los iPCSK9 alcanzaron una disminución de C-LDL del $54-74 \%$ comparados con placebo, y del 26 a $46 \%$ comparados con ezetimibe ${ }^{56}$. El estudio Further Cardiovascular Outcomes Research with PCSKO Inhibition in Subjects with Elevated Risk (FOURIER) fue el primer ensayo clínico en demostrar reducción de eventos CV con la aplicación de un iPCSK9 ${ }^{57}$. Este estudio incluyó 27.564 pacientes con enfermedad CV aterosclerótica y niveles de C-LDL de más de $70 \mathrm{mg} / \mathrm{dL}$ a pesar del uso de estatinas de alta o moderada intensidad. Fueron asignados al azar a recibir evolocumab (140 mg cada dos semanas o $420 \mathrm{mg}$ mensuales) o placebo. A las 48 semanas, los participantes del estudio con evolocumab habían logrado una media de C-LDL de $30 \mathrm{mg} / \mathrm{dL}$, con $42 \%$ de los pacientes alcanzando un C-LDL de menos de $25 \mathrm{mg} / \mathrm{dL}$. Esto se tradujo en una RRR del 15\% (IC 95\% 8-21\%; $p<0,001$ ) para el punto final compuesto primario (muerte $C V$, IAM, ACV, internación por angina inestable o revascularización coronaria) y el 20\% (IC 95\%: $12-27 \% ; p<0,001)$ para el punto final combinado secundario (muerte CV, IAM, ACV). La magnitud del beneficio con evolocumab en el estudio FOU- 
RIER basado en el descenso de C-LDL por $\mathrm{mg} / \mathrm{dL}$ es, en gran medida, consistente con los beneficios cardiovasculares documentados en el análisis combinado de ensayos anteriores con estatinas. Por lo tanto, los resultados del ensayo FOURIER refuerzan y amplían la hipótesis "cuanto más bajo, mejor" al demostrar los beneficios CV de disminuir el C-LDL a niveles inferiores a los de cualquier estudio previo. En el mismo sentido, el estudio Evaluation of Cardiovascular Outcomes After an Acute Coronary Syndrome During Treatment With Alirocumab (ODYSSEY OUTCOMES) aleatorizó la utilización de alirocumab 75 mg cada dos semanas contra placebo, en una población de 18.924 pacientes dentro del año de haber sufrido un evento coronario agudo y con niveles de C-LDL mayores de $70 \mathrm{mg} / \mathrm{dL}^{58}$. Alirocumab redujo la incidencia del punto final combinado primario $(9,5 \%$ vs $11,1 \%$, HR: 0,85; IC 95\%: 0,78-0,93; $p<0,001)$.
Un aspecto interesante de este estudio es que alirocumab demostró mayor eficacia en aquellos pacientes con un C-LDL basal mayor de $100 \mathrm{mg} /$ dL. Se observó que el $28,8 \%$ de los pacientes del estudio padecía DM2, los cuales mostraron, en un análisis preespecificado de subgrupos, que presentaban el doble de beneficio comparados con aquellos sin $\mathrm{DM}^{59}$. Coincidentemente, un análisis post hoc de los estudios ODYSSEY observó que aquellos pacientes pertenecientes a grupos de alto riesgo (por ejemplo, la DM) tenían mayor beneficio del agregado de alirocumab ${ }^{60}$. Dado el costo de estos fármacos, algunos documentos postulan que, a pesar de su probada eficacia clínica, su costo-efectividad es discutida ${ }^{61}$. Por este motivo deben adaptarse las indicaciones de estos fármacos a las dificultades de acceso y costo de cada paciente, tratando de reservarlas para aquellos de mayor riesgo.

\begin{tabular}{|l|l|l|}
\hline & Objetivo C-LDL ideal & Objetivo de C-no HDL \\
\hline DM sin otros factores de riesgo CV o daño de órgano blanco & $<100 \mathrm{mg} / \mathrm{dL}$ o reducción del $50 \%$ & $<130 \mathrm{mg} / \mathrm{dL}$ \\
\hline DM con factores de riesgo CV o daño de órgano blanco & $<70 \mathrm{mg} / \mathrm{dL}$ o reducción del $50 \%$ & $<130 \mathrm{mg} / \mathrm{dL}$ \\
\hline DM + enfermedad CV & $<70 \mathrm{mg} / \mathrm{dL}$ o reducción del $50 \%$. Razonable $<55 \mathrm{mg} / \mathrm{dL}$ & $<130 \mathrm{mg} / \mathrm{dL}$ \\
\hline
\end{tabular}

DM: diabetes mellitus; CV: cardiovascular.

Tabla 1: Objetivos terapéuticos de LDL colesterol.

\section{Recomendaciones}

- Se recomienda utilizar estatinas como fármacos hipolipemiantes de primera línea en los pacientes con DM para lograr el objetivo terapéutico de C-LDL (Grado de recomendación I. Nivel de evidencia A).

- Debe agregarse ezetimibe como herramienta complementaria a las estatinas en caso de no alcanzarse el objetivo terapéutico en pacientes con DM y enfermedad cardiovascular establecida (Grado de recomendación I. Nivel de evidencia A).

- El agregado de ezetimibe asociado a las estatinas podría ser beneficioso para alcanzar el objetivo terapéutico en pacientes con DM en prevención primaria (Grado de recomendación lla. Nivel de evidencia C).

- En los pacientes con DM y enfermedad cardiovascular establecida debe considerarse el agregado de un iPCSK9 cuando no se ha logrado el objetivo terapéutico, con la máxima dosis tolerada de estatina y el agregado de ezetimibe, especialmente cuando los niveles de cLDL son $>100 \mathrm{mg} / \mathrm{dL}$ (Grado de recomendación I. Nivel de evidencia A).

- Se recomienda el uso de fibratos en pacientes con hipertrigliceridemia mayor de $500 \mathrm{mg} / \mathrm{dL}$ para reducir el riesgo de pancreatitis. El tratamiento deberá instaurarse de inmediato cuando los valores de triglicéridos superen los 885 mg/dL (Grado de recomendación I. Nivel de evidencia C).

- La adición de fibratos al tratamiento con estatinas debe considerarse en pacientes con DM que persistan con $\mathrm{HDL}<35 \mathrm{mg} / \mathrm{dL}$ y triglicéridos $>200 \mathrm{mg} / \mathrm{dL}$ (Grado de recomendación Ilb. Nivel de evidencia B).

- No se recomienda el uso sistemático de ácidos grasos omega-3 en todos los pacientes con DM (Grado de recomendación III. Nivel de evidencia A).

- El agregado de altas dosis de omega-3 éster de eicosapentil purificado podría usarse para la reducción del riesgo CV en pacientes con DM e hipertrigliceridemia leve a moderada (Grado de recomendación Ilb. Nivel de evidencia B).

\section{c. Antiagregación plaquetaria}

La activación plaquetaria cumple un papel fundamental en el proceso aterosclerótico y aterotrombó- 
tico. Es por ello que su inhibición ha sido objeto de estudio en numerosas poblaciones. En la actualidad la función de los antiagregantes (en especial la aspirina) en prevención primaria está en profunda revisión. En muchos países se recomienda no utilizarla ${ }^{62}$, incluso en pacientes con DM sin enfermedad CV.

\section{Ácido acetilsalicílico (AAS)}

EI AAS inhibe en forma irreversible la enzima ciclooxigenasa 1 (COX-1) a través de la acetilación del grupo hidroxilo del residuo de serina. Su utilización ha sido durante años la piedra angular del tratamiento antiagregante. Actualmente, la utilidad e indicación en prevención secundaria, independientemente de la presencia de DM, es clara. Sin embargo, su empleo en prevención primaria es un poco más discutido, en parte debido a que conlleva un aumento del riesgo hemorrágico principalmente a nivel gastrointestinal. Muchos de los estudios realizados para evaluar el efecto del AAS en pacientes con DM sin evento cardiovascular previo corresponden a poblaciones japonesas (Japanese Primary Prevention of Atherosclerosis With Aspirin for Diabetes/Prevention of Progression of Arterial Disease and Diabetes; JPAD/POPADAD) ${ }^{63-64}$ y/o fueron llevados a cabo antes del auge de las estatinas como fármacos para la reducción de riesgo cardiovascular ${ }^{65}$. El estudio más reciente realizado en una población de pacientes con DM, sin enfermedad cardiovascular establecida, fue el denominado ASCEND, en el cual se aleatorizaron a 15.480 pacientes a recibir AAS $100 \mathrm{mg}$ o placebo. En 7,4 años de seguimiento, el uso de AAS se asoció con un 12\% (RR 0,88 IC 95\%: 0,79-0,97; $p=0,01$ ) de reducción del punto final combinado de IAM, ACVIAIT o muerte de causa vascular con un aumento de sangrados (RR 1,29 IC 95\%: 1,09-1,52; $p=0,003$ ) principalmente gastrointestinales ${ }^{66}$. Un metaanálisis reciente de Abdelaziz sobre 15 estudios aleatorizados (AAS vs control) que incluyó 165.502 pacientes evidenció una reducción de IAM no fatal de 18\%, accidente isquémico transitorio (AIT) $21 \%$ y ACV isquémico $13 \%$, sin impacto en la mortalidad con un aumento en la incidencia de sangrado mayor principalmente gastrointestinal pero sin incremento de sangrados fatales ${ }^{67}$. En este estudio, en el subgrupo de pacientes con DM no se observó beneficio en ninguno de los eventos de eficacia evaluados. Por otro lado, en el último metaanálisis realizado en pacientes con DM (12 ensayos clínicos aleato- rizados, ocho doble ciego y cuatro abiertos) que incluyó 34.227 pacientes con una media de seguimiento de cinco años, en los cuales la dosis de AAS que se utilizó osciló entre 75 y 650 mg diarios, se evidenció en el grupo que recibió AAS una reducción del $11 \%$ en MACE (RR 0,89; IC 95\%: 0,82-0,95) derivando en un número necesario para tratar (NNT) de 95 pacientes, sin evidencia significativa de reducción en el resto de los objetivos incluyendo mortalidad. Cuando se comparó el subgrupo de pacientes que recibió una dosis de AAS $\leq 100 \mathrm{mg}$ se observó una reducción del 25\% en el riesgo de ACV (RR 0,75; 0-59-0,95). Si bien no fue estadísticamente significativo, hubo un aumento del riesgo de sangrados mayores (RR 1,30; IC 95\%: 0,92-1,82) y gastrointestinales (RR 1,48; IC 95\%: 0,87-2,49); se destaca que, pese a no evidenciarse un aumento significativo del riesgo de hemorragia, éste ha sido informado en estudios previos y confirmado por datos de pacientes en mundo real ${ }^{68}$. De lo mencionado anteriormente, se desprende que el uso de AAS en prevención primaria en pacientes con DM no se asoció a reducción en la mortalidad cardiovascular ni total, sino que el beneficio está dado por una menor incidencia de IAM, ACV/AIT, esto a expensas de un aumento del riesgo hemorrágico, que es muy bajo a nivel intracraneal y se da principalmente a expensas de un aumento de sangrados gastrointestinales. En conclusión, el riesgo de sangrado supera al beneficio en la reducción de eventos para indicarlo a todos los pacientes con DM. El impacto de este exceso de sangrado fue abordado por Cea Soriano et al. que evaluaron, en casi 200.000 pacientes (de los cuales 18,1\% tenía DM) que habían comenzado recientemente tratamiento con AAS en bajas dosis, el desarrollo de HDA y baja HDB, y su impacto en mortalidad ${ }^{69}$. Luego de una media de seguimiento de 5,4 años (máximo de 14 años), se identificaron en total 2.721 casos de hemorragia digestiva alta (HDA) y 10.473 de hemorragia digestiva baja (HDB). La incidencia, calculada cada 1.000/personas-año fue de 0,97 HDA y 1,68 HDB, mientras que en el grupo que no recibió AAS fue de 0,67 HDA y 0,76 HDB. Por otro lado, la tasa de mortalidad también expresada en 1.000/personasaño en HDA fue 0,06 y en HDB de 0,01 en el grupo que recibió AAS. En conclusión, el factor más determinante del potencial beneficio clínico del uso de aspirina en prevención primaria en pacientes con DM pareciera ser el riesgo de sangrado. Debi- 
do a la inexactitud intrínseca, a los instrumentos de estimación de riesgo cardiovascular y al aumento del riesgo de sangrado, en prevención primaria, la evidencia que respalda la decisión de utilizar aspirina en bajas dosis en pacientes con DM2 no tiene el mismo tipo de respaldo que en prevención secundaria. Asimismo, debe considerarse que parámetros epidemiológicos como la morbimortalidad de base y la calidad de cuidados que reciben nuestros pacientes difieren de los de los países en los que se origina la evidencia y, por ende, el riesgo cardiovascular de base es mayor. Por lo tanto, la indicación debe ser fruto del análisis del caso individual y con consenso del paciente. Es posible que los sujetos con mayor riesgo de eventos (independientemente del instrumento de estimación de riesgo usado) tengan mayor beneficio clínico neto. Respecto de la dosis, el rango informado en la literatura es de entre 75 y 162 mg diarios. En Argentina, por razones de disponibilidad, la dosis de $100 \mathrm{mg} / \mathrm{día}$ es la recomendada. En los pacientes con alergia documentada a AAS, el uso de clopidogrel $75 \mathrm{mg}$ puede ser una opción. El empleo en prevención secundaria, lo mismo que el de otros antiagregantes (ticagrelor y prasugrel), se discute en la sección de manejo de pacientes con cardiopatía isquémica.

\section{Recomendaciones}

- Está indicado el uso de aspirina en bajas dosis (rango de 75 a 162 mg/día) en pacientes con enfermedad cardiovascular establecida (prevención secundaria) (Grado de recomendación I. Nivel de evidencia A).

- El uso de aspirina en bajas dosis (rango de 75 a $162 \mathrm{mg} / \mathrm{día}$ ) en pacientes con DM sin enfermedad cardiovascular establecida (prevención primaria) podría recomendarse en individuos seleccionados de acuerdo con el riesgo cardiovascular, el riesgo de sangrado y la opinión del paciente (Grado de recomendación Ilb. Nivel de evidencia B).

- En sujetos en prevención primaria con alergia documentada a aspirina puede utilizarse clopidogrel, con las mismas consideraciones hechas en el apartado previo (Grado de recomendación Ilb. Nivel de evidencia C).

\section{d. Enfoque multifactorial}

Los pacientes con DM2 están en alto riesgo de desarrollar complicaciones cardiovasculares, incluida IC. En los últimos años hemos asistido a un cambio en el paradigma de los objetivos del trata- miento de los pacientes con DM2: antes de 2008, el objetivo era la HbA1c "cuanto más baja, mejor"; en 2008 surgió el concepto de evitar hipoglucemia y generar menos ganancia de peso y, finalmente, en 2017 se priorizó la valoración de los factores de riesgo CV como PA y lípidos, aconsejándose lograr un buen control glucémico pero con agentes que brinden seguridad y eficacia cardiovascular.

La enfermedad CV y los factores de riesgo para la enfermedad aterosclerótica son las principales causas de morbilidad y mortalidad en las personas con DM, y representan la mayor contribución a los costos directos e indirectos de la enfermedad. En todos los pacientes con DM, los factores de riesgo cardiovascular deben evaluarse sistemáticamente. Estos factores de riesgo incluyen HTA, dislipidemia, tabaquismo, antecedentes familiares de enfermedad coronaria prematura y albuminuria.

Se observan grandes beneficios cuando se abordan simultáneamente los múltiples factores de riesgo asociados a la DM. El Intensified Multifactorial Intervention in Patients With Type 2 Diabetes and Microalbuminuria (STENO-2) es un estudio clásico de tratamiento multifactorial intensivo en pacientes con DM2. Evaluó 160 pacientes daneses con DM2 y microalbuminuria, tratados de forma intensiva y multifactorial, y seguidos durante 7,8 años. La intervención fue dirigida a controlar factores de riesgo modificables a través de cambios en el estilo de vida e intervención farmacológica individualizada. El resultado obtenido mostró que el control intensivo multifactorial de la glucemia, la PA y los lípidos en pacientes con DM2 aumentó su esperanza de vida, redujo los eventos cardiovasculares y los eventos microvasculares en alrededor del $50 \%$ en el grupo de intervención constatándose una ganancia de vida media de 7,9 años debido fundamentalmente a la reducción o retraso en la aparición de la enfermedad $\mathrm{CV}^{70}$. Más recientemente, en una cohorte nacional sueca, los autores evaluaron la asociación entre el exceso de riesgo de muerte y eventos cardiovasculares entre los pacientes con DM2, de acuerdo con el número de variables de factores de riesgo que lograran encuadrar dentro de los niveles deseables de las pautas terapéuticas, en comparación con los controles emparejados por edad y sexo ${ }^{71}$. En los análisis complementarios, se estimaron la fuerza de las asociaciones entre diversos factores de riesgo y los riesgos incrementales de muerte y los resultados cardiovasculares asociados con la DM. La conclusión del estudio fue que los pacien- 
tes con DM2 que tenían cinco variables de riesgo dentro de parámetros deseables (adecuado control de PA, C-LDL, HbA1C, no fumadores y albuminuria negativa) parecían tener poco o ningún exceso de riesgo de muerte, IAM o $A C V$, en comparación con la población general. Diversos factores pueden contribuir a reducir el riesgo $\mathrm{CV}$ en pacientes con DM2. Las modificaciones en el estilo de vida son la piedra angular en el manejo de la DM2 y para el control de los factores de riesgo cardiovascular. A su vez, los beneficios vasculares derivados de la reducción de lípidos con estatinas y la reducción de la PA son considerablemente mayores que los logrados con la reducción de la glucosa en pacientes con $\mathrm{DM} 2^{72}$. Dada la creciente prevalencia de obesidad, la DM2 se diagnostica con mayor frecuencia en adultos jóvenes y adolescentes. Esta es una tendencia preocupante porque estos individuos tienen peores perfiles de riesgo (IMC, lípidos y niveles de glucemia) al momento del diagnóstico en relación con aquellos pacientes diagnosticados a edades más avanzadas. Por lo tanto, es probable que estos sujetos puedan presentar un riesgo relativamente mayor de mortalidad y morbilidad cardiovascular en comparación con los de mayor edad, tal como demostraron Sattar et al. en un estudio realizado utilizando la Swedish National DM Registry cuyos resultados llevaron a concluir que "el control de los factores de riesgo debe ser más agresivo en las personas que desarrollan DM a edades más tempranas"72.

A manera de conclusión podemos decir, entonces, que la intervención debe efectuarse sobre TODOS los factores de riesgo, no solamente sobre la glucemia, en especial si estamos frente a adultos jóvenes con diagnóstico de DM2 y sosteniendo el tratamiento a lo largo del tiempo.

\section{Recomendaciones}

- En todo paciente con DM debe efectuarse una intervención sobre los diferentes factores de riesgo, no solamente sobre la glucemia, a fin de reducir el riesgo de complicaciones cardiovasculares y microvasculares (Grado de recomendación I. Nivel de evidencia B).

\section{BIBLIOGRAFÍA}

1. Huang $Y$, Cai $X$, Chen $P$, Mai W, Tang H, Huang $Y$, et al. Associations of preDM with all-cause and cardiovascular mortality: $A$ meta-analysis. Annal of med 2014; 46(8):684-692.

2. O'Brien E, Parati G, Stergiou G. European Society of Hypertension Position Paper on ambulatory blood pressure monitoring. Journal of Hypertension 2013; 31:1731-1768.
3. Piepoli M, Hoes W, Agewall S, Albus C, Brotons C, Catapano A, et al. 2016 European Guidelines on Cardiovascular disease prevention in clinical practice. Eur Heart J 2016; 37(29):2315-2381.

4. Cushman WC, Evans GW, Byington RP, Goff DC Jr, Grimm RH Jr, Cutler JA, et al. ACCORD Study Group. Effects of intensive blood-pressure control in type 2 diabetes mellitus. $\mathrm{N}$ Engl J Med 2010; 362(17):1575-1585.

5. Hansson L, Zanchetti A, Carruthers SG, Dahlöf B, Elmfeldt D, Julius $S$, et al. Effects of intensive blood-pressure lowering and low-dose aspirin in patients with hypertension: principal results of the Hypertension OptimalTreatment (HOT) randomised trial. HOT Study Group. Lancet 1998; 351(9118):1755-176.

6. Consejo Argentino Hipertensión. Carlos Braun Menéndez. Consenso de Hipertensión Arterial. Rev Argent Cardiol 2013;81(Supl2):1-72.

7. ESC Guidelines on diabetes mellitus, pre diabetes mellitus and cardiovascular diseases developed in collaboration with the EASD. European Heart Journal 2013; 34:3035-3087.

8. Yusuf S, Teo KK, Pogue J, Dyal L, Copland I, Schumacher H, et al. Telmisartan, ramipril, or both in patients at high risk for vascular events. N Engl J Med 2008; 358(15):1547-1559.

9. Seshasai SR, Kaptoge S, Thompson A, Di Angelantonio E, Gao $\mathrm{P}$, Sarwar N, et al. Diabetes mellitus, fasting glucose, and risk of cause-specific death. N Engl J Med 2011; 364(9):829-4.

10. Haffner SM, Lehto S, RönnemaaT, Pyörälä K, Laakso M. Mortality from coronary heart disease in subjects with type 2 Diabetes Mellitus and in nondiabetic subjects with and without prior myocardial infarction. N Engl J Med 1998; 339(4):229-34.

11. Malmberg K, Yusuf S, Gerstein HC, Brown J, Zhao F, Hunt D, et al. Impact of DM on long-term prognosis in patients with unstable angina and non-Q-wave myocardial infarction: results of the OASIS (Organization to Assess Strategies for Ischemic Syndromes) Registry. Circulation 2000; 102(9):1014-9.

12. Garg A, Grundy SM. Lovastatin for lowering cholesterol levels in non-insulin-dependent diabetes mellitus. N Engl J Med 1988; 318(2):81-86.

13. Knopp RH, Frohlich J, Jokubaitis LA, Dawson K, Broyles FE, Gómez-Coronado D. Efficacy and safety of fluvastatin in patients with non- insulin-dependent diabetes mellitus and hyperlipidemia. Am J Med 1994; 96(6A):69S-78S.

14. Berne C, Siewert-Delle A; URANUS study investigators. Comparison of rosuvastatin and atorvastatin for lipid lowering in patients with type 2 diabetes mellitus: results from the URANUS study. Cardiovasc Diabetol 2005; 4:7-18.

15. Kearney PM, Blackwell L, Collins R, Keech A, Simes J, Peto R, et al. CholesterolTreatmentTrialists' (CTT) Collaborators, Kearney PM, Blackwell L, et al. Efficacy of cholesterol-lowering therapy in 18,686 people with DM in 14 randomised trials of statins: a meta-analysis. Lancet. 2008; 371(9607):117-125.

16. Colhoun HM, Betteridge DJ, Durrington PN, Hitman GA, Neil HA, Livingstone SJ, et al. Primary prevention of cardiovascular disease with atorvastatin in type 2 DM in the Collaborative Atorvastatin DM Study (CARDS): multicentrer randomised placebo-controlled trial. Lancet 2004; 364(9435):685-96.

17. Wanner C, Krane V, Marz W, Olschewski M, Mann JFE, Ruf G, et al. Atorvastatin in patients with type $2 \mathrm{v}$ mellitus undergoing hemodi- alysis. N Engl J Med 2005; 353:238-48.

18. Knopp RH, d'Emden M, Smilde JG, Pocock SJ. Efficacy and safety of atorvastatin in the prevention of cardiovascular end points in subjects with type 2 DM: the Atorvastatin Study for Prevention of Coronary Heart Disease Endpoints in non-insulin-dependent Diabetes Mellitus (ASPEN). Diabetes Care 2006; 29:1478-85.

19. Collins R, Armitage J, Parish S, Sleigh P, Peto R; Heart Protection Study Collaborative Group. MRC/BHF Heart Protection Study of cholesterol-lowering with simvastatin in 5963 people with DM: a randomised placebo-controlled trial. Lancet 2003; 361:2005-16. 
Revista de la Sociedad Argentina de Diabetes Año 55 Vol. 55 № 1 Suplemento Consenso Sociedad Argentina de CardiologíaSociedad Argentina de Diabetes Enero-abril de 2021: 03-70 ISSN 0325-5247 (impresa) ISSN 2346-9420 (en línea)

20. Sever PS, Poulter NR, Dahlöf B, Wedel H, Collins R, Beevers $\mathrm{G}$, et al. Reduction in cardiovascular events with atorvastatin in 2,532 patients with type 2 DM: Anglo-Scandinavian Cardiac Outcomes Trial lipid-lowering arm (ASCOT-LLA). Diabetes Care. 2005; 28:1151-7.

21. Stegman B, Puri R, Cho L, Shao M, Ballantyne CM, Barter PJ, et al. High-intensity statin therapy alters the natural history of diabetic coronary atherosclerosis: insights from SATURN. Diabetes Care 2014; 37:3114-20.

22. Baigent C, Blackwell L, Emberson J, Holland LE, Reith C, Bhala $\mathrm{N}$, et al. Efficacy and safety of more intensive lowering of LDL cholesterol: a meta-analysis of data from 170,000 participants in 26 randomised trials. Lancet 2010; 376:1670-81.

23. Sehayek E. Genetic regulation of cholesterol absorption and plasma plant sterol levels:commonalities and differences. J Lipid Res 2003; 44:2030-2038.

24. Davis HR Jr, Tershakovec AM, Tomassini JE, Musliner T. Intestinal sterol transporters and cholesterol absorption inhibition. Curr Opin Lipidol 2011; 22:467-478.

25. Morrone D, Weintraub WS, Toth PP, Hanson ME, Lowe RS, Lin $\mathrm{J}$, et al. Lipid-altering efficacy of ezetimibe plus statin and statin mono- therapy and identification of factors associated with treatment response: a pooled analysis of over 21,000 subjects from 27 clinical trials. Atherosclerosis 2012; 223:251-61.

26. Hegele RA, Guy J, Ban MR, Wang J. NPC1L1 haplotype is associated with inter-individual variation in plasma low-density lipoprotein response to ezetimibe. Lipids Health Dis 2005; 4:16-21.

27. Gylling $\mathrm{H}$, MiettinenTA. Cholesterol absorption and lipoprotein metabolism in type II diabetes mellitus with and without coronary artery disease. Atherosclerosis 1996; 126:325-32.

28. Lally $\mathrm{S}$, Tan $\mathrm{CY}$, Owens $\mathrm{D}$, Tomkin GH. Messenger RNA levels of genes involved in dysregulation of postprandial lipoproteins in type 2 DM: the role of Niemann-Pick C1-like 1, ATP-binding cassette, transporters G5 and G8, and of microsomal triglyceride transfer protein. Diabetologia 2006; 49:1008-16.

29. Lally SE, Owens D, Tomkin GH. Sitosterol and cholesterol in chylomicrons of type 2 diabetic and non-diabetic subjects: the relationship with ATP binding cassette proteins G5 and G8 and Niemann-Pick C1-like 1 mRNA. Diabetologia 2007; 50:217-9.

30. Tomkin GH. The intestine as a regulator of cholesterol homeostasis in DM. Atherosclerosis 2008; Supplements 9:27-32.

31. Vaverkova $H$, Farnier $M$, Averna $M$, Missault $L$, Viigimaa $M$. Lipid-altering efficacy of ezetimibe/simvastatin $10 / 20 \mathrm{mg}$ compared to rosuvastatin $10 \mathrm{mg}$ in high-risk patients with and without type 2 diabetes mellitus inadequately controlled despite prior statin monotherapy. Cardiovasc Ther 2012; 30:61-74.

32. Fleg JL, Mete M, Howard BV, Umans JG, Roman MJ, Ratner $\mathrm{RE}$, et al. Effect of statins alone versus statins plus ezetimibe on carotid atherosclerosis in type 2 DM: the SANDS (Stop Atherosclerosis in Native Diabetics Study) trial. J Am Coll Cardiol 2008;52: 2198-205.

33. Baigent C, Landray MJ, Reith C, Emberson J, Wheeler DC, Tomson C, et al. The effects of lowering LDL cholesterol with simvastatin plus ezetimibe in patients with chronic kidney disease (Study of Heart and Renal Protection): a randomised placebo-controlled trial. Lancet 2011; 377:2181-92.

34. Giugliano RP, Cannon CP, Blazing MA, Nicolau JC, Corbalán R, Spinar $\mathrm{J}$, et al. Benefit of adding ezetimibe to statin therapy on cardiovas- cular outcomes and safety in patients with versus without Diabetes Mellitus: Results from IMPROVE-IT (Improved Reduction of Outcomes: Vytori Efficacy International Trial). Circulation 2018; 137:1571-1582.

35. Bonaca MP, Gutiérrez JA, Cannon C, Giugliano R, Blazing M, Park JG, et al. Polyvascular disease, type $2 \mathrm{DM}$, and long-term vascular risk: a secondary analysis of the IMPROVE-IT trial. Lancet DM Endocrinol. 2018; 6:934-943.

36. Berglund L, Brunzell JD, Goldberg AC, Murad MH, et al. Evaluation and treatment of hypertriglyceridemia: an endocrine society clinical practice guideline. J Clin Endocrinol Metab 2012; 97:2969-89.
37. Hegele RA, Ginsberg HN, Chapman MJ, Nordestgaard BG, Kuivenhoven JA, Averna M, et al. The polygenic nature of hypertriglyceridaemia: implications for definition, diagnosis, and management. Lancet DM Endocrinol 2014; 2(8):655-66

38. Ginsberg HN, Elam MB, Lovato LC, Crouse 3rd JR, Leiter LA, Linzet $P$, et al. Effects of combination lipid therapy in type 2 diabetes mellitus. N Engl J Med 2010; 362:1563-1574

39. Keech A, Simes RJ, Barter P, Best J, Scott R, Taskinenet MR, et al. Effects of long-term fenofibrate therapy on cardiovascular events in 9,795 people with type 2 diabetes mellitus (the FIELD study): randomised controlled trial. Lancet 2005; 366:1849-1861.

40. Jones $\mathrm{PH}$, Davidson $\mathrm{MH}$. Reporting rate of rhabdomyolysis with fenofibrate + statin versus gemfibrozil + any statin. Am J Cardiol 2005; 95:120-122.

41. Pradhan AD, Paynter NP, Everett BM, Glynn RJ, Amarenco P, Elamet $M$, et al. Rationale and design of the Pemafibrate to Reduce Cardiovascular Outcomes by Reducing Triglycerides in Patients with DM (PROMINENT) study. Am Heart J 2018; 206:80-93.

42. Jun M, Foote C, Lv J, Neal B, Patel A, Nicholls SJ, et al. Effects of fibrates on cardiovascular outcomes: a systematic review and meta- analysis. Lancet 2010; 375:1875-1878.

43. Adkins $Y$, Kelley DS. Mechanisms underlying the cardioprotective effects of omega-3 polyunsaturated fatty acids. J NutrBiochem 2010; 21(9):781-92.

44. Ka H, Song $\mathrm{Y}$, Daviglus ML, Liu K, Van Horn L, Dyeret AR, et al. Accumulated evidence on fish consumption and coronary heart disease mortality: a meta-analysis of cohort studies. Circulation 2004; 109(22):2705-11.

45. Zheng J, Huang T,YuY, Hu X, Yang B, Liet D, et al. Fish consumption and CHD mortality: an updated meta-analysis of seventeen cohort studies. Public Health Nutr 2012;15(4):725-37.

46. Aung T, Halsey J, Kromhout D, Gerstein HC, Marchioli R, Tavazzi $L$, et al. Associations of omega-3 fatty acid supplement use with cardio- vascular disease risks: Meta-analysis of 10 trials involving 77917 individuals. JAMA Cardiol 2018; 3(3):225-234.

47. Abdelhamid AS, Brown TJ, Brainard JS, Biswas P, Thorpe GC, Mooreet $\mathrm{HJ}$, et al. Omega-3 fatty acids for the primary and secondary prevention of cardiovascular disease. Cochrane Database Syst Rev 2018; 11:CD003177.

48. Bowman L, Mafham M, Wallendszus K, Stevens W, Buck G, Barton $\mathrm{J}$, et al. Effects of $\mathrm{n}-3$ fatty acid supplements in diabetes mellitus. N Engl J Med 2018; 379(16):1540-1550.

49. Bhatt DL, Steg PG, Miller M, Brinton EA, Jacobson TA, Ketchumet $\mathrm{SB}$, et al. Cardiovascular risk reduction with icosapent ethyl for hypertriglyceridemia. N Engl J Med 2019; 380(1):11-22.

50. Spolitu S, Dai W, Zadroga JA, Ozcan L. Proprotein convertase subtilisin/kexin type 9 and lipid metabolism. Curr Opin Lipidol 2019; 30:186-191.

51. Steinberg D, Witztum JL. Inhibition of PCSK9: A powerful weapon for achieving ideal LDL cholesterol levels. Proc Natl Acad Sci USA 2009;106:9546-7.

52. Shapiro MD, Tavori H, Fazio S. PCSK9: from basic science discoveries to clinical trials. Circ Res 2018; 122:1420-1438.

53. Navarese EP, Kolodziejczak M, Schulze V, Gurbel PA, Tantry U, LinY, et al. Effects of proprotein convertase subtilisin/kexin type 9 anti-bodies in adults with hypercholesterolemia: A systematic review and meta-analysis. Ann Int Med 2015; 163:40-51.

54. Lipinski MJ, Benedetto U, Escarcega RO, Biondi-Zoccai G, Lhermusier T, Baker NC, et al. The impact of proprotein convertase subtilisin kexin type 9 serine protease inhibitors on lipid levels and outcomes in patients with primary hypercholesterolaemia: A network meta-analysis. Eur Heart J 2016; 37:536-45.

55. Toth PP, Worthy G, Gandra SR, Sattar N, Bray S, Cheng LI. Systematic review and network meta-analysis on the rfficacy of rvolocumab and other therapies for the management of lipid levels in hyperlipidemia. J Am Heart Assoc 2017; 6(10):e005367.

56. Sabatine MS, Giugliano RP, Keech AC, Honarpour N, Wiviott SD, Murphy SA, et al. Evolocumab and clinical outcomes in patients with cardiovascular disease. N Engl J Med 2017; 376:1713-22. 
Revista de la Sociedad Argentina de Diabetes Año 55 Vol. 55 № 1 Suplemento Consenso Sociedad Argentina de CardiologíaSociedad Argentina de Diabetes Enero-abril de 2021: 03-70 ISSN 0325-5247 (impresa) ISSN 2346-9420 (en línea)

57. Schwartz GG, Steg PG, Szarek M, Bhatt DL, Bittner VA, Diaz R, et al. Alirocumab and cardiovascular outcomes after acute coronary syndrome. N Engl J Med 2018 Nov 29; 379(22):2097-2107.

58. Ray KK, Colhoun HM, Szarek M, Baccara-Dinet M, Bhatt DL, Bittner VA, et al. Effects of alirocumab on cardiovascular and metabolic outcomes after acute coronary syndrome in patients with or without DM: a prespecified analysis of the ODYSSEY OUTCOMES randomised controlled trial. Lancet DM Endocrinol 2019;7(8):618-628.

59. Vallejo-Vaz AJ, Ray KK, Ginsberg HN, Davidson MH, Eckel RH, Lee LV, et al. Associations between lower levels of low-density lipopro- tein cholesterol and cardiovascular events in very high-risk patients: Pooled analysis of nine ODYSSEY trials of alirocumab versus control. Atherosclerosis 2019; 288:85-93.

60. Grundy SM, Stone NJ, Bailey AL, Beam C, Birtcher KK, Blumenthal RS. 2018 AHA/ACC. Guideline on the Management of Blood Choles- terol. J Am Coll Cardiol 2019; 73(24): 3168-3209.

61. Piepoli MF, Hoes AW, Agewall S, Albus C, Brotons C, Catapano $A L$, et al. European Guidelines on cardiovascular disease prevention in clinical practice:The Sixth Joint Task Force of the European Society of Cardiology and Other Societies on Cardiovascular Disease Prevention in Clinical Practice (constituted by representatives of 10 societies and by invited experts). European Heart Journal 2016; 37(29):2315-2381.

62. Ogawa H, Nakayama M, Morimoto T, Uemura S, Kanauchi M, Doi N, et al. Low-dose aspirin for primary prevention of atherosclerotic events in patients with type $2 \mathrm{DM}$ : a randomized controlled trial. JAMA 2008; 300:2134-2141.

63. Belch J, MacCuish A, Campbell I, Cobbe S, Taylor R, Prescott R, et al. The prevention of progression of arterial disease and DM (POPA- DAD) trial: factorial randomised placebo controlled trial of aspirin and antioxidants in patients with DM and asymptomatic peripheral arterial disease. BMJ 2008;337:a1840-50.

64. Baigent C, Blackwell L, Collins R, Emberson J, Godwin J, Peto $\mathrm{R}$, et al. Aspirin in the primary and secondary prevention of vascular disease: collaborative meta-analysis of individual participant data from randomised trials. Lancet 2009; 373(9678):1849-60

65. Bowman L, Mafham M, Wallendszus K, Stevens W, Buck G, Barton $\mathrm{J}$, et al. Effects of aspirin for primary prevention in persons with diabetes mellitus. N Engl J Med 2018; 379(16):1529-1539.

66. Abdelaziz H, Saad M, Pothineni V, Megaly M, Potluri R, Saleh $\mathrm{M}$, et al. Aspirin for primary prevention of cardiovascular events. JACC 2019; 73(23):2915-2929.

67. Seidu S, Kunutsor S, Sesso H, Gaziano J, Buring J, Roncaglioni $\mathrm{MC}$, et al. Aspirin has potential benefits for primary prevention of cardiovas- cular outcomes in DM: update literature-based and individual participant data meta-analysis of randomized controlled trials. Cardiovascular Diabetology 2019; 18:70-85

68. Cea Soriano L, Lanas A, Soriano-Gabarró M, García-Rodríguez LA. Incidence of upper and lower gastrointestinal bleeding in new users of low-dose aspirin. Clin Gastroenterol Hepatol 2019;17(5):887-895.

69. Gæde P, Lund-Andersen H, Parving HH and Pedersen O. Effect of a multifactorial intervention on mortality in type $2 \mathrm{DM}$. $\mathrm{N}$ Engl J Med 2008; 358:580-591.

70. Rawshani A, Rawshani A, Franzén S, Sattar N, Eliasson $\mathrm{B}$,Svensson AM, et al. Risk factors, mortality, and cardiovascular outcomes in patients with type 2 DM. N Engl J Med 2018; 379(7):633-644.

71. Li G, Zhang P, Wang J, An Y, Gong Q, Gregg E, et al. Cardiovascular mortality, all-cause mortality, and DM incidence after lifestyle in- tervention for people with impaired glucose tolerance in the Da Qing DM Prevention Study: a 23-year follow-up study. The Lancet DM \& Endocrinology 2014;2(6):474-480.

72. Sattar N, Rawshani A, Franzén S, Rawshani A, Svensson A, Rosengren $A$, et al. Age at diagnosis of type 2 diabetes mellitus and associations with cardiovascular and mortality risks. Circulation 2019; 139(19):2228-2237.

\section{Cardiopatía isquémica}

\section{a. Epidemiología de la DM en síndromes coronarios agudos}

Entre los factores de riesgo coronarios, la DM es el mayor contribuyente de enfermedad coronaria y también de otras manifestaciones de la enfermedad'. Entre el 20 y el $30 \%$ de los pacientes con síndrome coronario agudo (SCA) tienen $\mathrm{DM}^{2}$ y un porcentaje similar presenta intolerancia a la glucosa, o tienen DM pero no fueron diagnosticados antes de la internación ${ }^{3}$. En general, los pacientes con DM son más añosos, tienen más frecuentemente enfermedad cardiovascular preexistente, HTA, dislipidemia y deterioro de la función renal, y se presentan más habitualmente con síntomas atípicos ${ }^{4}$. La DM es más común en pacientes que presentan síndrome coronario agudo sin elevación del segmento ST (SCASEST) ${ }^{5}$ que en los que presentan síndrome coronario agudo con elevación del ST (SCACEST). La DM es un predictor independiente de mala evolución durante un $\mathrm{SCA}^{6}$. Los pacientes con DM tienen mayor riesgo de evolucionar con IC, ACV, reinfarto y sangrado. Además se asocia a mayor mortalidad a 30 días y al año7,8.

La enfermedad coronaria suele ser más difusa y más frecuentemente se manifiesta como enfermedad de tres vasos, lo que puede contribuir a resultados menos favorables con la revascularización $^{9}$. La presencia de DM es un predictor independiente de infarto y muerte ${ }^{10}$. En el contexto de un SCA, los pacientes con diagnóstico previo de DM, como aquellos a quienes ésta se les diagnosticó durante la internación e incluso los pacientes que se presentan con hiperglucemia sin DM, registran mayor mortalidad a 30 días y mayor tasa de nuevos eventos a tres años que la observada en pacientes sin DM con glucemias normales ${ }^{10,11}$. Por otro lado, en aquellos que serán revascularizados en forma quirúrgica, la hiperglucemia en el contexto de un posoperatorio de cirugía cardiovascular se ha asociado a un incremento del riesgo de mediastinitis, sepsis, tiempo en asistencia respiratoria mecánica, estadía hospitalaria e incluso muerte cardiovascular ${ }^{12,13}$.

\section{Tratamiento del SAC en DM}

El tratamiento puede dividirse en:

a) Tratamiento de la hiperglucemia

Mucho se ha discutido si simplemente se trata de un marcador de un paciente más grave o si la 
hiperglucemia genera daño per se. Los avances en el entendimiento de la fisiopatología de la hiperglucemia en situaciones cardiovasculares agudas, así como también algunos ensayos clínicos aleatorizados y estudios observacionales, comprobaron el impacto negativo de la hiperglucemia y la importancia de su manejo adecuado. Ciertas soluciones con insulina como la solución de GIK (insulina-glucosa-potasio) no han demostrado ser eficaces en reducir complicaciones debido a que dichos algoritmos se basan en administrar altas dosis de insulina y, por lo tanto, de glucosa, agua y potasio (con la consiguiente sobrehidratación) con la idea de mejorar la utilización de glucosa por el miocardiocito ${ }^{14}$. Sin embargo, con dichos algoritmos los pacientes persistieron hiperglucémicos por la sobrecarga de glucosa administrada. En cambio, los protocolos de insulinoterapia que intentaban controlar la glucemia y minimizar el riesgo de hipoglucemia demostraron beneficio tanto en pacientes que cursaban un SCA como el posoperatorio de cirugía cardiovascular. De acuerdo con la evidencia actual, obtener controles glucémicos entre 140-180 mg/dL ha señalado tener un adecuado riesgo-beneficio. Se sugiere utilizar insulina endovenosa en goteo continuo mediante protocolos validados en pacientes críticos y esquemas de insulina basal e insulinas rápidas para aquellos no $\operatorname{críticos}^{15}$ (ver protocolos en Anexo II).

\section{Recomendaciones}

- Suspender medicación antidiabética no insulínica en todo paciente con DM internado (Grado de recomendación I. Nivel de evidencia B).

- Mantener valores de glucemia entre 140-180 mg/dL en todo paciente con DM previa o sin ella internado por patología cardiovascular, en especial si se trata de pacientes que cursan internación por síndrome coronario agudo, o en contexto del perioperatorio de cirugía cardiovascular (Grado de recomendación I. Nivel de evidencia B).

- Objetivos más estrictos entre 110-140 mg/dL pueden ser apropiados para pacientes seleccionados, estables, en áreas no críticas, siempre que se eviten riesgos de hipoglucemia significativa (Grado de recomendación I. Nivel de evidencia C).

- En pacientes en áreas críticas, inestables o sin ingesta alimentaria se sugiere utilizar insulina endovenosa en goteo, utilizando protocolos validados (Grado de recomendación I. Nivel de evidencia A).

- En pacientes estables con ingesta oral se su- giere utilizar simultáneamente insulina basal e insulina preprandial para optimizar el control glucémico (Grado de recomendación I. Nivel de evidencia B).

- Se recomienda la implementación de protocolos para manejo y prevención de la hipoglucemia en todas las instituciones (Grado de recomendación I. Nivel de evidencia B).

- Realizar medición de HbA1c en pacientes con DM o hiperglucemia $>140 \mathrm{mg} / \mathrm{dL}$ a la admisión si no fue realizada en los tres meses previos dado que permite guiar el tratamiento antidiabético al alta o sospechar neodiagnóstico de DM (Grado de recomendación I. Nivel de evidencia C).

\section{b) Estrategia de tratamiento coronario (invasivo vs conservador)}

Los pacientes con SCACEST tienen mayor mortalidad con angioplastia transluminal coronaria (ATC) si presentan DM (comparados con pacientes sin DM), pero en ellos también se observó beneficio con ATC primaria en comparación con el tratamiento fibrinolítico ${ }^{16}$. El número necesario para tratar (NNT) a fin de salvar una vida a 30 días es de 17 (IC 95\%: 11-28) en pacientes con DM y de 48 (IC 95\%: 37-60) en aquellos sin DM. Los pacientes con DM tienen más frecuentemente enfermedad de múltiples vasos y la decisión de la revascularización quirúrgica en los SCASEST debe evaluarse según la persistencia de isquemia, inestabilidad hemodinámica o eléctrica, la complejidad de la anatomía coronaria, la dificultad de la ATC, los lechos distales, el monto isquémico, la función ventricular y las comorbilidades. Deben incluirse también los puntajes (scores) que evalúan la complejidad de la anatomía coronaria (SYNTAX) y los predictores clínicos de riesgo (EuroSCORE II y Society of Thoracic Surgeons -STS- $)^{17}$, y participar con un Heart Team para la decisión de la estrategia de reperfusión ${ }^{18}$. En pacientes con DM que presentan enfermedad coronaria de múltiples vasos y bajo riesgo quirúrgico debería preferirse la cirugía sobre la ATC ${ }^{19}$. En los registros, menos del $30 \%$ de los pacientes es sometido a cirugía en el contexto de un SCA$\mathrm{SEST}^{20}$. En pacientes con isquemia persistente se debe optar por la ATC con stents (preferentemente farmacológicos). Los estudios Drug Eluting Stents Evaluation: a randomized trial (DESERT) y Drug Eluting Stenting for Patients with Diabetes Mellitus Trial (Diabetes Trial) $)^{21,22}$ mostraron que la DM es uno de los predictores de mayor riesgo de trombosis del stent en pacientes sometidos a ATC por SCA. 
El monitoreo de nefropatía por contraste es especialmente importante en pacientes con DM luego de un cateterismo, especialmente en los tratados con metformina.

\section{c) Tratamiento antiplaquetario y antitrombótico}

En pacientes con DM2, la resistencia a la insulina y la hiperglucemia participan en la patogénesis del estado protrombótico aumentando el inhibidor del activador del plasminógeno 1 (PAl-1), el factor VII, el factor XII, fibrinógeno, y reduciendo los niveles del activador tisular del plasminógeno (tPA) ${ }^{23}$. Entre los factores que contribuyen a aumentar el riesgo de eventos coronarios en estos pacientes es fundamental la hiperreactividad plaquetaria que se relaciona con up regulation de glicoproteínas (lb y Ilb/llla), P-selectina y los receptores P2Y1224. El tratamiento antiplaquetario dual con aspirina y clopidogrel demostró reducción de infarto, muerte y ACV en el $\mathrm{SCA}^{23}$. Sin embargo, los estudios farmacodinámicos evidenciaron que los pacientes con DM tienen mayor reactividad plaquetaria que aquellos sin $\mathrm{DM}^{25-28}$. Aún duplicando la dosis de clopidogrel en pacientes con DM, la tasa de no respondedores es de un 60\%, lo que obligó a buscar alternativas de antiagregación más potentes ${ }^{29}$. El estudio Optimizing Antiplatelet Therapy in Diabetes Mellitus study (OPTIMUS-3) demostró que el tratamiento con prasugrel $(60 \mathrm{mg}$ como dosis de carga y $10 \mathrm{mg}$ como dosis de mantenimiento) alcanzaba para obtener mayor inhibición plaquetaria que la doble dosis de clopidogrel $600 \mathrm{mg}$ de carga y $150 \mathrm{mg}$ de mantenimiento) ${ }^{30}$. En el subgrupo de 3.416 pacientes con DM del estudio Trial to Assess Improvement in Therapeutic Outcomes by Optimizing Platelet Inhibition With Prasugrel: Thrombolysis in Myocardial Infarction (TRITON-TIMI 38), quienes recibieron prasugrel tuvieron una menor incidencia del punto final combinado de muerte $C V$, IAM o ACV (12,2\% vs 17,0\%; HR: 0,70; $p<0,001)$, diferencia que se observó tanto en pacientes que requerían insulina (14,3\% vs 22,2\%; HR: 0,63; $p=0,009$ ) como en aquellos que no la requerían $(11,5 \%$ vs 15,3\%; HR: 0,74; $p=0,009)$; esto se produjo fundamentalmente a expensas de una reducción en el IAM, sin que hubiera más episodios de sangrado $(2,6 \% \text { vs } 2,5 \% \text {; HR: } 1,06 ; p=0,81)^{34}$. En el estudio PLATelet inhibition and patient Outcomes (PLATO), que comparó pacientes con SCACEST y SCASEST de los cuales el $60 \%$ recibió ATC, en el subgrupo de 4.662 pacientes con DM el evento combinado de muerte CV, IAM o ACV fue menor en el grupo tratado con ticagrelor en comparación con clopidogrel, diferencia que fue significativa sólo en aquellos con HbA1c sobre la media del 6\% (HR: 0,80, 95\% Cl: $0,70-0,91)$ con similar incidencia de sangrado (HR: 0,98, IC 95\%: 0,86-1,12 ${ }^{35}$. En resumen, en los pacientes con DM se describen menores tasas de eventos en quienes reciben prasugrel (en pacientes tratados con ATC) y ticagrelor (en pacientes con y $\sin$ ATC). Los inhibidores de GPIIb/llla demostraron beneficio en pacientes con DM y SCASEST, pero estos estudios se realizaron en la era previa a los antiagregantes actuales, por lo cual debería evaluarse su utilidad en este nuevo contexto.

\section{Recomendaciones}

- En pacientes con DM con SCACEST se debe realizar reperfusión, preferentemente con ATC primaria (Grado de recomendación I. Nivel de evidencia A).

- La estrategia del tratamiento en los pacientes con SCA debe ser independiente del antecedente de DM (Grado de recomendación I. Nivel de evidencia A).

- Se prefiere la estrategia invasiva dentro de las $72 \mathrm{~h}$ en pacientes con SCASEST y DM (Grado de recomendación I. Nivel de evidencia A).

- En la decisión de la estrategia de revascularización de un SCASEST deben tenerse presente las características clínicas y la anatomía. Se recomienda la valoración con puntaje (score) EuroSCORE II y Society of Thoracic Surgeons (STS) y el puntaje SYNTAX en un Heart Team (Grado de recomendación I. Nivel de evidencia A).

- Se sugiere control de la función renal a las $48 \mathrm{~h}$ del procedimiento para diagnóstico de nefropatía por contraste (Grado de recomendación I. Nivel de evidencia A).

- La doble antiagregación plaquetaria debe realizarse con aspirina más prasugrel o ticagrelor en lugar de clopidogrel (Grado de recomendación I. Nivel de evidencia B).

- En pacientes tratados con ATC por IAM se prefiere el tratamiento con ticagrelor o prasugrel sobre el tratamiento con clopidogrel (Grado de recomendación I. Nivel de evidencia A).

- Deben evaluarse el riesgo isquémico y el hemorrágico para la decisión del tratamiento antiplaquetario (Grado de recomendación I. Nivel de evidencia A).

\section{b. Síndromes coronarios crónicos}

Los pacientes con DM y síndromes coronarios crónicos requieren los mismos cuidados que 
aquellos con muy alto riesgo cardiovascular. Esto incluye el control de los factores de riesgo y el control metabólico. La pesquisa de isquemia en pacientes con DM ya fue comentada en el capítulo: Estratificación de riesgo.

\section{Tratamiento farmacológico}

Dado que existen pocos estudios que evaluaron el tratamiento farmacológico específicamente en pacientes con DM y enfermedad coronaria, la mayoría de la información proviene de análisis de subgrupos. El tratamiento debe focalizarse en intensificar el control de los factores de riesgo, con objetivos más estrictos. Las recomendaciones específicas para el manejo de éstos se detallan en las secciones correspondientes de este Consenso. Asimismo, el uso de los fármacos antidiabéticos en pacientes con eventos cardiovasculares previos, y que ha demostrado beneficio en términos de reducción de eventos cardiovasculares, se presenta en la sección correspondiente al algoritmo. El tratamiento específico de la enfermedad coronaria/isquemia es similar al de los pacientes sin DM. Sin embargo, considerando que se trata de una población de mayor riesgo de eventos, el beneficio absoluto es mayor y en consecuencia el número necesario para tratar (NNT) menor ${ }^{36}$. El tratamiento con betabloqueantes parece limitado a pacientes que tienen enfermedad coronaria estable y DM pero con infarto o insuficiencia cardíaca previos ${ }^{37-39}$. No hay evidencias de disminución de la mortalidad en pacientes sin eventos previos.

\section{Tratamiento antiagregante}

Si bien actualmente se discute la profilaxis con aspirina, los pacientes con DM constituyen un subgrupo de riesgo que, si tienen asociada una enfermedad vascular, se benefician con este tratamiento antiagregante; por otro lado, en los pacientes con DM que presentan enfermedad coronaria crónica se describe una menor respuesta a la antiagregación con dosificación tradicional con aspiri$n a^{40}$. Esto se refleja en una inadecuada respuesta antiagregante con una única dosis diaria ${ }^{41}$. Se ha sugerido que la utilización de más de una dosis diaria sería una estrategia correcta para disminuir la reactividad plaquetaria, más eficaz que aumentar la dosis en una única toma ${ }^{42}$. Sin embargo, no existe actualmente ninguna recomendación en este sentido. Además de las alteraciones de la coagulación y las plaquetas estudiadas, Bath et al. sugieren que la formulación y su consecuente absorción tienen un papel clave. Por ello se aconseja utilizar aspirina sin cubierta entérica ${ }^{43}$. En pacientes intolerantes a la aspirina, se sugiere el tratamiento con clopidogrel. En quienes han presentado un SCA hasta tres años antes, podría evaluarse el tratamiento con doble antiagregación con aspirina y ticagrelor $60 \mathrm{mg}$ cada $12 \mathrm{~h}$, especialmente si el evento ocurrió un año antes y el paciente fue tratado con doble antiagregación sin complicaciones de sangrado durante los 12 meses anteriores acorde con los resultados del estudio Prevention of Cardiovascular Events in Patients With Prior Heart Attack Using Ticagrelor Compared to Placebo on a Background of Aspirin-Thrombolysis In Myocardial Infarction 54 (PEGASUS). Este estudio mostró mayor beneficio en pacientes con DM (10,1\% en 90 mg c/12 h vs 10,0\% en 60 mg c/12 h vs $11,60 \%$ en placebo) comparados con no pacientes sin DM $(6,77 \%$ en $90 \mathrm{mg} \mathrm{c} / 12 \mathrm{~h}$ vs $6,68 \%$ en $60 \mathrm{mg} \mathrm{c} / 12 \mathrm{~h}$ vs 7,83\% en placebo) $)^{44}$. El estudio Cardiovascular Outcomes for People Using Anticoagulation Strategies (COMPASS) incluyó 10.341 pacientes con DM y se observó una reducción en el objetivo primario compuesto (MACE) (HR: 0,74, IC 95\%: 0,61-0,9; $p=0,002$ ) con el tratamiento con bajas dosis de rivaroxabán $2,5 \mathrm{mg}$ dos veces al día, asociado a aspirina en pacientes con antecedentes de un evento coronario alejado a expensas de un incremento en la incidencia de sangrados mayores (HR: I,69, IC 95\%: 1,33-2,15; p 0,0006) ${ }^{45}$. El estudio The Effect of Ticagrelor on Health Outcomes in Diabetes Mellitus Patients Intervention Study (THEMIS), que evaluó pacientes con DM y enfermedad coronaria estable, reveló una reducción de MACE del 10\% (HR: 0,90; IC 95\%: 0,81-0,99; $p=0,04$ ) en el grupo asignado a ticagrelor asociado a aspirina, respecto del uso de aspirina sola. Al igual que en el estudio COMPASS, se evidenció un aumento en la incidencia de sangrados mayores (HR: 2,32, IC 95\%: 1,82-2,94; $p<0,001)^{46}$.

\section{Recomendaciones}

- El tratamiento farmacológico antiisquémico no difiere del que se indica en pacientes con enfermedad coronaria sin DM (Grado de recomendación I. Nivel de evidencia A).

- El tratamiento con betabloqueantes queda reservado para pacientes que tienen enfermedad coronaria estable y DM con infarto o insuficiencia cardíaca previos (Grado de recomendación Ila. Nivel de evidencia B). 
- Se recomienda tratamiento antiagregante con aspirina en todos los pacientes con enfermedad coronaria y DM con dosis habituales de 100 mg/día (Grado de recomendación I. Nivel de evidencia A).

- Se recomienda la utilización de aspirina sin cubierta entérica (Grado de recomendación Ila. Nivel de evidencia B).

- En pacientes con DM e infarto previo o ATC puede considerarse el uso de doble antiagregación plaquetaria prolongada con ticagrelor $60 \mathrm{mg}$ cada $12 \mathrm{~h}$ o clopidogrel $75 \mathrm{mg}$ en pacientes con alto riesgo isquémico o bajo riesgo de sangrado (Grado de recomendación lla. Nivel de evidencia B).

- En pacientes con DM y enfermedad cardiovascular con alto riesgo isquémico, en especial aquellos con arteriopatía periférica y bajo riesgo de sangrado, puede considerarse el empleo de rivaroxabán en dosis bajas (Grado de recomendación lla. Nivel de evidencia B).

\section{Estrategias de revascularización}

Revascularización vs tratamiento médico

En enfermedad coronaria estable, el estudio Bypass Angioplasty Revascularization Investigation 2 Diabetes (BARI 2D) aleatorizó 2.368 pacientes con DM a tratamiento médico o revasculari- zación por cirugía de revascularización miocárdica (CRM) o ATC. El método de revascularización fue elegido de acuerdo con el criterio del médico responsable. Entre los pacientes tratados con ATC, sólo el $20 \%$ tuvo intervención en múltiples vasos y se utilizaron BMS en el $56 \%$ de los procedimientos. En el grupo tratado con CRM, el 94\% recibió un bypass de mamaria interna con un promedio de 3,0 anastomosis. En el seguimiento promedio de 5,3 años no se observaron diferencias en la sobrevida (88,3\% en el grupo revascularización vs $87,8 \%$ para tratamiento médico; IC 95\%: -2,0 a 3,$1 ; p=0,97)$ ni en eventos cardiovasculares mayores $(77,2 \%$ vs $75,9 \% ; p=0,7)$. Los pacientes tratados con CRM tuvieron igual sobrevida pero menos eventos cardiovasculares mayores que los asignados a tratamiento médico. Un $42 \%$ de los pacientes asignados a tratamiento médico recibió alguna revascularización antes de los cinco años ${ }^{47}$.

\section{Comparación entre las estrategias}

de revascularización

Diversos estudios compararon la cirugía de revascularización con la intervención percutánea en el tratamiento de la cardiopatía isquémica de pacientes con DM (Tabla 1).

\begin{tabular}{|l|c|c|c|c|}
\hline & Publicación/seguimiento & $\mathbf{n}$ & Mortalidad (\%) & HR p \\
\hline BARI (ATC c/balón) & $1997 / 5$ años & 353 & 34,5 vs 19,5 & $1,8(0,003)$ \\
\hline EAST (ATC c/balón) & $2000 / 8$ años & 59 & 39,9 vs 24,5 & $1,6(0,23)$ \\
\hline CABRI (ATC c/balón) & $2001 / 4$ años & 125 & 22,6 vs 12,5 & $1,8(0,15)$ \\
\hline RITA-1 (ATC c/balón) & $1998 / 7$ años & 62 & 6,9 vs 24,2 & $0,3(0,09)$ \\
\hline ARTS (BMS) & $2005 / 5$ años & 208 & 13,4 vs 8,0 & $1,6(0,27)$ \\
\hline SoS (BMS) & $2008 / 6$ años & 14 & 17,6 vs 5,4 & $3,5(0,15)$ \\
\hline ARTS II (DES) & $2011 / 5$ años & 367 & 9,0 vs 8,6 & $1,0(0,91)$ \\
\hline CARDIA (DES) & $2010 / 1$ año & 50 & 3,2 vs 3,2 & $1,0(0,97)$ \\
\hline SYNTAX (DES) & $2011 / 3$ años & 452 & 13,6 vs 8,7 & $1,6(0,11$ \\
\hline FREEDOM (DES) & $2012 / 5$ años & 1.900 & 16,3 vs 10,9 & $(0,049)$ \\
\hline
\end{tabular}

HR: hazard ratio; ATC: angioplastia transluminal coronaria; BMS: stent metálico; DES: stent farmacológico.

BARI: Bypass Angioplasty Revascularization Investigation; EAST: Early treatment of Atrial fibrillation for Stroke prevention Trial; CABRI: Coronary Artery Bypass Revascularization Investigation; RITA-1: Randomized Intervention Trial of unstable Angina; ARTS: Arterial Revascularization Trial; SoS: Stenting of Saphenous Vein Grafts; CARDIA: Coronary Artery Revascularization in DM; SYNTAX: Synergy between PCI with Taxus and Cardiac Surgery; FREEDOM: Future Revascularization Evaluation in Patients with Diabetes Mellitus: Optimal Management of Multivessel Disease.

Tabla 1: Ensayos clínicos randomizados que compararon estrategias de revascularización. 
Revista de la Sociedad Argentina de Diabetes Año 55 Vol. 55 № 1 Suplemento Consenso Sociedad Argentina de CardiologíaSociedad Argentina de Diabetes Enero-abril de 2021: 03-70 ISSN 0325-5247 (impresa) ISSN 2346-9420 (en línea)

Los estudios Arterial Revascularization Trial I and II (ARTS I y II) incluyeron 367 pacientes con DM. Se compararon en forma no aleatorizada 159 pacientes con enfermedad de múltiples vasos tratados con stent farmacológico (DES, sus siglas en inglés), la cohorte histórica de 96 pacientes tratados con CRM y 112 pacientes tratados con BMS del ARTS I. No se observaron diferencias significativas de mortalidad entre los tres grupos. El IAM fue mayor en el grupo de BMS (BMS 11,0\%, CRM 5,2\%, DES 4,8\%; $p=0,04$ para DES vs BMS y $p=0,76$ para DES vs CRM) y la necesidad de nueva revascularización fue menor en el grupo de CRM (DES 33,2\% vs CRM 10,7\%; $p<0,001)^{48}$. El estudio Coronary Artery Revascularization in DM (CARDIA) aleatorizó a CRM o ATC a 510 pacientes, con enfermedad de múltiples vasos o de un vaso pero compleja. Un tercio de los pacientes del grupo ATC recibió BMS y el resto DES. A un año de seguimiento el punto final combinado de muerte, IAM y ACV fue 10,5\% en el grupo CRM y 13,0\% en el grupo ATC (HR: 1,25, IC 95\%: 0,75-2.,09; $p=0,39$ ), la mortalidad global fue $3,2 \%$ y las tasas del evento combinado de muerte, IAM, ACV y nueva revascularización fueron 11,3 y 19,3\% (HR: 1,77, IC 95\%: 1,11-2,82; $p=0,02)$, respectivamente. Los resultados a cinco años (no publicados) muestran similar mortalidad pero menos IAM y nueva revascularización a favor de la $\mathrm{CRM}^{49,50}$. En el subgrupo preespecificado de pacientes con DM del estudio SYNergy between percutaneous coronary intervention with TAXus and cardiac surgery (SYNTAX) (que incluyó 452 pacientes), la necesidad de nueva revascularización fue menor para la cirugía (ATC 35,3\% vs CRM 14,6\%; p<0,001 HR: 2,01,IC 95\%: $1,04-3,88$ ) pero la mortalidad global (ATC $19,5 \%$ vs CRM 12,9\%; $p=0,065$ ), el ACV (ATC 3,0\% vs CRM $4,7 \% ; p=0,34$ ) o el IAM (ATC 9,0\% vs CRM 5,4\%; $\mathrm{p}=0,20$ ) y el punto final combinado de muerte, infarto o ACV fueron similares (ATC 23,9\% vs CRM 19,1\%; $p=0,21, H R: 1,27-95 \%, I C: 0,84-0,92)$ a cinco años de seguimiento. La mortalidad cardiovascular fue menor con la cirugía (CRM 6,5\% vs ATC 12,7\%, HR: 1,62 95\%, IC 1,03-2,55; $p=0,035$ ), especialmente en los pacientes que requerían insulina. Se observó un gradiente creciente de eventos en relación con la mayor complejidad anatómica de los pacientes. Divididos en tercilos de acuerdo con el puntaje de SYNTAX, la necesidad de nueva revascularización fue mayor en los tres subgrupos, pero el evento combinado de muerte, IAM o ACV sólo fue mayor en los pacientes de mayor complejidad anatómica (SYNTAX mayor de 33) ${ }^{51}$. A tener en cuenta para el análisis de este estudio está el hecho de que los DES utilizados (Taxus) tuvieron una tasa de trombosis aguda y subaguda elevada, y que la doble antiagregación plaquetaria se realizó con aspirina y clopidogrel, fármaco que ha demostrado elevada resistencia en pacientes con DM. El estudio Future Revascularization Evaluation in Patients with Diabetes Mellitus: Optimal Management of Multivessel Disease (FREEDOM) aleatorizó 1.900 pacientes de los cuales el 83\% tenía enfermedad de tres vasos. En el $51 \%$ de los casos se utilizaron stents con sirolimus y en el $43 \%$ con paclitaxel, ambos DES de primera generación. Al cabo de un seguimiento promedio de 3,8 años, la mortalidad global $(18,7 \%$ vs $26,6 \% ; p=0,005)$ y el IAM (6,0 vs $13,9 \% ; p=0,049)$ fueron menores en el grupo CRM. El ACV $(2,4 \%$ vs $5,2 \% ; p=0,03)$ fue menor con la ATC y la mortalidad cardiovascular fue similar (CRM 6,8\% vs ATC 10,9\%; $p=0,02^{52}$ ). Si bien la tasa de eventos fue mayor en los pacientes con DM que requirieron insulina, la ventaja de la CRM se mantuvo para todos ${ }^{53}$. En la interpretación debe considerarse que la mayoría de los pacientes tenía síndromes coronarios estables, que hubo poca inclusión de mujeres, que el grupo quirúrgico tuvo más insuficiencia renal aguda y que nuevamente la doble antiagregación se realizó con clopidogrel. Una revisión de 11 estudios aleatorizados que incluyeron 11.518 pacientes mostró una diferencia en la mortalidad de todas las causas a cinco años en pacientes con DM y enfermedad de múltiples vasos sometidos a CRM vs ATC (10 vs 15,5\%, HR: 1,48; 1,19-1,84; $p=0,0004)^{54}$. El resultado favorable de la CRM con respecto a la ATC puede encontrar explicación en la mayor complejidad de las lesiones en pacientes con DM que predisponen a mayor tasa de reestenosis y necesidad de reintervenciones. Sin embargo, también debe considerarse que hubo una alta tasa de revascularización con puentes mamarios a la arteria descendente anterior (entre 81 y 100\%), lo que mejora la permeabilidad en el mediano y largo plazo, y que se trató de pacientes con riesgo operatorio bajointermedio (EUROSCORE promedio de 3,5) ${ }^{55}$. Los pacientes con DM, con lesiones menos complejas y lesión de uno o dos vasos, pueden ser adecuados candidatos a ATC. 


\section{Resultados con stents de nueva generación}

La utilización de stents de nueva generación ha mejorado los resultados de la ATC, especialmente en los casos de mayor complejidad donde hay evidencia que son capaces de disminuir la mortalidad aún cuando son comparados con los stents de primera generación ${ }^{56}$. En pacientes con DM y enfermedad de múltiples vasos (EMV), los stents de nueva generación muestran progresivamente una reducción de las diferencias con respecto de la CRM en eventos duros, aunque se asocian a mayor tasa de nuevos procedimientos de revascularización ${ }^{57}$. En este sentido, una evidencia más contemporánea surge de un análisis basado en datos individuales de pacientes con DM y enfermedad de múltiples vasos tratados con CRM o ATC utilizando stents con everolimus, en el cual se compararon 8.096 pacientes con similar probabilidad de eventos. En el corto plazo, la ATC tuvo menor mortalidad (HR: 0,58; IC 95\%: 0,34$0,98 ; p=0,04)$ y ACV (HR: 0,14; IC 95\%: 0,06-0,30; $\mathrm{p}<0,0001)$ pero más IAM (HR: 2,44; IC 95\% :1,13$5,31 ; p=0,02)$. En el largo plazo la ATC mostró similar riesgo de muerte $(10,50 \%$ vs $10,23 \%$ HR: 1,12 ; IC 95\%: 0,96-1,30; $p=0,16)$, menos ACV $(2,92 \%$ vs 3,88\% HR: 0,76; IC 95\%: 0,58-0,99; $p=0,04)$ y mayor riesgo de IAM $(6,42 \%$ vs $4,10 \%$ HR: 1,64 ; IC 95\%: 1,32-2,04; $\mathrm{p}<0,0001$ ) y nueva revascularización (21,96\% vs 10,40\% HR: 2,42; IC 95\%: 2,12-2,76 $\mathrm{p}<0,0001)$. La diferencia fue más evidente en los pacientes con revascularización incompleta por ATC ${ }^{58}$.

\section{Estudios que comparan tratamiento médico CRM y ATC}

Un metaanálisis de 5.034 pacientes con enfermedad coronaria estable y DM2, en su mayoría con lesión de múltiples vasos $(77 \%)$, demostró diferencias estadísticamente significativas en el punto final combinado de muerte, infarto o ACV a 4,5 años (CRM + tratamiento médico vs ATC + tratamiento médico HR: 0,71; IC 95\%: 0,590,85; $p=0,0002$ y CRM + tratamiento médico vs tratamiento médico HR: 0,79; IC 95\%: 0,64-0,97; $p=0,022)$. No se observaron diferencias entre tratamiento médico y ATC 59 . Un subanálisis del estudio BARI 2 aplicó el puntaje SYNTAX y demostró que aquellos pacientes con DM y enfermedad coronaria estable se beneficiaban con CRM si el puntaje era mayor de 23 (evento: muerte, infarto o ACV a 5 años, (HR: 1,36; IC 95\%:1,07-1,75, $p=0,01)$. Si el puntaje era $\leq 22$, no había diferencias entre tratamiento médico y la revascularización ${ }^{60}$.

\section{Pacientes con lesión de tronco de coronaria izquierda}

Los pacientes con lesión de tronco de coronaria izquierda (TCI) y DM están subrepresentados en los estudios. Extrapolando los resultados de los 705 pacientes del estudio SYNTAX con lesión de $\mathrm{TCl}^{61}$ y los 3.000 pacientes tratados con stents de última generación en los estudios Nordic-Baltic-British Left Main Revascularization Study (NOBLE) ${ }^{62}$ (15\% con DM) y Evaluation of XIENCE versus Coronary Artery Bypass Surgery for Effectiveness of Left Main Revascularization (EXCEL) (30\% con $D M)^{63}$, observamos que, en lesión de TCl, la cirugía permanece como la estrategia de elección. La ATC a TCl podría ser una opción en casos seleccionados con puntaje SINTAX bajo.

\section{Recomendaciones}

- En pacientes estables con enfermedad coronaria de múltiples vasos, la revascularización puede reducir los eventos cardíacos adversos (Grado de recomendación I. Nivel de evidencia B).

- La CRM es la modalidad preferida de revascularización en pacientes con DM y enfermedad de múltiples vasos (Grado de recomendación I. Nivel de evidencia A).

- La ATC puede considerarse en los pacientes de menor complejidad anatómica (SINTAX bajo y con lesión de uno o dos vasos) (Grado de recomendación lla. Nivel de evidencia B).

- Los stents farmacoactivos de nueva generación deben usarse cuando la opción de revascularización es la ATC de pacientes con DM (Grado de recomendación I. Nivel de evidencia B).

\section{BIBLIOGRAFÍA}

1. Norhammar A, Malmberg K, Diderholm E, Lagerqvist B, Lindahl $B$, Rydén $L$, et al. Diabetes mellitus: The major risk factor in unstable coronary artery disease even after consideration of the extent of coronary artery disease and benefits of revascularization. J Am Coll Cardiol 2004; 43:585-91.

2. García-Aurelio MJ, Cohen-Arazi H, Higa C, Gómez-Santa María HR, Mauro VM, Fernández H, et al. Infarto agudo de miocardio con supradesnivel persistente del segmento ST. Registro multicéntrico SCAR (Síndromes Coronarios Agudos en Argentina) de la Sociedad Argentina de Cardiología. Rev Argent Cardiol 2014; 82:259-267.

3. Giraldez RR, Clare RM, Lopes RD, Dalby AJ, Prabhakaran D, Brogan GX Jr, et al. Prevalence and clinical outcomes of undiagnosed diabetes mellitus and prediabetes among patients with high-risk non-ST-segment elevation acute coronary syndrome. Am Heart J 2013; 165:918-925.

4. Roffi M, Patrono C, Collet JP, Mueller C, Valgimigli M, Andreotti F, et al; ESC Scientific Document Group. 2015 ESC Guidelines for the management of acute coronary syndromes in patients presenting without persistent ST-segment elevation: task force for the management of acute coronary syndromes in patients presenting without persistent ST-segment elevation of the European Society of Cardiology (ESC). Eur Heart J 2016; 37(3):267-315. 
Revista de la Sociedad Argentina de Diabetes Año 55 Vol. 55 № 1 Suplemento Consenso Sociedad Argentina de CardiologíaSociedad Argentina de Diabetes Enero-abril de 2021: 03-70 ISSN 0325-5247 (impresa) ISSN 2346-9420 (en línea)

5. Diercks DB, Owen KP, Kontos MC, Blomkalns A, Chen AY, Miller $C$, et al. Gender differences in time to presentation for myocardial infarction before and after a national women's cardiovascular awareness campaign: a temporal analysis from the Can Rapid Risk Stratification of Unstable Angina Patients Suppress ADverse Outcomes with Early Implementation (CRUSADE) and the National Cardiovascular Data Registry Acute Coronary Treatment and Intervention Outcomes Network- Get with the Guidelines (NCDR ACTION Registry-GWTG). Am Heart J 2010; 160:80-7.

6. Brener SJ, Mehran R, Dressler O, Cristea E, Stone GW, et al. Diabetes mellitus, myocardial reperfusion and outcome in patients with acute ST elevation myocardial infarction treated with primary angioplasty; insights from HORIZON AMI. Am J Cardiol 2012; 109:1111-6.

7. Subherwal S, Bach RG, Chen AY, Gage BF, Rao SV, Newby $\mathrm{LK}$, et al. Baseline risk of major bleeding in non-ST-segmentelevation myocardial infarction: the CRUSADE (Can Rapid risk stratification of Unstable angina patients Suppress ADverse outcomes with Early implementation of the ACC/AHA guidelines) bleeding score. Circulation. 2009; 119:1873-1882.

8. Dotevall A, Hasdai D,Wallentin L, Battler A, Rosengren A. Diabetes mellitus: clinical presentation and outcome in men and women with acute coronary syndromes. Data from the Euro Heart Survey ACS. Diabet Med 2005; 22:1542-1550.

9. Woodfield SL, Lundergan CF, Reiner JS, Greenhouse SW, Thompson MA, Rohrbeck SC, et al. Angiographic findings and outcome in diabetic patients treated with thrombolytic therapy for acute myocardial infarction: the GUSTO-I experience. J Am Coll Cardiol 1996; 28:1661-9.

10. Giraldez RR, Clare RM, Lopes RD, Dalby AJ, Prabhakaran D, Brogan GX Jr, Giugliano RP, James SK, Tanguay JF, Pollack CV Jr, Harrington RA, Braunwald E, Newby LK. Prevalence and clinical outcomes of undiagnosed diabetes mellitus and preDM among patients with high-risk non-ST-segment elevation acute coronary syndrome. Am Heart J 2013; 165:918-925.

11. Bartnik M, Malmberg K, Norhammar A, Tenerz A, Ohrvik J, Ryden L. Newly detected abnormal glucose tolerance: an important predictor of long-term outcome after myocardial infarction. Eur Heart J 2004; 25:1990-1997.

12. Kubal C, Srinivasan AK, Grayson AD, Fabri BM, Chalmers JA. Effect of risk-adjusted DM on mortality and morbidity after coronary artery bypass surgery. Ann Thorac Surg. 2005;79 (5) 1570-1576.

13. Estrada CA, Young JA, Nifong LW, Chitwood WR Jr. Outcomes and perioperative hyperglycemia in patients with or without diabetes mellitus undergoing coronary artery bypass grafting. Ann Thorac Surg 2003; 75 (5):1392-1399.

14. Díaz R, Paolasso EA, Piegas LS, Tajer CD, Moreno MG, Corvalán $R$, et al. Metabolic modulation of acute myocardial infarction. The ECLA (Estudios Cardiológicos Latinoamérica) Collaborative Group. Circulation 1998; 98:2227-34.

15. Puchulu P, Traversa M, Capurro L, Faingold C, González-Infantino $C$, Lobo $\mathrm{P}$, et al. Manejo de la hiperglucemia en el paciente internado. Revista de la Sociedad Argentina de Diabetes MeIlitus. Disponible en: http://www.DM.org.ar/images/Opiniones/ Manejo-HG-paciente-internado.pdf.

16. Timmer JR, Ottervanger JP, de Boer MJ, Boersma E, Grines $\mathrm{CL}$, Westerhout CM, Simes RJ, et al. Primary percutaneous coronary intervention compared with fibrinolysis for myocardial infarction in diabetes mellitus: results from the Primary Coronary Angioplasty vs Thrombolysis-2 trial. Arch Intern Med 2007; 167:1353-1359.

17. 2019 ESC Guidelines on diabetes, pre-diabetes, and cardiovascular diseases developed in collaboration with the EASD. Eur Heart J 2020 Jan 7;v41(2):255-323.

18. Roffi M, Angiolillo DJ, Kappetein AP. Current concepts on coronary revascularization in diabetic patients. Eur Heart J. 2011; 32:2748-2757.

19. Windecker S, Kolh P, Alfonso F, Collet JP, Cremer J, Falk V, et al. 2014 ESC/EACTS guidelines on myocardial revascularization. Eur Heart J 2014; 35:2541-2619.
20. Mancini GBJ, Farkouh ME, Brooks MM, Chaitman BR, Boden WE, Vlachos $\mathrm{H}$, et al. Medical treatment and revascularization options in patients with type $2 \mathrm{DM}$ and coronary disease. Am Coll Cardiol 2016; 68:985-95

21. De Luca G, Dirksen MT, Spaulding C, Kelbæk H, Schalij M, Thuesen L, et al. Impact of DM on long-term outcome after primary angioplasty: insights from the DESERT cooperation. Diabetes Care 2013; 36:1020-5.

22. Jiménez-Quevedo, Sabate M, Angiolillo DJ, et al. Long-term clinical benefit of sirolimus-eluting stent implantation in DM patients with de novo coronary stenosis: long-term results of the DM trial. EHJ 2007; 28:1946-52.

23. Grant PJ. Diabetes mellitus as a prothrombotic condition. J Intern Med 2007; 262:157-172

24. Rivas-Ríos JR, Franchi F, Rollini F, Angiolillo DJ. DM and antiplatelet therapy: from bench to bedside. Cardiovasc DiagnTher 2018; 8(5):594-609.

25. Angiolillo DJ, Jakubowski JA, Ferreiro JL, Tello-Montoliu A, Rollini F, Franchi F, et al. Impaired responsiveness to the platelet P2Y12 receptor antagonist clopidogrel in patients with type $2 \mathrm{DM}$ and coronary artery disease. J Am Coll Cardiol 2014; 64:1005-14.

26. Angiolillo DJ, Fernández-Ortiz A, Bernardo E, Ramírez C, Sabaté $M$, Jiménez-Quevedo $P$, et al. Platelet function profiles in patients with type $2 \mathrm{DM}$ and coronary artery disease on combined aspirin and clopidogrel treatment. Diabetes 2005; 54:2430-5.

27. Angiolillo DJ, Bernardo E, Ramírez C, Costa MA, Sabaté M, Jiménez-Quevedo $P$, et al. Insulin therapy is associated with platelet dysfunc- tion in patients with type 2 diabetes mellitus on dual oral antiplatelet treatment. J Am Coll Cardiol 2006; 48:298-304.

28. Angiolillo DJ, Bernardo E, Sabaté M, Jiménez-Quevedo P, Costa MA, Palazuelos J, et al. Impact of platelet reactivity on cardiovascular outcomes in patients with type 2 diabetes mellitus and coronary artery disease. J Am Coll Cardiol 2007; 50:1541-7.

29. Angiolillo DJ, Shoemaker SB, Desai B, Yuan H, Charlton RK, Bernardo $E$, et al. Randomized comparison of a high clopidogrel maintenance dose in patients with diabetes mellitus and coronary artery disease: results of the Optimizing Antiplatelet Therapy in diabtes Mellitus (OPTIMUS) study. Circulation. 2007;115(6):708-16.

30. Angiolillo DJ, Badimon JJ, Saucedo JF, Frelinger AL, Michelson $A D$, Jakubowski JA, et al. A pharmacodynamic comparison of prasugrel vs high-dose clopidogrel in patients with type 2 diabetes mellitus and coronary artery disease: Results of the Optimizing anti- Platelet Therapy In Diabetes Mellitus (OPTIMUS)-3 Trial. Eur Heart J 2011; 32:838-46.

31. Wiviott SD, Braunwald E, Angiolillo DJ, Meisel S, Dalby AJ, Verheugt FW, et al. Greater clinical benefit of more intensive oral antiplatelet therapy with prasugrel in patients with diabetes meIlitus in the trial to assess improvement in therapeutic outcomes by optimizing platelet inhibition with prasugrel-thrombolysis in myocardial infarction 38. Circulation 2008; 118:1626-36.

32. James S, Angiolillo DJ, Cornel JH, Erlinge D, Husted S, Kontny $\mathrm{F}$, et al. Ticagrelor vs clopidogrel in patients with acute coronary syndromes and DM: A substudy from the PLATelet inhibition and patient Outcomes (PLATO) trial. Eur Heart J 2010, 31:3006-16.

33. Cannon CP, Weintraub WS, Demopoulos LA, Vicari R, Frey MJ, Lakkis N, et al. TACTICS (Treat Angina with Aggrastat and Determine Cost of Therapy with an Invasive or Conservative Strategy)-Thrombolysis in Myocardial Infarction 18 Investigators. Comparison of early invasive and conservative strategies in patients with unstable coronary syndromes treated with the glycoprotein Ilb/IIla inhibitor tirofiban N Engl J Med 2001; 344:1879-87

34. Wiviott SD, Braunwald E, McCabe CH, Montalescot G, Ruzyllo W, Gottlieb S, et al. Prasugrel vs clopidogrel in patients with acute coronary syndromes. New Engl J Med 2007; 357:2001-2015.

35. Wallentin L, Becker RC, Budaj A, Cannon CP, Emanuelsson H, Held C, et al. for the PLATO Investigators. Ticagrelor vs clopidogrel in patients with acute coronary syndromes. N Engl J Med 2009; 361:1045-57. 
Revista de la Sociedad Argentina de Diabetes Año 55 Vol. 55 № 1 Suplemento Consenso Sociedad Argentina de CardiologíaSociedad Argentina de Diabetes Enero-abril de 2021: 03-70 ISSN 0325-5247 (impresa) ISSN 2346-9420 (en línea)

36. Anselmino M, Malmberg K, Ohrvik J, Ryden L. Evidence-based medication and revascularization: powerful tools in the management of patients with DM and coronary artery disease: a report from the Euro Heart Survey on DM and the heart. Eur J Cardiovasc Prev Rehabil 2008; 15:216-223.

37. Shekelle PG, Rich MW, Morton SC, Atkinson CS, Tu W, Maglione $M$, et al. Efficacy of angiotensin converting enzyme inhibitors and beta-blockers in the management of left ventricular systolic dysfunction according to race, gender, and diabetic status: a meta-analysis of major clinical trials. J Am Coll Cardiol 2003; 41:1529-1538.

38. Chen J, Marciniak TA, Radford MJ, Wang Y, Krumholz HM. Betablocker therapy for secondary prevention of myocardial infarction in elderly diabetic patients. Results from the National Cooperative Cardiovascular Project. J Am Coll Cardiol. 1999;34:1388-1394

39. Tsujimoto T, Sugiyama T, Kajio H. Effects of ff-blockers on all cause mortality in patients with type 2 diabetes and coronary heart disease. Diabetes Obes Metab. 2017;19(6):800-808.

40. DiChiara J, Bliden KP,Tantry US, Hamed MS, Antonino MJ, Suarez TA, et al. The effect of aspirin dosing on platelet function in diabetic and nondiabetic patients: an analysis from the aspirin-induced platelet effect (ASPECT) study. Diabetes. 2007; 56:3014-3019]

41. Vernstrøm L, Funck KL, Grove EL, Laugesen E, Baier JM, Hvas $A M$, et al. Antiplatelet effect of aspirin during $24 \mathrm{~h}$ in patients with type $2 \mathrm{DM}$ without cardiovascular disease. Thromb Res 2018 Jan;161:1-6.

42. Bethel MA, Harrison P, Sourij H, Sun Y, Tucker L Kennedy I, et al. Randomized controlled trial comparing impact on platelet reactivity of twice-daily with once-dailyaspirin in people with type 2 diabetes. Diabet Med 2016; 33(2):224-30.

43. Bhatt DL, GrosserT, Dong JF, Logan D, Jeske W, Angiolillo DJ, et al. Enteric coating and aspirin nonresponsiveness in patients with type 2 diabetes mellitus. J Am Coll Cardiol 2017; 69:603-612.

44. Bhatt DL, Bonaca MP, Bansilal S, Angiolillo DJ, Cohen M, Storey $\mathrm{RF}$, et al. Reduction in ischemic events with ticagrelor in diabetic patients with prior myocardial infarction in PEGASUSTIMI 54. J Am Coll Cardiol 2016; 67:2732-2740.

45. Eikelboom JW, Connolly SJ, Bosch J, Dagenais GR, Hart RG, Shestakovska O, et al. Rivaroxaban with or without aspirin in stable cardiovascular disease. N Engl J Med 2017; 377:1319-1330.

46. Steg PG, Bhatt DL, SimonT, Fox K, Mehta SR, Harrington RA, et al. Ticagrelor in patients with stable coronary disease and DM (THEMIS). NEJM 2019; 381:1309-1320.

47. Frye RL, August $P$, Brooks MM, Hardison RM, Kelsey SF, MacGregor JM, et al; BARI 2D Study Group. A randomized trial of therapies for type $2 \mathrm{DM}$ and coronary artery disease. N Engl J Med 2009; 360:2503-15

48. Onuma Y, Wykrzykowska JJ, Garg S, Vranckx P, Serruys PW; ARTS I and II Investigators. 5-year follow-up of coronary revascularization in diabetic patients with multivessel coronary artery disease. JACC Cardiovasc Interv 2011; 4(3):317-323

49. Kapur A, Hall RJ, Malik IS, Qureshi AC, Butts J, de Belder M, et al. Randomized comparison of percutaneous coronary intervention with coronary artery bypass grafting in diabetic patients. 1-year results of the CARDia (Coronary Artery Revascularization in Diabetes) trial. J Am Coll Cardiol 2010; 55(5):432-40.

50. Ellis SG. Coronary revascularization for patients with diabetes: updated data favor coronary artery bypass grafting. J Am Coll Cardiol 2013; 61(8):817-819.

51. Kappetein AP, Head SJ, Morice MC, Banning AP, Serruys PW, Mohr FW, et al. Treatment of complex coronary artery disease in patients with diabetes: 5-year results comparing outcomes of bypass surgery and percutaneous coronary intervention in the SYNTAX trial. Eur J Cardiothorac Surg 2013; 43(5):1006-13.

52. Farkouh ME, Domanski M, Sleeper LA, Siami FS, Dangas G, Mack M, et al. Strategies for multivessel revascularization in patients with DM. N Engl J Med. 2012; 367:2375-84.
53. Dangas GD, Farkouh ME, Sleeper LA, Yang M, Schoos MM, Macaya $\mathrm{C}$, et al. Long Term outcome of $\mathrm{PCl}$ versus CRM in Insulin and Non Insulin treated diabetic patients. J Am Coll Cardiol 2014; 64(12):1189-1197

54. Head SJ, Milojevic M, Daemen J, Ahn JM, Boersma E, Christiansen $\mathrm{EH}$, et al. Mortality after coronary artery bypass grafting ver- sus percutaneous coronary intervention with stenting for coronary artery disease: a pooled analysis of individual patient data. Lancet. 2018;391(10124):939-948. Erratum in: Lancet. 2018 Aug 11; 392(10146):476.

55. Kassimis G, Bourantas CV, Tushar R, de Maria GL, Davlouros $P$, Hahalis G, et al. Percutaneous coronary intervention vs. cardiac surgery in diabetic patients. Where are we now and where should we be going Hellenic J Cardiol 2017; 58:178-189.

56. Piccolo R, Pilgrim T, Heg D, Franzone A, Rat-Wirtzler J, Räber L, et al. Comparative effectiveness and safety of new-generation vs early-generation drug-eluting stents according to complexity of coronary artery disease: a patient-level pooled analysis of 6,081 patients. JACC Cardiovasc Interv 2015; 8(13):1657-66.

57. Bangalore S, Toklu B, Feit F. Outcomes with coronary artery bypass graft surgery versus percutaneous coronary intervention for patients with diabetes mellitus: can newer generation drug-eluting stents bridge the gap? Circ Cardiovasc Interv 2014; 7(4):518-25.

58. Bangalore S, Guo Y, Samadashvili Z, Blecker S, Xu J, Hannan EL. Everolimus eluting stents vs coronary artery bypass gra$\mathrm{ft}$ surgery for patients with diabetes mellitus and multivessel disease. Circ Cardiovasc Interv 2015 Jul; 8(7):e002626. DOI: 10.1161/CIRCINTERVEN-TIONS.115.002626.

59. Mancini GB, Farkouh ME, Brooks MM, Chaitman BR, Boden WE, Vlachos $\mathrm{H}$, et al. Medical treatment and revascularization options in patients with type $2 \mathrm{DM}$ and coronary disease. Am Coll Cardiol 2016; 68:985-95.

60. Ikeno F, Brooks MM, Nakagawa K, Kim MK, Kaneda H, MitsutakeY, et al. SYNTAX score and long-term outcomes: The BARI2DTrial. J Am Coll Cardiol 2017 Jan 31; 69(4):395-403.

61. Mohr FW, Morice MC, Kappetein AP, FeldmanTE, Ståhle E, Colombo A, et al. Coronary artery bypass graft surgery versus percutaneous coronary intervention in patients with three-vessel disease and left main coronary disease: 5 -year follow-up of the randomised, clinical SYNTAX trial. Lancet 2013; 381(9867):629-38.

62. Makikallio T, Holm NR, Lindsay M, Spence MS, Erglis A, Menown IB, et al. Percutaneous coronary angioplasty versus coronary artery bypass grafting in treatment of unprotected left main stenosis (NOBLE): a prospective, randomised, openlabel, non-inferiority trial. Lancet 2016; 388(10061):2743-2752.

63. Stone GW, Sabik JF, Serruys PW, Simonton CA, Genereux P, Puskas J, et al. Everolimus-eluting stents or bypass surgery for left main coronary artery disease. N Engl J Med 2016; 375(23):2223-2235.

\section{Insuficiencia cardíaca y DM}

Entre los pacientes con insuficiencia cardíaca la prevalencia de DM es mucho mayor que en la población general. En diferentes ensayos aleatorizados oscila entre 11 y $40 \%$, y en registros poblacionales entre 25 y $45 \%^{1-3}$. La incidencia de insuficiencia cardíaca es mayor en los pacientes con DM. En el estudio UKPDS, una hemoglobina glicosilada menor del $6 \%$ se asoció a una incidencia de insuficiencia cardíaca de $2 \%$ por año, y una mayor de $10 \%$ a $12 \%$ o por año ${ }^{4}$. La incidencia de IC aumenta de 8 a $16 \%$ por cada $1 \%$ de aumento 
en la hemoglobina glicosilada 5 . En el registro de Framingham, las personas con DM tuvieron mayor riesgo de desarrollar $\mathrm{IC}$ que aquellos sin $\mathrm{DM}^{6}$, con riesgo relativo de 2 para los hombres y 5 para las mujeres (en los menores de 65 años, riesgo relativo de 4 para los hombres y 8 para las mujeres). La relación que existe entre IC y DM va más allá de la estadística7. Los pacientes con IC, cuando tienen DM, presentan mayor compromiso funcional. En ellos, las pruebas de esfuerzo ofrecen peores resultados $^{8}$. La tasa de internación por IC es mayor que en los no diabéticos y la mortalidad también lo es. Esto fue confirmado en diferentes estudios poblacionales, entre ellos los de Rotterdam, Framingham y Olmsted County ${ }^{9-11}$. Un reciente estudio de cohorte con casi 2 millones de pacientes y un seguimiento mediano de 5,5 años reveló que la IC es la segunda manifestación más común en pacientes con DM luego de la enfermedad vascular periférica ${ }^{12}$. En un metaanálisis de siete estudios clínicos y 10 estudios observacionales que incluyó 39.505 pacientes con IC, de los cuales aproximadamente el $24 \%$ tenía DM, el riesgo relativo para mortalidad fue 1,28 y el riesgo relativo para hospitalización $1,36^{13}$. El peor pronóstico de los pacientes con DM se puede atribuir a diferentes causas: mayor prevalencia de factores de riesgo ${ }^{14}$, mayor extensión y severidad de enfermedad coronaria ${ }^{15}$, presencia de una miocardiopatía específica ${ }^{16,17}$ con la coexistencia de dos fenotipos diferentes (cardiopatía diabética con fracción de eyección deprimida o conservada) que responden a diferentes mecanismos fisiopatológicos ${ }^{18}$, y al hecho de que los pacientes con DM suelen estar subtratados, lo cual contribuye a la mayor mortalidad ${ }^{19,20}$.

\section{a. Tratamiento de la insuficiencia cardíaca en los pacientes con DM}

Los diuréticos tiazídicos pueden generar hiperglucemia ${ }^{21}$, pero ello no proscribe su uso ${ }^{22}$. Los antagonistas de la aldosterona no presentan diferencias en la reducción de mortalidad entre pacientes con DM y $\sin \mathrm{DM}^{23}$. El riesgo de generar hiperpotasemia es mayor en los pacientes con DM cuando empleamos antagonistas de la aldosterona, si bien ello no se extiende a formas graves (potasemia $>6 \mathrm{mEq} / \mathrm{L}$ ) o a la necesidad de suspender la medicación si el control del ionograma es frecuente ${ }^{24}$. Los IECA y los antagonistas de la angiotensina II generan igual reducción de mortalidad en pacientes con DM o sin ella cuando se emplean en IC ${ }^{25,26}$. Tanto los IECA como los antagonistas disminuyen la incidencia de DM en pacientes con IC o hipertensos ${ }^{27-31}$. Este efecto puede deberse a la acción directa sobre el páncreas o a un efecto periférico con vasodilatación e incremento, por lo tanto, de la captación de glucosa en la periferiay disminución de los niveles de angiotensina II. Comparado con enalapril, el empleo de sacubitrilo valsartán en el estudio Prospective Comparison of ARNI (Angiotensin Receptor-Neprilysin Inhibitor) with ACEl (Angiotensin-Converting-Enzyme Inhibitor) to Determine Impact on Global Mortality and Morbidity in Heart Failure Trial (PARADIGM) se asoció a igual reducción del punto final primario muerte cardiovascular u hospitalización por IC en pacientes con DM o sin ella, si bien hubo una tendencia a mayor reducción de la mortalidad total en los no diabéticos ${ }^{32}$. En un análisis post hoc del referido estudio, el empleo de sacubitrilo valsartán mostró un mejor control glucémico y menor necesidad de nuevo uso de hipoglucemiantes orales o insulina respecto del empleo de enalapril ${ }^{33}$. En relación a los betabloqueantes en el contexto de la IC, aunque significativa en ambos casos, la disminución de mortalidad es mayor en los pacientes no diabéticos que en aquellos con DM: $28 \%$ frente a $16 \%$. Esto podría contribuir a explicar el peor pronóstico que tienen los pacientes con DM a pesar del tratamiento betabloqueante ${ }^{34}$. El carvedilol, en el estudio Carvedilol or Metoprolol European Trial (COMET), al compararse con metoprolol, demostró superioridad en cuanto a la reducción de la mortalidad en pacientes con DM y sin ella, y disminuir un 22\% la incidencia de nueva $\mathrm{DM}^{35}$. Un metaanálisis de los estudios de carvedilol vs placebo en el contexto de IC sugiere igual efecto de reducción de mortalidad en diabéticos y no diabéticos ${ }^{36}$. La hipoglucemia en los pacientes tratados con betabloqueantes es un efecto adverso poco común, más frecuente en los DM1 y fundamentalmente cuando son lábiles. Por eso, el temor a ella no debe ser un obstáculo para su utilización.

\section{b. Tratamiento de la DM en los pacientes con insuficiencia cardíaca}

En general, la información que se dispone para considerar el efecto de los hipoglucemiantes en pacientes con insuficiencia cardíaca proviene de estudios poblacionales y es retrospectiva. Sólo en los últimos años se han llevado a cabo estudios 
aleatorizados de fármacos hipoglucemiantes en pacientes con insuficiencia cardíaca y DM. El tratamiento con insulina se asocia, en estudios observacionales, a un $25 \%$ de exceso de riesgo de mortalidad respecto del tratamiento con otros fármacos ${ }^{37}$. La razón puede ser que los pacientes tratados con insulina sean pacientes más graves, y por ello debieron ser insulinizados. En algunos estudios aleatorizados de otros fármacos, como Candesartan in Heart Failure Assesment of Reduction in Mortality and Morbidity (CHARM) y Beta-Blocker Evaluation of Survival Trial (BEST), los diabéticos tratados con insulina tuvieron mayor mortalidad ${ }^{38}$. Sin embargo, vale la pena repetir que la administración de insulina no fue aleatoria. En otros estudios retrospectivos no se observó el mismo resultado. En el estudio aleatorizado ORIGIN, el empleo de insulina glargina no apareció asociado a mayor riesgo de insuficiencia cardía$\mathrm{ca}^{39}$. Las sulfonilureas son secretagogos y actúan cerrando canales de potasio ATP-dependientes. Se ha mencionado una serie de riesgos cardiovasculares asociados al uso de sulfonilureas: aumento de tamaño del infarto, disminución de preacondicionamiento y efecto proarrítmico; todos estos efectos adversos se deben fundamentalmente a las sulfonilureas de primera generación. Algunos fármacos más modernos, como por ejemplo la glimepirida, parecen no actuar en los canales cardíacos, razón por la cual el riesgo cardiovascular sería menor ${ }^{40}$. En estudios observaciones, las sulfonilureas, al igual que la insulina, han sido vinculadas a mayor mortalidad y mayor riesgo de generar insuficiencia cardíaca ${ }^{41}$, mientras que en otros no se ha visto esta asociación. Pero vale la misma observación que hicimos antes para la insulina: no se trata de estudios aleatorizados. La metformina ${ }^{42}$ disminuye la gluconeogénesis y la absorción intestinal de glucosa, aumenta la sensibilidad a la insulina y la captación periférica de glucosa. Una reciente revisión sistemática de nueve estudios observacionales con 34.000 pacientes con DM e insuficiencia cardíaca reveló que la metformina se asoció a reducción de mortalidad respecto del tratamiento control (fundamentalmente sulfonilureas): $23 \%$ vs $37 \%$ con OR 0,80 (IC 95\%: 0,74-0,87, $p<0,01)$. No se vio en esta revisión incremento de riesgo en pacientes con peor función ventricular o disfunción renal ${ }^{43}$. El riesgo de acidosis láctica $(9$ por 10.000 personas por año) aumenta en posinfarto agudo de miocardio, insuficiencia cardíaca avanzada o insuficiencia renal. Por eso, a pesar de la precaución que existe en relación a su uso, es una medicación segura, y toda la evidencia observacional sugiere no solamente su seguridad sino también su ventaja respecto de otras intervenciones hipoglucemiantes. La reciente Guía de la Sociedad Europea de Insuficiencia Cardíaca considera el uso de metformina en los pacientes con insuficiencia cardíaca como una indicación lla $C^{44}$. Las tiazolidindionas aumentan la incidencia de insuficiencia cardíaca ${ }^{45}$, tanto la pioglitazona ${ }^{46}$ como la rosiglitazona ${ }^{47}$. Diferentes metaanálisis confirman que existe un riesgo incrementado de desarrollar insuficiencia cardíaca con el uso de tiazolidinedionas. El más completo ${ }^{48}$ considera 29 estudios con 20.254 pacientes y una incidencia de insuficiencia cardíaca de $5,3 \%$ vs 3,7\%, con un OR 1,59 (IC 95\%: 1,34-1,89; $p<0,0001$ ), más alto con rosiglitazona que con pioglitazona (OR de 2,7 vs 1,5, respectivamente). Este incremento del riesgo no se traduce en diferencia en mortalidad. Las recientes guías de práctica clínica proscriben el uso de este grupo de compuestos en todo paciente con insuficiencia cardíaca CF II a IV 44,49. Los inhibidores de la DPP-4 o gliptinas han demostrado una relación variable con la incidencia de insuficiencia cardíaca. En el estudio Saxagliptin Assessment of Vascular Outcomes Recorded in Patients with Diabetes Mellitus Thrombolysis in Myocardial Infarction 53 (SAVOR-TIMI-53) ${ }^{50}$ con saxagliptina hubo exceso de riesgo de presentar insuficiencia cardíaca en el seguimiento, sobre todo en pacientes con niveles más altos de NT-proBNP al inicio del estudio. En el estudio EXAMINE con alogliptina también se verificó tendencia al aumento del riesgo de insuficiencia cardíaca ${ }^{51}$. En cambio, en el estudioTECOS 52 , el uso de sitagliptina no se tradujo en exceso de riesgo. Un metaanálisis de 54 estu$\operatorname{dios}^{53}$ con 74.737 participantes señala tendencia a exceso de riesgo de insuficiencia cardíaca, sin alcanzar el criterio estricto de significación estadística (RR 1,10, IC 95\%: 0,99-1,23; p=0,06). Analizados los fármacos en forma individual, la saxagliptina se asocia a aumento significativo de riesgo ( $R R$ 1,21, IC 95\%: 1,03-1,44) y las otras gliptinas no. Un análisis retrospectivo de casi 1.500.000 pacientes con DM de diversas cohortes de Canadá, Estados Unidos y el Reino Unido54 pone en entredicho el mayor riesgo atribuido a las gliptinas, al señalar que su uso, comparado con el del otros hipoglucemiantes, no incrementó el ries- 
go de hospitalización por insuficiencia cardíaca entre los pacientes con antecedentes de dicha patología o sin ellos. Otro grupo de fármacos es el de los inhibidores del cotransportador 2 sodio-glucosa (SGLT-2), presente en túbulo contorneado proximal ${ }^{55,56}$. Básicamente inhiben la reabsorción renal de sodio y glucosa, por lo que bajan los valores de HbA1c, generan balance calórico negativo, reducción de peso y tensión arterial, disminuyen la albuminuria, los niveles de ácido úrico, la rigidez y resistencia vascular, y ejercen protección de la función renal. Otro mecanismo que se postula es la inhibición del intercambiador sodio-hidrógeno 1, con lo que disminuye la entrada de sodio al interior de la fibra miocárdica y por lo tanto su disponibilidad para ser intercambiado con calcio, con lo que baja la entrada de calcio y se genera un efecto cardioprotector ${ }^{57}$. Tres grandes estudios se llevaron a cabo con gliflozinas en pacientes DM2 con enfermedad aterosclerótica establecida o factores de riesgo para su desarrollo: el estudio EMPA-REG con empagliflozina ${ }^{58}$, el estudio CANVAS con canagliflozina ${ }^{59}$ y el estudio DECLARE con dapagliflozina ${ }^{60}$. Un metaanálisis reciente ${ }^{61}$ considera en total 34.322 pacientes (7.020 de EMPA-REG, 10.142 de CANVAS y 17.160, de DECLARE). En total, el 60,2\% tenía enfermedad vascular establecida y el $39,8 \%$ sólo factores de riesgo (FR). En forma global, las gliflozinas se asociaron a una reducción de la incidencia de eventos adversos cardiovasculares mayores, un compuesto de muerte de origen cardiovascular, infarto agudo de miocardio no fatal y accidente cerebrovascular no fatal, con un HR 0,89, IC 95\%: 0,83-0,96. Sin embargo, la reducción se dio específicamente entre los pacientes con enfermedad instalada (HR: $0,86)$, y no en aquellos con sólo factores de riesgo (HR:1). Al analizar por separado cada uno de los componentes del punto final combinado, se demostró reducción significativa de la mortalidad cardiovascular del orden del $16 \%$ y del IAM no fatal del orden del $11 \%$, sin efecto sobre la incidencia de ACV no fatal. Hubo mayor coincidencia entre ambas fuentes de pacientes en la reducción de la incidencia del punto final mortalidad cardiovascular u hospitalización por insuficiencia cardíaca (HIC) a expensas fundamentalmente de este último componente. Las gliflozinas se asociaron a un HR de 0,76 (IC 95\%: 0,69-0,84) entre los pacientes con enfermedad vascular y de 0,84 (IC 95\%: 0,69-1,01) en aquellos con sólo factores de riesgo.
También fue común la incidencia de un punto final combinado de eventos renales, definido en forma no uniforme pero consistente, en un aumento marcado de la creatinina, la entrada en diálisis o la muerte de origen renal. En los pacientes con enfermedad vascular, el HR fue de 0,56 (IC 95\%: $0,47-0,67) y$, en aquellos con factores de riesgo, de 0,54 (IC 95\%: 0,42-0,71). En dos grandes registros poblacionales, CVD Real 1 y $2^{62,63}$, se ha evidenciado también la asociación del uso de inhibidores SGLT-2 con la disminución de la hospitalización por insuficiencia cardíaca. El estudio Dapagliflozin in Patients with Heart Failure and Reduced Ejection Fraction (DAPA HF) ${ }^{64}$, recientemente publicado, demostró en 4.744 pacientes con insuficiencia cardíaca y fracción de eyección media de $31 \%$ una reducción significativa de un punto final combinado de mortalidad cardiovascular y empeoramiento de la insuficiencia cardíaca (HR: 0,74, IC 95\%: 0,65-0,85), reducción de la mortalidad cardiovascular y de la mortalidad total (HR: 0,83, IC 95\%: 0,71-0,97). Los hallazgos fueron similares en pacientes con DM (un $42 \%$ de pacientes con DM conocidos y un 3\% adicional que fue diagnosticado durante el estudio) y pacientes sin DM. Se abre -a partir de estos resultados- un nuevo panorama para el tratamiento de la insuficiencia cardíaca. Nuevos estudios en insuficiencia cardíaca con fracción de eyección reducida y preservada en los que se testean diversas gliflozinas contribuirán a definir el papel de las gliflozinas más allá de la DM. Con los análogos de los receptores de GLP-1, por último, no se ha visto efecto específico sobre la incidencia de insuficiencia cardíaca. Un metaanálisis reciente ${ }^{65}$ considera ocho estudios aleatorizados. En cinco de ellos se testearon agonistas GLP-1 (ELIXA con lixisenatida, Exenatide Study of Cardiovascular Event Lowerin -EXSCEL- con exenatida, SUSTAIN 6 con semaglutida, HARMONY con albiglutida y LEADER con liraglutida), y en tres, gliflozinas (EMPA-REG Outcome con empagliflozina, CANVAS con canagliflozina y DECLARE con dapagliflozina). En total se incluyeron 77.242 pacientes, el $55,6 \%$ de ellos en los estudios con agonistas GLP-1. La edad media osciló entre 60 y 65 años, la proporción de mujeres fue entre 28 y $40 \%$. La proporción de pacientes con enfermedad aterosclerótica establecida fue $73,1 \%$, pero varió entre el 41 y $100 \%$ según los criterios de inclusión. La prevalencia de insuficiencia cardíaca fue $16,3 \%$ (entre 10 y $24 \%$ según los estudios). El 
Revista de la Sociedad Argentina de Diabetes Año 55 Vol. 55 № 1 Suplemento Consenso Sociedad Argentina de CardiologíaSociedad Argentina de Diabetes Enero-abril de 2021: 03-70 ISSN 0325-5247 (impresa) ISSN 2346-9420 (en línea)

efecto de ambos tipos de fármacos sobre la incidencia de eventos vasculares mayores fue similar: HR: 0,88, IC 95\%: 0,84-0,94 para los agonistas GLP-1 y HR: 0,89, IC 95\%: 0,83-0,96 para las gliflozinas. El efecto se concentró en los pacientes con enfermedad vascular establecida, en los que la reducción alcanzó el 14\% y fue similar con ambas familias de fármacos, mientras que, en aquellos con sólo factores de riesgo, el efecto fue nulo (hallazgo también común a ambos tipos de agentes). Tanto los agonistas GLP-1 como las gliflozinas redujeron significativamente el riesgo de IAM: un $9 \%$ los primeros, un $11 \%$ las gliflozinas, sin evidencia de heterogeneidad entre ambos fármacos. De igual modo ambos redujeron el riesgo de muerte cardiovascular: un 12\% los agonistas GLP1, un 16\% las gliflozinas. Sólo los agonistas GLP-1 disminuyeron el riesgo de ACV un 14\%, mientras que no hubo reducción significativa con las gliflozinas. En cambio, estas últimas disminuyeron significativamente el riesgo de hospitalización por insuficiencia cardíaca un 31\%.

\section{Recomendaciones}

- El tratamiento de la IC en pacientes con DM debe ser igual al de los pacientes sin DM (Grado de recomendación I. Nivel de evidencia A).

- Los inhibidores SGLT-2 son fármacos de primera elección para tratar pacientes con DM con IC o con enfermedad cardiovascular establecida y riesgo incrementado de presentar IC (Grado de recomendación I. Nivel de evidencia A).

- La metformina puede emplearse en pacientes con DM e IC y función renal sin deterioro severo (Grado de recomendación lla. Nivel de evidencia C).

- No deben emplearse tiazolidindionas en pacientes con DM e IC, o propensos a presentarla (Grado de recomendación III. Nivel de evidencia A).

- No debe emplearse saxagliptina en el tratamiento de pacientes con DM con IC, o propensos a presentarla (Grado de recomendación III. Nivel de evidencia B).

\section{BIBLIOGRAFÍA}

1. MacDonald MR, Petrie MC, Hawkins NM, Petrie JR, Fisher M, McKelvie R, et al. Diabetes, left ventricular systolic dysfunction, and chronic heart failure. Eur Heart J 2008; 29(10):1224-40.

2. Baliga V, Sapsford R. Diabetes mellitus and heart failure. An overview of epidemiology and management. Diabetes Vasc Dis Res 2009; 6(3):164-71.
3. Yancy CW, Lopatin M, Stevenson LW, De MarcoT, Fonarow GC. Clinical presentation, management, and in-hospital outcomes of patients admitted with acute decompensated heart failure with preserved systolic function: A report from the Acute Decompensated Heart Failure National Registry (ADHERE) database. J Am Coll Cardiol 2006; 47(1):76-84.

4. Stratton IA, Adler Al, Neil HAW, Matthews DR, Manley SE, Cull $\mathrm{CA}$, et al. Association of glycaemia with macrovascular and microvascular complications of type 2 diabetes (UKPDS 35): prospective. Br Med J 2000; 321:405-12.

5. Iribarren C, Karter AJ, Go AS, Ferrara A, Liu JY, Sidney S, et al. Glycemic control and heart failure among adult patients with diabetes. Circulation 2001; 103(22):2668-73.

6. Kannel WB, Daniel L. Diabetes and cardiovascular disease. The Framingham Study. JAMA J Am Med Assoc 1979; 241(19):2035-8.

7. Cohen-Solal A, Beauvais F, Logeart D. Heart failure and diabetes mellitus: epidemiology and management of an alarming association. J Card Fail 2008; 14(7):615-25.

8. Tibb AS, Ennezat PV, Chen JA, Haider A, Gundewar S, Cotarlan $\mathrm{V}$, et al. Diabetes lowers aerobic capacity in heart failure. J Am Coll Cardiol 2005;46(5):930-1.

9. Mosterd A, Cost B, Hoes AW, de Bruijne MC, Deckers JW, Hofman $A$, et al. The prognosis of heart failure in the general population The Rotterdam Study. Eur Heart J 2001; 22:1318-27.

10. Ho KKL, Anderson KM, Kannel WB, Grossman W, Levy D. Survival after the onset of congestive heart failure in Framingham Heart Study subjects. Circulation 1993; 88(1):107-15.

11. De Groote P, Lamblin N, Mouquet F, Plichon D, McFadden E, Van Belle $E$, et al. Impact of diabetes mellitus on long-term survival in patients with congestive heart failure. Eur Heart J 2004; 25(8):629-30.

12. Shah AD, Langenberg $C$, Rapsomaniki E, Denaxas S, PujadesRodríguez M, Gale CP, et al. Type 2 diabetes and incidence of cardiovascular diseases: A cohort study in 1.9 million people. Lancet Diabetes Endocrinol 2015; 3(2):105-13.

13. Kamalesh M, Cleophas TJ. Heart failure due to systolic dysfunction and mortality in diabetes: pooled analysis of 39,505 subjects. J Card Fail 2009; 15(4):305-9.

14. Adler Al, Stratton IM, Neil HAW, Yudkin JS, Matthews DR, Cull $\mathrm{CA}$, et al. Association of systolic blood pressure with macrovascular and microvascular complications of type 2 diabetes (UKPDS 36): prospective observational study. Br Med J 2000; 321(7258):405-12.

15. Beckman JA, Paneni F, Cosentino F, Creager MA. Diabetes and vascular disease: pathophysiology, clinical consequences, and medical therapy: Part II. Eur Heart J 2013; 34(31):2444-56.

16. Ofstad AP, Urheim S, Dalen H, Orvik E, Birkeland KI, Gullestad $\mathrm{L}$, et al. Identification of a definite diabetic cardiomyopathy in type 2 diabetes by comprehensive echocardiographic evaluation: A cross-sectional comparison with non-diabetic weightmatched controls. J Diabetes 2015; 7(6):779-90.

17. Yilmaz S, Canpolat U, Aydogdu S, Abboud HE. Diabetic cardiomyopathy; Summary of 41 years. Korean Circ J. 2015; 45(4):266-72.

18. Seferovic PM, Paulus WJ. Clinical diabetic cardiomyopathy: A two-faced disease with restrictive and dilated phenotypes. Eur Heart J 2015; 36(27):1718-27.

19. Low Wang CC, Hess CN, HiattWR, Goldfine AB. Clinical update: Cardiovascular disease in diabetes mellitus. Circulation 2016; 133(24):2459-502.

20. Fonarow GC. Managing the patient with diabetes mellitus and heart failure: Issues and considerations. Am J Med 2004; 116(5 SUPPL. 1):76-88.

21. Ramsay LE, Yeo WW, Jackson PR. Diabetes, impaired glucose tolerance and insulin resistance with diuretics. Eur Heart $\mathrm{J}$ 1992; 13(SUPPL. G):68-71.

22. MacDonald MR, Petrie MC, Fisher M, McMurray JJV. Pharmacologic management of patients with both heart failure and diabetes. Curr Heart Fail Rep 2009;6(2):126-32. 
Revista de la Sociedad Argentina de Diabetes Año 55 Vol. 55 № 1 Suplemento Consenso Sociedad Argentina de CardiologíaSociedad Argentina de Diabetes Enero-abril de 2021: 03-70 ISSN 0325-5247 (impresa) ISSN 2346-9420 (en línea)

23. Pitt B, Remme W, Zannad F, Neaton J, Martínez F, Roniker B, et al. Eplerenone, a selective aldosterone blocker, in patients with left ventricular dysfunction after Myocardial infarction. N Engl J Med 2003; 348(14):1695-702.

24. Eschalier R, McMurray JJV, Swedberg K, Van Veldhuisen DJ, Krum H, Pocock SJ, et al. Safety and efficacy of eplerenone in patients at high risk for hyperkalemia and/or worsening renal function: Analyses of the EMPHASIS-HF study subgroups (eplerenone in mild patients hospitalization and survival study in heart failure). J Am Coll Cardiol 2013; 62(17):1585-93.

25. Shekelle PG, Rich MW, Morton SC, Atkinson SW, Tu W, Maglione $M$, et al. Efficacy of angiotensin-converting enzyme inhibitors and beta- blockers in the management of left ventricular systolic dysfunction according to race, gender, and diabetic status: A meta-analysis of major clinical trials. J Am Coll Cardiol 2003; 41(9):1529-38.

26. MacDonald MR, Petrie MC, Varyani F, Ostergren J, Michelson $\mathrm{EL}$, Young JB, et al. Impact of diabetes on outcomes in patients with low and preserved ejection fraction heart failure: An analysis of the Candesartan in Heart failure: Assessment of Reduction in Mortality and morbidity (CHARM) programme. Eur Heart J 2008; 29(11):1377-85.

27. Vermes E, Ducharme A, Bourassa MG, Lessard M, White M,Tardif JC. Enalapril reduces the incidence of diabetes in patients with chronic heart failure: Insight from the studies of left ventricular dysfunction (SOLVD). Circulation 2003; 107(9):1291-6.

28. Yusuf S, Gerstein H, Hoogwerf B, Pogue J, Bosch J, Wolffenbuttel BHR, et al. Ramipril and the development of diabetes. J Am Med Assoc 2001; 286(15):1882-5.

29. Lindholm LH, Ibsen $H$, Borch-Johnsen $K$, Olsen MH, Wachtell $\mathrm{K}$, Dahlöf $\mathrm{B}$, et al. Risk of new-onset diabetes in the losartan intervention for endpoint reduction in hypertension study. $\mathrm{J}$ Hypertens 2002; 20(9):1879-86.

30. Kjeldsen SE, Julius S, Mancia G, Mcinnes GT, HuaT, Weber MA, et al. Effects of valsartan compared to amlodipine on preventing type 2 diabetes in high-risk hypertensive patients: the VALUE trial. J Hypertens 2006; 1405-12.

31. Yusuf S, Ostergren JB, Gerstein HC, Pfeffer MA, Swedberg K, Granger $\mathrm{CB}$, et al. Effects of candesartan on the development of a new diagnosis of diabetes mellitus in patients with heart failure. Circulation 2005; 112(1):48-53.

32. Kristensen SL, Preiss D, Jhund PS, Squire I, Cardoso JS, Merkely $\mathrm{B}$, et al. Risk related to pre-diabetes mellitus and diabetes mellitus in heart failure with reduced ejection fraction: insights from prospective comparison of ARNI with ACEI to determine impact on global mortality and morbidity in heart failure trial. Circ Hear Fail 2016; 9(1):1-12.

33. Seferovic JP, Claggett B, Seidelmann SB, Seely EW, Packer M, Zile MR, et al. Effect of sacubitril/valsartan versus enalapril on glycaemic control in patients with heart failure and diabetes: a post-hoc analysis from the PARADIGM-HF trial. Lancet Diabetes Endocrinol 2017; 5(5):333-40.

34. Haas SJ, VosT, Gilbert RE, Krum H. Are $\beta$-blockers as efficacious in patients with diabetes mellitus as in patients without diabetes mellitus who have chronic heart failure? A meta-analysis of large-scale clinical trials. Am Heart J 2003; 146(5):848-53.

35. Torp-Pedersen C, Metra M, Charlesworth A, Spark P, Lukas MA, Poole-Wilson PA, et al. Effects of metoprolol and carvedilol on preexisting and new onset diabetes in patients with chronic heart failure: Data from the Carvedilol or Metoprolol European Trial (COMET). Heart 2007; 93(8):968-73.

36. Bell DSH, Lukas MA, Holdbrook FK, Fowler MB. The effect of carvedilol on mortality risk in heart failure patients with diabetes: Results of a meta-analysis. Curr Med Res Opin 2006; 22(2):287-96.

37. Smooke S, Horwich TB, Fonarow GC. Insulin-treated diabetes is associated with a marked increase in mortality in patients with advanced heart failure. Am Heart J 2005; 149(1):168-74.

38. Pocock SJ, Wang D, Pfeffer MA, Yusuf S, McMurray JJV, Swedberg KB, et al. Predictors of mortality and morbidity in patients with chronic heart failure. Eur Heart J 2006; 27(1):65-75.
39. Gerstein HC, Bosch J, Dagenais GR, Díaz R, Jung H, Maggioni $A P$, et al. Basal insulin and cardiovascular and other outcomes in dysglycemia. N Engl J Med 2012; 367(4):319-28.

40. Klepzig $H$, Kober $G$, Matter $C$, Luus $H$, Schneider $H$, Boedeker $\mathrm{KH}$, et al. Sulfonylureas and ischaemic preconditioning. $\mathrm{A}$ double-blind, placebo-controlled evaluation of glimepiride and glibenclamide. Eur Heart J 1999; 20(6):439-46.

41. Varas-Lorenzo C, Margulis AV, Pladevall M, Riera-Guardia N, Calingaert $B$, Hazell $L$, et al. The risk of heart failure associated with the use of noninsulin blood glucose-lowering drugs: Systematic review and meta-analysis of published observational studies. BMC Cardiovasc Disord 2014; 14(1).

42. Eurich DT, Tsuyuki RT, Majumdar SR, McAlister FA, Lewanczuk R, Shibata MC, et al. Metformin treatment in diabetes and heart failure: When academic equipoise meets clinical reality. Trials 2009; 10:1-7.

43. Eurich DT, Weir DL, Majumdar SR, Tsuyuki RT, Johnson JA, Tjosvold $L$, et al. Comparative safety and effectiveness of metformin in patients with Diabetes Mellitus and heart failure systematic review of observational studies involving 34,000 patients. Circ Hear Fail 2013; 6(3):395-402.

44. Ponikowski P, Voors AA, Anker SD, Bueno H, Cleland JGF, Coats AJS, et al. 2016 ESC Guidelines for the diagnosis and treatment of acute and chronic heart failure. Eur Heart J 2016; 37(27):2129-2200m.

45. Chaggar PS, Shaw SM, Williams SG. Thiazolidinediones and heart failure. Diabetes Vasc Dis Res 2009; 6(3):146-52.

46. Dormandy JA, Charbonnel B, Eckland DJA, Erdmann E, Massi-Benedetti M, Moules IK, et al. Secondary prevention of macrovascular events in patients with type 2 diabetes in the PROactive Study (PROspective pioglitAzone Clinical Trial in macroVascular Events): A ran- domised controlled trial. Lancet 2005; 366(9493):1279-89.

47. Gerstein HC, Yusuf S, Holman RR, Bosch J, Anand S, Avezum $A$, et al. Effect of rosiglitazone on the frequency of diabetes in patients with impaired glucose tolerance or impaired fasting glucose: A randomised controlled trial. Lancet 2006; 368(9541):1096-105.

48. Hernández AV, Usmani A, Rajamanickam A, Moheet A. Thiazolidinediones and risk of heart failure in patients with or at high risk of type 2 diabetes mellitus: A meta-analysis and metaregression analysis of placebo-controlled randomized clinical trials. Am J Cardiovasc Drugs 2011; 11(2):115-28.

49. Marino J, Barisani JL, Thierer J, Liniado G, Pereir SM, Francesia $A$, et al. Consenso de insuficiencia cardíaca crónica. Rev Argent Cardiol 2016; 84(3):1-50.

50. Scirica BM, Braunwald E, Raz I, Cavender MA, Morrow DA, Jarolim $\mathrm{P}$, et al. Heart failure, saxagliptin, and diabetes mellitus: Observations from the SAVOR-TIMI 53 randomized trial. Circulation 2014;130(18):1579-88.

51. White WB, Cannon CP, Heller SR, Nissen SE, Bergenstal RM, Bakris $\mathrm{GL}$, et al. Alogliptin after acute coronary syndrome in patients with type 2 diabetes. N Engl J Med 2013; 369(14):1327-35.

52. Green JB, Bethel MA, Armstrong PW, Buse JB, Engel SS, Garg $\mathrm{J}$, et al. Effect of sitagliptin on cardiovascular outcomes in type 2 diabetes. N Engl J Med 2015; 373(3):232-42.

53. Kongwatcharapong J, Dilokthornsakul P, Nathisuwan S, Phrommintikul A, Chaiyakunapruk N. Effect of dipeptidyl peptidase-4 inhibitors on heart failure: A meta-analysis of randomized clinical trials. Int J Cardiol 2016; 211:88-95.

54. Filion KB, Azoulay L, Platt RW, Dahl M, Dormuth CR, Clemens $\mathrm{KK}$, et al. A multicenter observational study of incretin-based drugs and heart failure. N Engl J Med 2016; 374(12):1145-54.

55. Heerspink HJL, Perkins BA, Fitchett DH, Husain M, Cherney DZI. Sodium glucose cotransporter 2 inhibitors in the treatment of diabetes mellitus: cardiovascular and kidney effects, potential mechanisms, and clinical applications. Circulation 2016; 134(10):752-72.

56. Marx N, McGuire DK. Sodium-glucose cotransporter-2 inhibition for the reduction of cardiovascular events in high-risk patients with diabetes mellitus. Eur Heart J 2016; 37(42):3192-3200b. 
Revista de la Sociedad Argentina de Diabetes Año 55 Vol. 55 № 1 Suplemento Consenso Sociedad Argentina de CardiologíaSociedad Argentina de Diabetes Enero-abril de 2021: 03-70 ISSN 0325-5247 (impresa) ISSN 2346-9420 (en línea)

57. Packer M. Reconceptualization of the molecular mechanism by which sodium-glucose cotransporter 2 inhibitors reduce the risk of heart failure events. Circulation 2019; 140(6):443-5.

58. Zinman B, Wanner C, Lachin JM, Fitchett D, Bluhmki E, Hantel $S$, et al. Empagliflozin, cardiovascular outcomes, and mortality in type 2 diabetes. N Engl J Med 2015; 373(22):2117-28.

59. Neal B, Perkovic V, Mahaffey KW, De Zeeuw D, Fulcher G, Erondu N, et al. Canagliflozin and cardiovascular and renal events in type 2 diabetes. N Engl J Med 2017; 377(7):644-57.

60. Wiviott SD, Raz I, Bonaca MP, Mosenzon O, Kato ET, Cahn A, et al. Dapagliflozin and cardiovascular outcomes in type 2 diabetes. N Engl J Med 2019; 380(4):347-57.

61. Zelniker TA, Wiviott SD, Raz I, Im K, Goodrich EL, Bonaca MP, et al. SGLT-2 inhibitors for primary and secondary prevention of cardiovascular and renal outcomes in type 2 diabetes: a systematic review and meta-analysis of cardiovascular outcome trials. Lancet 2018; 393(10166):31-9.

62. Kosiborod M, Cavender, MD MA, Fu AZ, Wilding JP, Khunti K, Holl RW, et al. Lower risk of heart failure and death in patients initiated on sodium-glucose cotransporter-2 inhibitors vs other glucose-lowering drugs The CVD-REAL Study (Comparative Effectiveness of Cardiovascular Outcomes in New Users of Sodium-Glucose Cotranspor). Circulation 2017; 136:249-59.

63. Kosiborod M, Lam CSP, Kohsaka S, Kim DJ, Karasik A, Shaw $J$, et al. Cardiovascular events associated with SGLT-2 inhibitors vs other glucose-lowering drugs. J Am Coll Cardiol 2018; 71(23):2628-39.

64. McMurray JJV, Solomon SD, Inzucchi SE, Køber L, Kosiborod MN, Martinez FA, et al. Dapagliflozin in patients with heart failure and reduced ejection fraction. N Engl J Med. 2019; 381(21):1995-2008.

65. ZelnikerTA, Wiviott SD, Raz I, Im K, Goodrich EL, Furtado RHM, et al. Comparison of the effects of glucagon-like peptide receptor agonists and sodium-glucose co-transporter 2 inhibitors for prevention of major adverse cardiovascular and renal outcomes in type 2 diabetes mellitus: a systematic review and meta-analysis of card. Circulation 2019; 2022-31.

\section{Enfermedad vascular periférica y DM}

La importancia de las alteraciones vasculares del paciente con DM está determinada por su elevada prevalencia, alta carga de morbimortalidad y elevados costos en salud?'

\section{Epidemiología}

Las tasas de prevalencia publicadas de enfermedad arterial de miembros inferiores (EAMI) varían ampliamente según el tiempo de evolución de la enfermedad como del control glucémico y de los factores de riesgo en las poblaciones estudiadas ${ }^{2}$.

\section{Impacto de la EAMI en pacientes con DM}

- La prevalencia de la EAMI en pacientes con DM es dos a cuatro veces más frecuente que en pacientes sin DM.

- Un 20\% de las internaciones en pacientes con DM es secundaria a lesiones del pie, secundarias a alteraciones vasculares, neuropáticas o mixtas.
- 8 a $10 \%$ de las personas con DM presentarán en su vida lesiones del pie; las úlceras son responsables de aproximadamente el $85 \%$ de las amputaciones.

- El 50\% de los amputados fallecerá dentro de los tres años siguientes.

- El $50 \%$ de los pacientes con DM y EAMI presenta enfermedad coronaria.

La EAMI del paciente con DM tiene algunas características particulares: son generalmente pacientes más jóvenes, con mayor IMC y más comorbilidades cardiovasculares. A su vez presentan una rápida progresión y la expresión topográfica predominantemente es distal y bilateral. Además, las paredes arteriales suelen estar más calcificadas, con mayor presencia de oclusiones totales. La neo-angiogénesis, como la capacidad de generar circulaciones colaterales compensatorias, es menor en estos pacientes ${ }^{3}$. La presencia de neuropatía periférica incrementa el riesgo de desarrollar úlceras y amputaciones, por lo cual debe valorarse rutinariamente. Del mismo modo requieren especial atención la corrección de anormalidades ortopédicas y otras alteraciones del pie (onicomicosis) que puedan favorecer el desarrollo de un pie diabético.

\section{Presentación clínica}

La claudicación intermitente es el síntoma clásico de la EAMI; sin embargo, puede no ser la presentación habitual del paciente con DM si tiene asociada polineuropatía sensitiva. Además, el dolor neuropático puede confundirse con la claudicación intermitente. En algunas ocasiones, los pacientes pueden debutar con isquemia crítica sin que la claudicación aparezca como aviso previo. Esta última forma de presentación es la manifestación clínica más grave. Dos cualidades caracterizan al paciente con isquemia crítica de miembros inferiores (ICMI): la presencia de enfermedad multisegmentaria, por un lado, y ser paciente de alto riesgo quirúrgico con baja expectativa de vida, por el otro. El aumento en la prevalencia de DM se ha asociado a un incremento en la incidencia de isquemia crítica. En lo que respecta al riesgo cardiovascular, estos pacientes tienen una posibilidad tres veces mayor de desarrollar un SCA, ACV e incluso muerte CV en comparación con los pacientes con claudicación intermitente. La sobrevida libre de amputación es del 50\% al año, el $25 \%$ sufre una amputación y un $25 \%$ fallecerá de causa $\mathrm{CV}^{4,5}$. 
Revista de la Sociedad Argentina de Diabetes Año 55 Vol. 55 № 1 Suplemento Consenso Sociedad Argentina de CardiologíaSociedad Argentina de Diabetes Enero-abril de 2021: 03-70 ISSN 0325-5247 (impresa) ISSN 2346-9420 (en línea)

\section{Diagnóstico}

Luego del interrogatorio y el examen físico se debe confirmar el diagnóstico e intentar estimar el grado de severidad y distribución de la enfermedad en las arterias de miembros inferiores. Dada la frecuencia de pacientes con DM asintomáticos o con síntomas atípicos, se requiere una evaluación más objetiva con las pruebas diagnósticas.

- Índice tobillo-brazo (/TB): en pacientes con DM, la calcificación segmentaria y la rigidez arterial hacen que pueda haber valores de ITB de $\geq 1,4$. Esto es generado por la falta de compresión de la arteria valorada a nivel del tobillo y convierte a estos pacientes en un grupo de alto riesgo de presentar eventos $\mathrm{CV}^{6}$.

- Pruebas de imágenes: el ultrasonido es el método de primera línea para diagnosticar, localizar y cuantificar la EAMI. Sin embargo, debe tenerse en cuenta que es menos precisa en pacientes con DM ya que tiene menor sensibilidad en el territorio infrapatelar y en las zonas de placa calcificada por la sombra acústica que se genera. La angiorresonancia magnética permite evaluar segmentos largos de la anatomía vascular desde el abdomen hasta el tobillo y el calcio no limita el método. Sin embargo, también disminuye su eficacia en el territorio infrapatelar y no puede utilizarse contraste con gadolinio en pacientes con filtrado glomerular $<30 \mathrm{~mL} / \mathrm{min}$ (constituye una contraindicación absoluta para este método). La angiotomografía computarizada presenta un rendimiento diagnóstico menor en el trayecto infrapatelar. Por otro lado, las lesiones altamente calcificadas son difíciles de valorar en forma correcta. La angiografía digital es, en general, el patrón de referencia; hoy en día conserva su lugar en el diagnóstico de aquellos pacientes en los que se estima que se realizará una intervención terapéutica endovascular.

\section{Recomendaciones SAC para diagnóstico de EAMI ${ }^{7}$ Métodos diagnósticos}

Medición del índice tobillo-brazo

\section{Recomendaciones}

- La medición del ITB está indicada como prueba no invasiva de primera línea para detectar y diagnosticar enfermedad arterial de las extremidades inferiores (Clase I. Nivel de evidencia B).

- Ante la presencia de un ITB >1,4 en personas con diabetes o de edad avanzada, debe utilizarse otro método diagnóstico (Clase I. Nivel de evidencia B).

- La medición del ITB con ejercicio debe considerarse en caso de pacientes con síntomas atípicos sugestivos de EVP de los miembros inferiores con el fin de confirmar el diagnóstico y cuantificar la gravedad (Clase Ila. Nivel de evidencia B).

\section{Otros métodos diagnósticos}

\section{Recomendaciones}

- Se recomienda la utilización de ecografía doppler color como método diagnóstico de primera línea para confirmar y localizar la enfermedad arterial de los miembros inferiores (Clase I. Nivel de evidencia B).

- El uso de ultrasonido y tomografía axial computarizada o resonancia magnética nuclear se recomienda para localizar lesiones arteriales en los miembros inferiores y definir la estrategia de revascularización (Clase I. Nivel de evidencia B).

- La indicación de angiografía queda reservada para aquellos pacientes en los que se planea un procedimiento de revascularización (Clase Ila. Nivel de evidencia C).

\section{Tratamiento de la enfermedad arterial periférica de miembros inferiores}

\section{Estrategias terapéuticas}

El tratamiento de la claudicación intermitente está dirigido a aliviar los síntomas, incrementar la distancia caminada y reducir el riesgo cardiovascular global. Los pacientes con EAMI requieren tratamiento médico sistémico para reducir eventos cardiovasculares y tratamiento local de la patología arterial propiamente dicha. Todos deben recibir estrategias de prevención secundaria estrictas y cumplir con las metas definidas para pacientes con enfermedad vascular establecida.

- Ejercicio supervisado: numerosos trabajos prospectivos y aleatorizados han mostrado el beneficio del entrenamiento en la claudicación intermitente. Un metaanálisis recolectó 21 estudios y 
halló un aumento del $179 \%$ en la distancia recorrida hasta el comienzo del dolor y del $122 \%$ a la máxima claudicación, ambas luego de seis meses de entrenamiento. Los programas de ejercicios supervisados son más eficaces que los no supervisados en relación con la mejoría de la distancia caminada y el tiempo máximo de caminata en los pacientes con claudicación intermitente.

- Farmacoterapia: en general, el efecto farmacológico en la distancia caminada es leve a moderado con intervalos de confianza amplios. Los mecanismos de acción no son del todo claros, pero el cilostazol es el fármaco mejor documentado en Argentina. El cilostazol es un inhibidor específico de la fosfodiesterasa-3, que tiene una amplia gama de acciones potencialmente benéficas, incluida la reducción de la agregación plaquetaria, así como la potenciación de la vasodilatación, aunque el mecanismo exacto es desconocido. Un metaanálisis más reciente incluyó datos combinados originales de nueve ensayos controlados aleatorizados que evaluaron cilostazol para la claudicación intermitente. Un total de 1.258 pacientes fueron tratados con cilostazol $100 \mathrm{mg}$ cada $12 \mathrm{~h}$; en conjunto, mejoró la distancia de caminata máxima en un 50,7\% en comparación con una mejoría de $24,3 \%$ con placebo y un aumento absoluto de 42,1 metros con respecto al placebo $(p<0,001)^{8}$.

La SAC ha publicado recomendaciones específicas sobre el tratamiento médico de la EAMI?. Respecto del uso de antidiabéticos, es menester señalar que uno de los estudios con canagliflozina evidenció un incremento del riesgo de amputaciones menores. Por ello se sugiere la individualización del tratamiento en los pacientes con EAP.

\section{Revascularización en la enfermedad vascular periférica}

En el paciente con claudicación intermitente deberá considerarse la revascularización ante la persistencia de síntomas luego de tres meses bajo tratamiento médico (ejercicio y cilostazol) ${ }^{9,10}$. La presencia de isquemia crítica es indicación de revascularización con el objetivo de aliviar el dolor y evitar la amputación. Los pilares del manejo son la reconstrucción arterial y el salvataje del miembro, lo cual debe efectuarse sin dilación ${ }^{11}$. El pie diabético es una entidad clínica específica que puede involucrar, además de la enfermedad arterial periférica, neuropatía periférica, traumatismo, alteraciones ortopédicas, inflamación, infección o la combina- ción de éstos. El manejo de esta patología requiere cuidados multidisciplinarios, desde el control de los factores de riesgo cardiovascular, la revascularización cuando fuese posible, hasta la optimización del cuidado de las úlceras, el uso apropiado de calzado, el tratamiento de la infección y la rehabilitación. Las recomendaciones específicas sobre la revascularización están detalladas en el documento correspondiente publicado por la SAC?

\section{BIBLIOGRAFÍA}

1. Jude EB, Eleftheriadou I, Tentolouris N. Peripheral arterial disease in diabetes. A review. Diabet Med 2010; 27(1):4-14.

2. Scatena A, Petruzzi P, Ferrari M, Rizzo L, Cicorelli A, Berchiolli $\mathrm{R}$, et al. Outcomes of three years of teamwork on critical limb ischemia in patients with diabetes and foot lesions. Int J Low Extrem Wounds 2012; 11(2):113-9.

3. Aiello A, Anichini R, Brocco E, Caravaggi C, Chiavetta A, Cioni $R$, et al. Treatment of peripheral arterial disease in diabetes: A consensus of the Italian Societies of Diabetes (SID, AMD), Radiology (SIRM) and Vascular Endovascular Surgery (SICVE). Nutr Metab Cardiovasc Dis 2014; 24(4):355-69.

4. Brevetti G, Schiano V, Chiariello M. Endothelial dysfunction: A key to the pathophysiology and natural history of peripheral arterial disease? Atherosclerosis 2008; 197(1):1-11.

5. Lepäntalo M, Fiengo L, Biancari F. Peripheral arterial disease in diabetic patients with renal insufficiency: a review. Diabetes Metab Res Rev 2012; 28(Suppl 1):40-5.

6. Jeppsson A, Sandoval E, Landoni G, Head SJ, Dunning J, Landmesser U, et al. 2017 EACTS Guidelines on perioperative medication in adult cardiac surgery. Eur J CardioThoracic Surg 2017; 53(1):5-33.

7. Cura F, Belcastro F, Bluro I, Ferreira M, Levalle-Cobo AM, Mosso $\mathrm{G}$, et al. Consenso de Enfermedad Vascular Periférica. Rev Argent Cardiol 2015; 83(3).

8. Pande RL, Hiatt WR, Zhang P, Hittel N, Creager MA. A pooled analysis of the durability and predictors of treatment response of cilostazol in patients with intermittent claudication. Vasc Med 2010; 15(3):181-8.

9. Faglia E, Mantero M, Caminiti M, Caravaggi C, De Giglio R, Pritelli $C$, et al. Extensive use of peripheral angioplasty, particularly infrapop- liteal, in the treatment of ischaemic diabetic foot ulcers: Clinical results of a multicentric study of 221 consecutive diabetic subjects. J Intern Med 2002; 252(3):225-32.

10. Faglia E, Dalla Paola L, Clerici G, Clerissi J, Graziani L, Fusaro $M$, et al. Peripheral angioplasty as the first-choice revascularization procedure in diabetic patients with critical limb ischemia: Prospective study of 993 consecutive patients hospitalized and followed between 1999 and 2003. Eur J Vasc Endovasc Surg 2005; 29(6):620-7.

11. Ferraresi R, Centola M, Ferlini M, Da Ros R, Caravaggi C, AssaIoni R, et al. Longterm outcomes after angioplasty of isolated, below the knee arteries in diabetic patients with critical limb ischaemia. Eur J Vasc Endovasc Surg 2009; 37(3):336-42.

\section{Accidente cerebrovascular isquémico y DM}

La DM triplica el riesgo de ACV isquémico a 10 años, incrementando el riesgo tanto para el ACV 
lacunar como el de gran arteria, predominando clásicamente la primera clase ${ }^{1-3}$. Este riesgo es mayor cuando coexiste con HTA (Tabla 1).

\begin{tabular}{|l|l|}
\hline Variable & Cocientes de riesgo $($ IC 95\%) \\
\hline HTA sola (TAS 140-159 mm Hg) & $1,29(1,13-1,46)$ \\
\hline HTA sola (TAS $\geq 160 / 95 \mathrm{~mm} \mathrm{Hg})$ & $1,93(1,48-4,16)$ \\
\hline DM sola & $2,48(1,48-4,16)$ \\
\hline DM e HTA (TAS $140-159 \mathrm{~mm} \mathrm{Hg})$ & $4,26(2,90-6,25)$ \\
\hline DM e HTA (TAS $\geq 160 \mathrm{~mm} \mathrm{Hg})$ & $4,90(3,87-6,21)$ \\
\hline
\end{tabular}

TAS: tensión arterial sistólica; IC: intervalo de confianza; DM: diabetes mellitus.

Tabla 1: Cociente de riesgos (hazard ratio) para la incidencia de ACV isquémico dependiendo de antecedentes de HTA y $D^{4}{ }^{4}$.

Entre los factores vinculados al aumento del riesgo, la DM puede acelerar la ateromatosis por vías inflamatorias a través de la producción de radicales libres y la inhibición de la óxido nítrico sintetasa, exponiendo el endotelio a estrés por cizallamiento ${ }^{5}$. Por otra parte, la hiperglucemia y la DM tienen repercusiones diferentes en el resultado del ACV isquémico y hemorrágico ${ }^{6}$. La hiperglucemia al ingreso se asocia con un peor resultado clínico y un mayor riesgo de muerte hospitalaria en pacientes con ACV tanto isquémico como hemorrágico, mientras que la DM aumenta el riesgo de muerte hospitalaria en pacientes con ACV hemorrágico, pero no en pacientes con ACV isquémicos.

\section{Control glucémico}

El correcto control de la DM es fundamental en la reducción del riesgo de estos pacientes. Así, los pacientes con HBA1c > 7\% presentan mayor mortalidad; es por eso que, mediante modificaciones en el estilo de vida y la farmacoterapia, se busca adecuar el control glucémico con un objetivo para la HBA $1 \mathrm{c} \leq 7 \%$ planteándose objetivos inferiores a $6,5 \%$ en pacientes seleccionados siempre que se minimice el riesgo de hipoglucemia?.

\section{Presión arterial}

Las recomendaciones generales sobre el manejo de la presión arterial están citadas en el capítulo correspondiente. Se deben conseguir cifras de PA $<140 / 90 \mathrm{mmHg}$, aunque en sujetos con DM es probable que se beneficien de un control más estricto. El estudio Action to Control Cardiovascular. Risk in Diabetes-Blood Pressure (ACCORD BP) ${ }^{8}$ de- mostró que un mayor control con PAS $<120 \mathrm{mmHg}$ reduce el riesgo de $\mathrm{ACV}$ isquémico en un $41 \%$ en pacientes con $\mathrm{DM}$ y factores de riesgo $\mathrm{CV}$, a expensas de un aumento del riesgo de insuficiencia renal (número necesario para generar un daño-NNH: 50). Un metaanálisis de 31 ensayos que incluyó 73.913 individuos con $\mathrm{DM}^{9}$ demostró que una reducción más intensa de la PA no redujo la tasa de IAM pero disminuyó significativamente el riesgo de ACV. Otro metaanálisis ${ }^{10}$ determinó que, en pacientes con DM2 y/o alteración de la glucemia en ayunas y/o alteración de la tolerancia a la glucosa, el tratamiento con un objetivo de PAS de 130 a $135 \mathrm{mmHg}$ es aceptable. Sin embargo, con objetivos más estrictos $(<130 \mathrm{mmHg})$ se observó heterogeneidad en la afectación de órgano blanco, ya que el riesgo de ACV siguió disminuyendo, pero no hubo beneficio con respecto del riesgo de otros eventos macrovasculares y microvasculares (cardíacos, renales y retinianos), mientras que el riesgo de eventos adversos graves aumentó. Por lo tanto, se recomienda lograr cifras de PA <140/90 mmHg; un tratamiento más intensivo con objetivos menores debe destinarse únicamente a pacientes seleccionados y con un estricto seguimiento clínico.

\section{Fibrilación auricular}

La DM es uno de los factores que, combinados, aumentan el riesgo de ACV en pacientes con fibrilación auricular (FA), sumando un punto en la puntuación de CHA2DS2-VASc ${ }^{11}$.

\section{Tratamiento antidiabético}

En pacientes con DM y ACV relacionado a aterosclerosis se aconseja, luego de la metformina, indicar agentes que hayan demostrado una reducción significativa en los eventos vasculares ateroscleróticos en los ensayos clínicos. Estos agentes son los iSGLT-2 y arGLP-1 (ver sección de algoritmos de manejo de hiperglucemia en el paciente con DM). Los ensayos clínicos aleatorizados que evaluaron seguridad cardiovascular con estos grupos farmacológicos tuvieron como criterios de inclusión el antecedente de ACV y se observó, en estos pacientes con eventos previos, disminución de eventos cardiovasculares combinados. Por otra parte, algunos arGLP-1 como dulaglutida en el estudio Researching Cardiovascular Events with a Weekly Incretin in Diabetes (REWIND) y semaglutida en el estudio SUSTAIN han demostrado, en análisis secundarios, reducción de ACV. Algunos metaanálisis realizados a partir de 
los ensayos clínicos con arGLP-1 han descrito este impacto sobre $A C V^{12,13}$. Es importante resaltar que no contamos aún con estudios diseñados específicamente para evaluar el efecto de estos agentes en pacientes con ACV previo. La pioglitazona (una tiazolidindiona) también demostró reducción de eventos cardiovasculares tanto en pacientes con DM con eventos previos (estudio PROspective pioglitAzone Clinical Trial In macroVascular Events, PROactive) como en pacientes sin DM con insulinorresistencia y ACV isquémico previo (estudio Insulin Resistance Intervention after Stroke, IRIS) ${ }^{14}$. Sin embargo, algunos eventos adversos relacionados con las glitazonas limitan su uso.

\section{Glucemia y DM en el ACV agudo}

La glucemia elevada al ingreso $(\geq 144 \mathrm{mg} / \mathrm{dL})$ se asoció con resultados clínicos desfavorables a tres meses en pacientes con ACV isquémico, tanto con DM como sin DM, tratados con trombolisis endovenosa $^{15}$. Se recomiendan concentraciones séricas de glucosa en el rango de 140 a $180 \mathrm{mg} / \mathrm{dL}$ durante las primeras $24 \mathrm{~h}$ en todos los pacientes hospitalizados. Por lo general, la hiperglucemia en el contexto del accidente cerebrovascular agudo se trata con insulinoterapia.

\section{BIBLIOGRAFÍA}

1. Banerjee C, Moon YP, Paik MC, Rundek T, Mora-McLaughlin C, Vieira JR, et al. Duration of diabetes and risk of ischemic stroke: the Northern Manhattan Study. Stroke 2012; 43:1212-1217.

2. OhiraT, Shahar E, Chambless LE, Rosamond WD, MosleyTH, Folsom AR. Risk factors for ischemic stroke subtypes: the atherosclerosis risk in communities study. Stroke 2006; 37(10):2493-8

3. Janghorbani M, Hu FB, Willett WC, Li TY, Manson JE, Logroscino $\mathrm{G}$, et al. Prospective study of type 1 and type 2 diabetes and risk of stroke subtypes:The Nurses' Health Study. Diabetes Care 2007; 30(7):1730-1735.

4. Hu G, Sarti C, Jousilahti P, Peltonen M, Qiao Q, Antikainen R, et al. The impact of history of hypertension and type 2 diabetes at baseline on the incidence of stroke and stroke mortality. Stroke 2005; (12):2538-2543.

5. Woo C-H, Shishido T, McClain C, Lim JH, Li J-D, Yang J, et al. Extracellular signal-regulated knase 5 SUMOylation antagonizes shear stress induced antiinflammatory response and endothelial nitric oxide synthase expression in endothelial cells. Circulation Research 2008; 102:538-545

6. Snarska KK, Bachórzewska-Gajewska H, Kapica-Topczewska K, Drozdowski W, Chorffy M, Kułakowska A, et al. Hyperglycemia and diabetes have different impacts on outcome of ischemic and hemorrhagic stroke. Arch Med Sci 2017; 13:100-108.

7. Garber AJ, Abrahamson MJ, Barzilay JI, Blonde L, Bloomgarden ZT, Bush MA, et al. Consensus statement by the american association of clinical endocrinologists and american college of endocrinology on the comprehensive type 2 DM management algorithm - 2018 executive summary. Endocr Pract 2018; 24:91-120.

8. ACCORD Study Group, et al. Effects of intensive blood-pressure control in type 2 diabetes mellitus. New Engl J Med 2010; 362:1575-1585.
9. Reboldi G, Gentile G, Angeli F, Ambrosio G, Mancia G, Verdecchia P. Effects of intensive blood pressure reduction on myocardial infarction and stroke in diabetes: a meta-analysis in 73,913 patients. Journal of Hypertension 2011; 29(7)1253-1269.

10. Bangalore S, Kumar S, Lobach I, Messerli FH. Blood pressure targets in subjects with type 2 diabetes mellitus/impaired fasting glucose: observations from traditional and bayesian random-effect meta-analyses of randomized trials. Circulation 2011; 123:2799-810.

11. Lip GYH, Nieuwlaat R, Pisters R, Lane DA, Crijns HJG. Refining clinical risk stratification for predicting stroke and thromboembolism in atrial fibrillation using a novel risk factor-based approach: the euro heart survey on atrial fibrilation. Chest 2010; 137(2):263-272

12. Barkas $F$, Elisaf M, Milionis H. Protection against stroke with glucagon-like peptide 1 receptor agonists: a systematic review and meta- analysis. Eur J Neurol 2019; 26(4):559-565.

13. Lim S, Oh TJ, Dawson J, Sattar N. Diabetes drugs and stroke risk: Intensive versus conventional glucose-lowering strategies, and implications of recent cardiovascular outcome trials. Diabetes Obes Metab 2020; 22(1):6-15.

14. American Diabetes Association. Cardiovascular disease and risk management: Standards of Medical Care in Diabetes-2018. Diabetes Care 2018; 41:S86-S104.

15. Tsivgoulis G, Safouris A, Kim D-E, Alexandrov AV. Recent advances in primary and secondary prevention of atherosclerotic stroke. J Stroke 2018; 20(2):145-166.

\section{Arritmias y DM}

La DM incrementa el riesgo de enfermedad cardiovascular y también el de sus complicaciones. Los pacientes con DM están expuestos a experimentar eventos arrítmicos ventriculares y supraventriculares, así como también episodios sincopales de causa no arrítmica. En esta sección repasamos los aspectos salientes de estos temas.

\section{a. Fibrilación auricular}

La fibrilación auricular (FA) es la arritmia sostenida más frecuente de la población general y produce una mayor morbilidad y mortalidad ${ }^{1}$. El estudio Framingham demostró que la DM es un predictor independiente para el desarrollo de FA (OR 1,4 hombres y 1,6 mujer $)^{2}$. Huxley et al. también mostraron un incremento del RR de FA de 1,24 (1,06-1,40), con una incidencia de FA/aleteo auricular del $14,9 \%$ vs $2,5 \%$ en el grupo control ${ }^{3-5}$. Asimismo, los pacientes con DM suelen presentar obesidad, lo que contribuye a un mayor riesgo de FA; una adecuada estrategia de control de medidas higiénico/dietéticas permite que disminuya su incidencia ${ }^{6}$. En relación con el mecanismo, no existe una explicación sencilla para dicha relación, por lo cual se sugieren varias hipótesis fisiopatológicas. Primero, la fibrosis auricular desempeña un papel preponderante para el inicio y perpetuación de la FA. En este sentido, el estrés oxidativo 
Revista de la Sociedad Argentina de Diabetes Año 55 Vol. 55 № 1 Suplemento Consenso Sociedad Argentina de CardiologíaSociedad Argentina de Diabetes Enero-abril de 2021: 03-70 ISSN 0325-5247 (impresa) ISSN 2346-9420 (en línea)

en pacientes con DM se encuentra incrementado, y consecuentemente los aniones superóxido, promoviendo la fibrosis auricular. Andersen et al. detectaron un aumento del estrés oxidativo mitocondrial en la aurícula de pacientes con DM, lo que permite sostener esta hipótesis?. Segundo, la glicosilación no enzimática de las proteínas y su unión a receptores de factores de crecimiento de tejido conectivo producen un aumento de la fibrosis auricular ${ }^{8}$. Tercero, la disfunción autonómica producto de la DM es un trastorno conocido que conduce a un aumento del estrés catecolaminérgico cardiovascular ${ }^{9}$. En este sentido, la misma disautonomía se convierte en un factor que predispone al desarrollo de FA. Otro mecanismo relacionado con el desarrollo de FA es la alteración en la conducción intraauricular e interauricular. Chao et al. estudiaron a 228 pacientes con DM que requerían ablación de FA con navegador tridimensional, y observaron que los pacientes presentaban menor voltaje auricular que un grupo control y una mayor tasa de recurrencia arrítmica luego de la ablación ${ }^{10}$. Estos hallazgos demuestran un compromiso intrínseco eléctrico auricular asociado con la DM. Con respecto a los niveles de glucemia, existen controversias acerca de si los pacientes con hiperglucemia sostenida o con picos de glucemia, o bien los que se encuentran bajo tratamiento hipoglucemiante intensivo o no, son más o menos propensos a desarrollar FA. La glucemia pareciera ser un marcador de enfermedad más que un factor para el desarrollo de FA. Un punto importante a considerar es que la relación entre DM y FA se asocia con un aumento de eventos tromboembólicos, principalmente una mayor incidencia de ACV ${ }^{11}$. De hecho, la DM se considera un marcador de riesgo tromboembólico en todos los puntajes de riesgo (CHADS2, CHA2DS2-VASC). Este hecho resulta relevante a los fines de una terapéutica anticoagulante preventiva. La DM es una enfermedad que se asocia a otras enfermedades CV y hace posible una superposición de mecanismos para el desarrollo de FA sin que pueda hallarse una única causa determinante. Con respecto al tratamiento de la FA en pacientes con DM, no existe una terapéutica distinta de la referida en las guías de recomendación de FA de la Sociedad Argentina de Cardiología, teniendo presente que el control estricto de la DM permitiría una menor incidencia de la arritmia ${ }^{12}$.

\section{b. Arritmias ventriculares}

La enfermedad cardiovascular es la principal causa de muerte en pacientes con DM, y la DM es casi un sinónimo de aterosclerosis y enfermedad coronaria. La alta incidencia y extensión de la enfermedad coronaria en estos pacientes implica una elevada incidencia de arritmias ventriculares y muerte súbita cardíaca. En el Paris Prospective Study I, la presencia de DM incrementó 1,82 veces (IC 95\%: 1,04-3,17) el riesgo de muerte súbita, ajustado por otros factores de riesgo conocidos $^{13}$. La muerte por enfermedad coronaria también está incrementada en casi cuatro veces y este riesgo crece ante la mayor duración de la $\mathrm{DM}^{14}$. En un metaanálisis de 14 estudios observacionales que analizó más de 5.000 pacientes, la presencia de DM duplicó el riesgo de muerte súbita ${ }^{15}$. En pacientes con DM con fracción de eyección del ventrículo izquierdo (FEVI) $>35 \%$, la incidencia de muerte súbita es similar a la de pacientes sin DM con $\mathrm{FEVI}<35 \%{ }^{16}$. Si bien esta asociación se basa principalmente en la extensión de la enfermedad coronaria en los pacientes con DM, otros mecanismos pueden intervenir en este incremento del riesgo de arritmias ventriculares y muerte súbita. Algunos estudios informaron prolongación del intervalo QT y alternancia de la onda T en pacientes con DM. Estos trastornos de la repolarización ventricular son conocidos predictores de eventos arrítmicos malignos y muerte súbita, y pueden generar un sustrato para arritmias ventriculares independiente del antecedente de IAM previo ${ }^{17,18}$. La neuropatía diabética es otro factor que predispone a la aparición de arritmia ventricular al generar un desequilibrio autonómico con predominio del tono simpático ${ }^{19}$. Además de favorecer un sustrato arritmogénico, la DM puede también participar como gatillo de episodios arrítmicos. Las oscilaciones en el estado glucémico podrían actuar como disparadores de arritmias ventriculares. Tanto niveles elevados de HbA1c como episodios de hipoglucemia se han asociado con episodios espontáneos de arritmia ventricular $^{20-22}$. En resumen, la DM se asocia a mayor incidencia de arritmia ventricular más allá de la esperada por la mayor incidencia de enfermedad coronaria.

\section{c. Síncope}

El síncope y la DM están íntimamente asociados, dado que las lesiones que provoca la DM en los grandes nervios a lo largo de la enfermedad llevan al síncope. La neuropatía autonómica es una complicación frecuente de la DM. Esta neuropatía es causada por el deterioro de la regulación autonómica de las fibras nerviosas que regulan la frecuencia cardíaca, el gasto cardíaco, la contractilidad miocárdica, y la dilatación y la constricción de los vasos sanguíneos. 
Esto causa un gran rango de trastornos entre los que se incluye el síncope. La neuropatía diabética es una neuropatía de grandes nervios y su primera manifestación es la afección del nervio vago, que es responsable del $75 \%$ de la actividad parasimpática. La consecuencia de la denervación de los nervios eferentes simpáticos es la hipotensión ortostática que es una causante muy común de síncope en diabéticos. El tilt test puede ser útil para diagnosticar esta hipotensión ortostática dado que hay varios patrones distintos recientemente descritos ${ }^{23}$.

El tratamiento de la hipotensión ortostática incluye medidas generales como la ingesta importante de agua, y evitar cambios posturales bruscos, ejercicios que aumenten la presión abdominal y fármacos que puedan provocar hipotensión.

\section{BIBLIOGRAFÍA}

1. Kannel WB, Abbott RD, Savage DD, McNamara PM. Epidemiologic features of chronic atrial fibrillation: the Framingham study. N Engl J Med 1982; 306:1018-1022.

2. Benjamin EJ, Levy D, Vaziri SM, D’Agostino RB, Belanger AJ, Wolf PA. Independent risk factors for atrial fibrillation in a population-based cohort. The Framingham Heart Study JAMA 1994; 271(11):840-4.

3. Huxley RR, Filion KB, Konety S, Alonso A. Meta-analysis of cohort and case-control studies of type 2 diabetes mellitus and risk of atrial fibrillation. Am J Cardiol 2011; 108(1):56-62.

4. Movahed MR, Hashemzadeh M, Jamal MM. Diabetes mellitus is a strong, independent risk for atrial fibrillation and flutter in addition to other cardiovascular disease. Int J Cardiol 2005; 105(3):315-8.

5. Pallisgaard JL, Schjerning AM, LindhardtTB, Procida K, Hansen ML, Torp-Pedersen C, Gislason GH. Risk of atrial fibrillation in diabetes mellitus: A nation wide cohort study. Eur J Prev Cardiol 2016; 23(6):621-7.

6. Pathak RK, Middeldorp ME, Lau DH, Mehta AB, Mahajan R, Twomey $D$, et al. Aggressive risk factor reduction study for atrial fibrillation and implications for the outcome of ablation: the ARREST-AF cohort study. J Am Coll Cardiol 2014; 64:2222-31.

7. Anderson EJ, Kypson AP, Rodríguez E, Anderson CA, Lehr EJ, Neufer PD. Substrate-specific derangements in mitochondrial metabolism and redox balance in the atrium of the type 2 diabetic human heart. J Am Coll Cardiol 2009; 54(20):1891-1898.

8. KatoT,Yamashita T, Sekiguchi A,TsunedaT, Sagara K, Takamura M, et al. AGEs-RAGE system mediates atrial structural remodeling in the diabetic rat. J Cardiovasc Electrophysiol 2008; 19(4):415-420.

9. Pop-Busui R. Cardiac autonomic neuropathy in diabetes: a clinical perspective. Diabetes Care 2010; 33(2):434-441.

10. Chao TF, Suenari K, Chang SL, Lin YJ, Lo LW, Hu YF, et al. Atrial substrate properties and outcome of catheter ablation in patients with paroxysmal atrial fibrillation associated with diabetes mellitus or impaired fasting glucose. Am J Cardiol 2010; 106:1615-1620.

11. Varughese GI, Patel JV, Tomson J, Lip GYH. The prothrombotic risk of diabetes mellitus in atrial fibrillation and heart failure. $J$ Thromb Haemost 2005; 3:2811-3.

12. Hadid C, González J, Abello M, Muratore C, Giniger A, Dubner S, et al. Consenso de fibrilación auricular. Sociedad Argentina de Cardiología. Rev Argent Cardiol 2015; 83(Supl 1).

13. Balkau $B$, Jouven $X$, Ducimetière $P$, Eschwège $E$. Diabetes as a risk factor for sudden death. Lancet 1999; 354:1968-1969.
14. Cho E, Rimm EB, Stampfer MJ, Willett WC, Hu FB. The impact of diabetes mellitus and prior myocardial infarction on mortality from all causes and from coronary heart disease in men. J Am Coll Cardiol 2002; 40(5): 954-960.

15. Zaccardi F, Khan H, Laukkanen JA. Diabetes mellitus and risk of sudden cardiac death: A systematic review and meta-analysis. Int J Cardiol 2014;177(2):535-537.

16. Junttila MJ, Barthel $P$, Myerburg RJ, et al. Sudden cardiac death after myocardial infarction in patients with type 2 diabetes. Heart Rhythm 2010; 7(10):1396-1403.

17. Cardoso CR, Salles GF, Deccache W. Prognostic value of QT interval parameters in type 2 diabetes mellitus: results of a longterm follow-up prospective study. J Diabetes Complications 2003;17(4):169-178.

18. Molon G, Costa A, Bertolini L, Zenari L, Arcaro G, Barbieri E, et al. Relationship between abnormal microvolt T-wave alternans and poor glycemic control in type 2 diabetic patients. Pacing Clin Electrophysiol 2007; 30(10):1267-1272.

19. Vinik Al, Ziegler D. Diabetic cardiovascular autonomic neuropathy. Circulation 2007;115: 387-397.

20. Stahn A, Pistrosch F, Ganz X, Teige M, Koehler C, Bornstein S, et al. Relationship between hypoglycemic episodes and ventricular arrhythmias in patients with type 2 diabetes and cardiovascular diseases: silent hypoglycemias and silent arrhythmias. Diabetes Care 2014; 37(2):516-520.

21. Pistrosch F, Ganz X, Bornstein SR, Birkenfeld AL, Henkel E, Hanefeld M. Risk of and risk factors for hypoglycemia and associated arrhythmias in patients with type $2 \mathrm{DM}$ and cardiovascular disease: a cohort study under real-world conditions. Acta Diabetol 2015; 52: 889-895.

22. Chen-Scarabelli C, Scarabelli TM. Suboptimal glycemic control, independently of QT interval duration, is associated with increased risk of ventricular arrhythmias in a high-risk population. $\mathrm{Pa}$ cing Clin Electrophysiol 2006; 29(1):9-14.

23. Teodorovich $\mathrm{N}$, Swissa M. Tilt table test today-state of the art. World J Cardiol. 2016; 8(3): 277-282.

\section{Complicaciones microvasculares e impacto cardiovascular}

La presencia de complicaciones microvasculares relacionadas con la DM se asocia al desarrollo de complicaciones cardiovasculares, por lo cual debe considerarse como un factor de riesgo cardiovascular mayor. Diversos estudios observacionales demostraron una asociación entre retinopatía proliferativa severa, nefropatía diabética y neuropatía diabética, y un mayor riesgo de mortalidad cardiovascular ${ }^{1}$. El peor control metabólico y/o el mayor tiempo de evolución de DM que estos pacientes presentan, así como también la presencia de otros factores de riesgo en común, explican en parte esta asociación. A su vez complicaciones como la nefropatía diabética y la neuropatía autonómica cardíaca, sobre las cuales nos referiremos con mayor detalle, pueden (por diferentes procesos fisiopatológicos) favorecer el desarrollo en forma directa de diversas complicaciones cardiovasculares. En conclusión, es importante efectuar un adecuado screening de complicaciones 
microvasculares dado que su presencia permitirá reestratificar el riesgo cardiovascular del paciente.

\section{a. Nefropatía}

La alta incidencia de DM constituye un problema para la salud mundial debido al riesgo asociado no sólo al desarrollo de ERC y de enfermedades cardiovasculares, sino también a la muerte prematura².

La DM2 podría clasificarse como integrante de los síndromes cardiorenales secundarios (tipo 5), ya que es una condición sistémica que puede causar disfunción simultánea del corazón y el riñón ${ }^{3}$.

En la actualidad ha quedado claro que sólo considerando el control glucémico no logramos abordar la compleja naturaleza e interacciones que presentan estos pacientes, constatándose que ese abordaje parcial ha generado un alto nivel de insatisfacción en cuanto al alcance de las metas terapéuticas y no ha logrado evitar o retardar la progresión de las complicaciones $^{4,5}$. Los mecanismos de progresión de daño en ambos órganos, corazón y riñón, son complejos considerando que los eventos cardiovasculares son altamente dependientes de la regulación del contenido de sodio y agua; del mismo modo, las alteraciones de los riñones dependen directamente del flujo sanguíneo y la presión generada por el corazón estableciéndose un círculo vicioso de progresivo deterioro más allá del órgano que lo inicia.

Se debe considerar que tanto el aumento de la albuminuria como la caída de la función renal se asocian en forma independiente al aumento de riesgo de todas las causas de morbimortalidad CV, tanto en la población general como en pacientes con $\mathrm{DM}^{6}$. Frente a la presencia de ERC corresponde conocer su clasificación actual para encarar las modificaciones del tratamiento de la DM.

La clasificación de la enfermedad renal se basa en la evaluación de la función renal y la albuminuria; la primera se evalúa a través de la creatinina sérica y/o las aplicaciones del aclaramiento (clearance) de creatinina o del filtrado estimado por fórmula (MDRD 4 abreviada o CKD-EPI en caso de filtrado $\leq 60 \mathrm{~mL} / \mathrm{min} / 1,73 \mathrm{~m}^{2}$ ). La albuminuria debe evaluarse desde el diagnóstico de la DM2; su recolección puede realizarse en muestra aislada de la mañana expresándose en albuminuria/creatininuria $(\mathrm{mg} / \mathrm{g}$ ) o en orina de $24 \mathrm{~h}$, y se debe confirmar con dos muestras patológicas antes de considerarse significativa para ser tratada. De obtener valores normales, solicitar en el seguimiento al menos una vez al año7. Según la evaluación clínica individualizada del paciente se podrá optar por realizar dicho control con mayor frecuencia. Durante el desarrollo de la ERC, la caída del filtrado glomerular por debajo de $60 \mathrm{~mL} / \mathrm{min} / 1,73 \mathrm{~m}^{2}$ se denomina insuficiencia renal (IR), la cual puede clasificarse en estadios 3, 4 ó 5. Si se constatan los valores en forma estable por tres meses, se considera insuficiencia renal crónica (IRC). En estas etapas progresivamente pueden aparecer otros factores de riesgo adicionales, como alteraciones metabólicas y humorales por la toxicidad urémica, anemia, desequilibrio en el metabolismo fosfocálcico y/o acidosis que repercuten también en la evolución y riesgo de morbimortalidad cardiovasculares ${ }^{8}$.

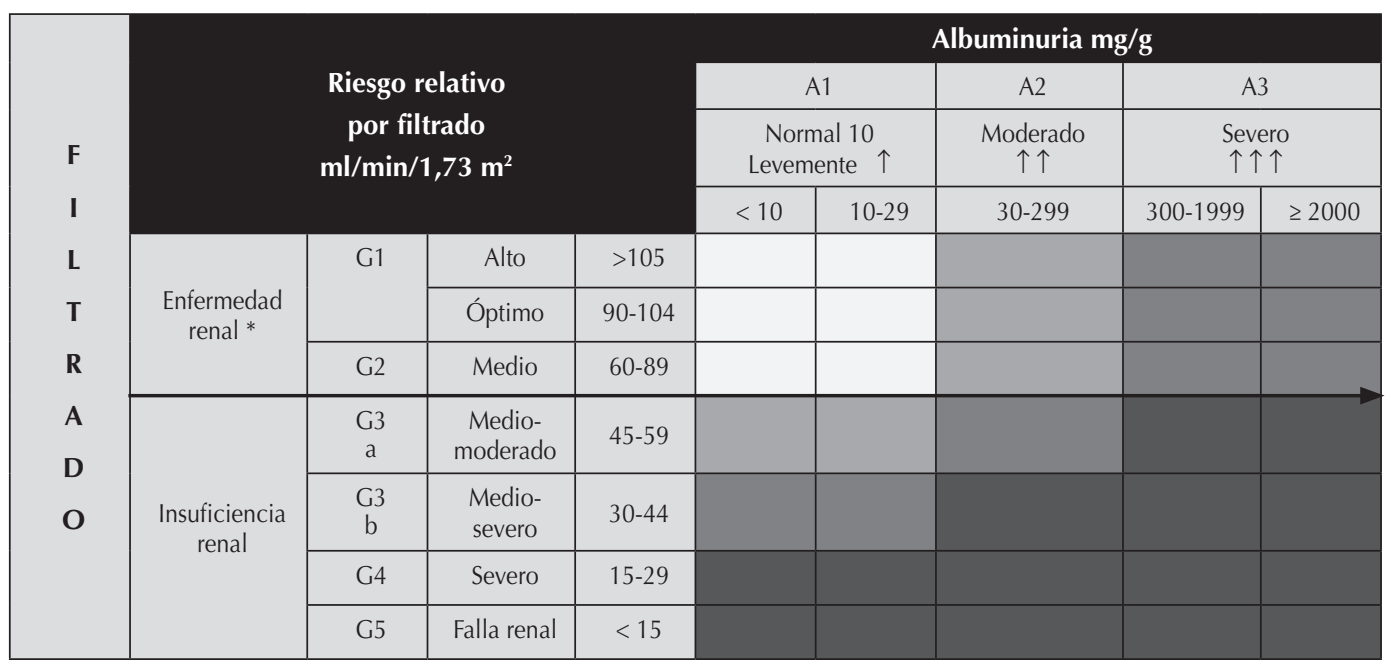

*ER se denomina a cualquier alteración del sedimento estructural o histológica

Blanco bajo, gris claro moderado, gris intermedio alto, gris oscuro altísimo aumento de riesgo cardiovascular y/o renal

Tabla 1: Estadios de clasificación de enfermedad renal. 


\section{Tratamiento de la hiperglucemia en enfermedad renal}

- Estadios 1-2: al igual que en la población general se plantean objetivos de $\mathrm{HbA} 1 \mathrm{c}<7 \%$ para evitar la progresión de las lesiones microvasculares y valores más estrictos de $\leq 6,5 \%$ en caso de pacientes sin complicaciones ni riesgo de hipoglucemia. Deberá realizarse una evaluación individualizada de la presencia de factores de riesgo y/o comorbilidades asociadas, que facilitará una mejor elección del fármaco adecuado para estos pacientes 9 .

- Estadios 3-4-5: no hay estudios aleatorizados, controlados, doble ciego en IRC que sostengan el beneficio del tratamiento intensificado, lo que plantea un debate en la actualidad de cuál es el objetivo por alcanzar en pacientes con ERC. Debemos considerar que las recomendaciones de todas las Sociedades Científicas se basan en la opinión de expertos. Se sugiere individualizar los objetivos de acuerdo con las características y vulnerabilidad del paciente. Múltiples variables modifican el perfil glucémico en pacientes con IRC: cambios metabólicos y hormonales debido a la acumulación de toxinas, factores inflamatorios y estrés oxidativo que aumentan la insulinorresistencia, así como también alteraciones en la secreción de insulina por la variación de los niveles de parathormona o vitamina $\mathrm{D}$ o de ambas. Por otra parte, la glucemia puede verse modificada según el tipo de tratamiento sustitutivo instituido (hemo- diálisis, peritoneal o los fármacos del trasplante). Además, los pacientes con IRC presentan un mayor riesgo de hipoglucemia por encontrarse disminuida la gluconeogénesis renal y/o el catabolismo de la insulina. Para los pacientes con ERC estadios 3,4 y 5 se sugiere alcanzar metas de $\mathrm{HbA} 1 \mathrm{c}<7 \%$ para minimizar el riesgo de hipoglucemia. Objetivos más conservadores pueden plantearse en pacientes vulnerables, con expectativa de vida corta o alto riesgo de hipoglucemia ${ }^{10}$. Otra dificultad que se plantea es el uso de HbA1c para el seguimiento metabólico. La anemia, que puede estar presente en los DM2 aún con función renal normal que progresa a medida que se instala la IRC, determina una hemoglobina disminuida que, junto con la ferropenia y el uso de eritropoyetina, modifica la correlación entre la glucemia y la hemoglobina alterando el valor de $\mathrm{HbA} 1 \mathrm{c}$, lo que no permite realizar un seguimiento adecuado con este método. Tampoco está avalado en IRC el uso de fructosamina ${ }^{11}$. Por ello se destaca la importancia del automonitoreo para ajustes de tratamiento ${ }^{12}$. La IR modifica, además, la farmacocinética y farmacodinamia de algunos fármacos, por lo que abordaremos los cambios que debemos considerar al utilizarlos ${ }^{13,14}$.

- Metformina: se considera el fármaco de primera elección para los pacientes con DM2 y $\mathrm{ERC}^{15}$. Se debe contraindicar su uso con un filtrado menor de $30 \mathrm{~mL} / \mathrm{min} / 1,73 \mathrm{~m}^{2}$, pero puede emplearse con precaución con filtrados de entre 30 y $45 \mathrm{~mL} / \mathrm{min} / 1,73 \mathrm{~m}^{2} 16,17$.

\begin{tabular}{|l|c|c|l|}
\hline \multicolumn{1}{|c|}{ Estadios renales } & Filtrado $\mathbf{~ m L / m i n} / \mathbf{1}, \mathbf{7 3} \mathbf{~ m}^{\mathbf{2}}$ & Dosis (diaria) & \multicolumn{1}{c|}{ Comentarios } \\
\hline 3A & $45 \mathrm{a} 60$ & $2.000 \mathrm{mg}$ & Evitar si la función renal es inestable \\
\hline 3B & $30 \mathrm{a} 45$ & $1.000 \mathrm{mg}$ & $\begin{array}{l}\text { No iniciar } \\
\text { Puede continuar su uso } \\
\text { Evitar si la función es inestable } \\
\text { Realizar seguimiento más estricto }\end{array}$ \\
\hline 4 & $15 \mathrm{a} 30$ & & No usar \\
\hline 5 & $<15$ & & No usar \\
\hline
\end{tabular}

Tabla 2: Normas sugeridas por la FDA para el uso de metformina.

- Sulfonilurea: la mayoría de las normas y algoritmos actuales ha mantenido las sulfonilureas al reconocer que son agentes de bajo costo y eficaces en la reducción de la glucemia. Sin embargo, se alerta sobre el riesgo de hipoglucemia y, en algunos casos, de un mayor riesgo cardiovascular ${ }^{18}$, a pesar de que la NKF habilita el uso de glipizida y gliclazida sin cambios en IRC sin diálisis, aunque debemos considerar el mayor riesgo de hipoglucemia en relación con la caída del filtrado. No todas las sulfonilureas son iguales: la gliclazida presenta menor riesgo de hipoglucemia comparada con otras, pero en caso de no disponer de otra opción la recomendamos preferentemente. 
- Glinidas: ofrecen menor riesgo de hipoglucemia que las sulfonilureas y menor duración de acción, pero son útiles para la hiperglucemia posprandial. Nateglinida es metabolizada por el hígado y un metabolito activo se excreta por riñón; la repaglinida no presenta metabolitos activos. En estadio de IRC, utilizar repaglinida y ajustar la dosis según la respuesta y el riesgo de hipoglucemia.

- Glitazonas: si bien son fármacos que no requie- ren ajuste en los pacientes con ERC y no aumentan el riesgo de hipoglucemia, debemos evaluar el costo/ beneficio a causa de sus efectos adversos como fracturas, retención de líquido y edemas; debe utilizarse con precaución en pacientes con proteinuria o deterioro del filtrado o en ambos casos, y contraindicada especialmente con riesgo de insuficiencia cardíaca ${ }^{19}$.

- Acarbosa: se elimina un 2\% por riñón; se sugiere evitar su uso con filtrado $<30 \mathrm{~mL} / \mathrm{min} / 1,73 \mathrm{~m}^{2}$.

\begin{tabular}{|c|c|c|c|c|c|c|}
\hline Estadios & $1-2$ & \multicolumn{2}{|c|}{$3-A$} & $3 \mathrm{~B}$ & 4 & 5 \\
\hline Filtrado $\mathrm{mL} / \mathrm{min} / 1,73 \mathrm{~m}^{2}$ & 260 & \multicolumn{2}{|c|}{$<60-45$} & $<45-30$ & $<30-15$ & $<15$ \\
\hline \multicolumn{7}{|l|}{ Metformina } \\
\hline \multicolumn{7}{|l|}{ Gumepirida } \\
\hline \multicolumn{7}{|l|}{ Glibenclamida } \\
\hline \multicolumn{7}{|l|}{ Gucazida } \\
\hline \multicolumn{7}{|l|}{ Gupizida } \\
\hline \multicolumn{7}{|l|}{ Nateglinida } \\
\hline \multicolumn{7}{|l|}{ Repaglinida } \\
\hline \multicolumn{7}{|l|}{ Acarbose } \\
\hline \multicolumn{7}{|l|}{ Proglitazona } \\
\hline \multicolumn{3}{|c|}{$\begin{array}{l}\text { Uso habitual } \\
\text { Sugerimos no usar por riesgo de } \\
\text { hipoglucemia/metabolitos activos/eliminación } \\
\text { renal/efectos adversos aumentado en } \\
\text { estos estadios }\end{array}$} & \multicolumn{4}{|c|}{$\begin{array}{l}\text { Sugerimos su uso con precaución con } \\
\text { ajuste dosis por seguridad y/o hipoglucemia } \\
\text { y/o efectos adversos }\end{array}$} \\
\hline
\end{tabular}

Tabla 3: Indicaciones de fármacos según estadio renal.

- Inhibidores DPP-4: por tratarse de fármacos seguros, sin riesgo de hipoglucemia, pueden usarse en IRC en todos los estadios ${ }^{20}$. Tienen un efecto moderado sobre la $\mathrm{HbA} 1 \mathrm{c}$; algunos han demostrado disminuir la albuminuria. Debe considerarse el ajuste de dosis que se excretan por vía urinaria en el caso de la saxagliptina, vildagliptina y sitagliptina, no así en el de linagliptina o teneligliptina que no necesitan ajuste de dosis. Debemos considerar que el estudio CARMELINA, realizado con linagliptina, incluyó pacientes con IRC constituyendo el estudio con más pacientes en estos estadios, que confirmó la eficacia y seguridad de este grupo de fármacos así como la disminución de la albuminuria ${ }^{21}$. Todos los estudios con i-DPP4 mostraron efectos neutros de no inferioridad con respecto a los resultados de eventos cardiovasculares y renales.

- Agonistas de GLP-1: liraglutida y dulaglutida son fármacos que han demostrado no sólo beneficios cardiovasculares sino también disminución significativa de albuminuria y de algunos compuestos renales. Tanto el estudio LEADER en pacientes con eventos CV previos en su mayoría como el estudio REWIND con dulaglutida en pacientes con factores de riesgo confirmaron estos beneficios ${ }^{22,23}$. 
Revista de la Sociedad Argentina de Diabetes Año 55 Vol. 55 № 1 Suplemento Consenso Sociedad Argentina de CardiologíaSociedad Argentina de Diabetes Enero-abril de 2021: 03-70 ISSN 0325-5247 (impresa) ISSN 2346-9420 (en línea)

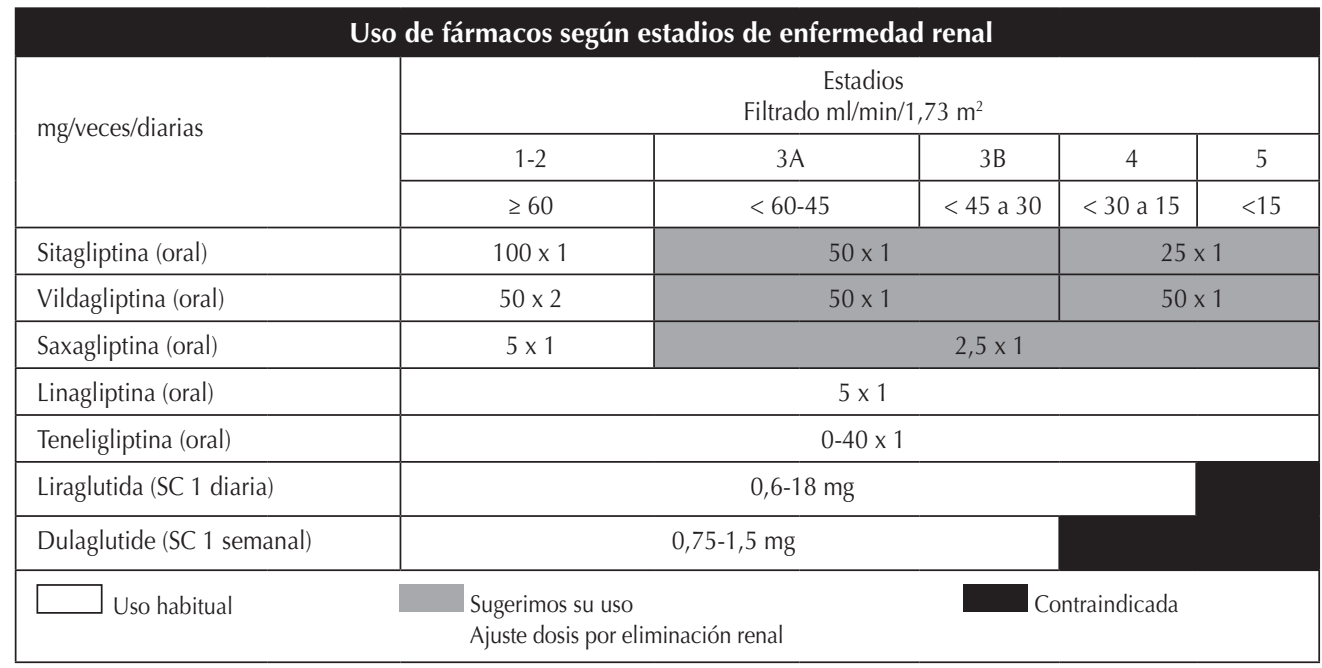

Las dosis están expresadas en mg.

Tabla 4: Dosis de incretinas según la función renal.

- Inhibidores de SGLT-2: este grupo mostró en los estudios EMPA-REG y CANVAS un efecto beneficioso en eventos cardiovasculares, así como en eventos renales tanto sobre la reducción en la caída de filtrado como en la disminución de la albuminuria $^{24,25}$. El estudio DECLARE confirmó estos beneficios en pacientes con eventos $\mathrm{CV}$, así como en aquellos con factores de riesgo ${ }^{26}$. El estudio CREDENCE evaluó canagliflozina en pacientes con IRC y albuminuria mostrando consistencia en los resultados tanto en eventos $C V$, en disminución significativa de insuficiencia cardíaca, como en protección renal y disminución de albuminuria, y mostró seguridad también en su uso en este subgrupo de pacientes ${ }^{27}$. Resultados tan contundentes plantean la elección de este grupo farmacológico en los pacientes con DM2 y ERC.

\begin{tabular}{|c|c|c|c|c|c|}
\hline \multirow{3}{*}{ Dosis mg/diaria } & \multicolumn{5}{|c|}{$\begin{array}{c}\text { Estadios } \\
\text { Filtrado } \mathrm{ml} / \mathrm{min} / 1,73 \mathrm{~m}^{2}\end{array}$} \\
\hline & $1-2$ & $3 \mathrm{~A}$ & $3 \mathrm{~B}$ & 4 & 5 \\
\hline & $\geq 60$ & $<60-45$ & $<45$ a 30 & $<30$ a 15 & $<15$ \\
\hline Dapagliflozina (oral)* & 10 & & & & \\
\hline Empaglifozina (oral) & \multicolumn{3}{|c|}{$10-25$} & & \\
\hline Canagliflozina (oral)* & $300-100$ & 100 & & & \\
\hline \begin{tabular}{|l|l}
$\square$ & Uso habitual
\end{tabular} & \multicolumn{2}{|c|}{$\begin{array}{l}\text { Sugerimos su uso } \\
\text { Ajuste dosis }\end{array}$} & & traindicada & \\
\hline
\end{tabular}

*Al momento de la publicación de este documento se encuentra avalado o en estudio por diferentes entidades regulatorias el uso con filtrados hasta $30 \mathrm{ml} / \mathrm{min}$.

Tabla 5: Dosis de los inhibidores de los SGLT-2 según la función renal.

- Insulina: en la IRC existen cambios metabólicos que alteran la sensibilidad o el requerimiento de la insulina o ambos. Se debe considerar individualizar el algoritmo glucémico que presente el paciente evitando como primer objetivo el riesgo de hipoglucemia. En caso de hallarse en tratamiento sustitutivo, deberán considerarse también las modificaciones que cada uno de los tratamientos genera en el perfil glucémico. Si bien todas las insulinas pueden usarse, si no se alcanzan los objetivos terapéuticos, se sugiere el uso de análogos por el menor riesgo de hipoglucemia en los pacientes que presenten ese riesgo (Recomendaciones Ila). 
Revista de la Sociedad Argentina de Diabetes Año 55 Vol. 55 № 1 Suplemento Consenso Sociedad Argentina de CardiologíaSociedad Argentina de Diabetes Enero-abril de 2021: 03-70 ISSN 0325-5247 (impresa) ISSN 2346-9420 (en línea)

\section{Recomendaciones}

- En pacientes con IRC estadios 3-4-5 se sugiere $\mathrm{HbA} 1 \mathrm{c}<7 \%$ minimizando el riesgo de hipoglucemia (considerar las condiciones clínicas asociadas a ER que puedan alterar la correcta interpretación de la HbA1c) (Grado de recomendación Ila. Nivel de evidencia C).

- Debido a las dificultades en la interpretación adecuada de $\mathrm{HbA} 1 \mathrm{c}$ en pacientes con IRC se sugiere considerar los valores del automonitoreo glucémico para el ajuste del tratamiento (Grado de recomendación Ila. Nivel de evidencia C).

- No se recomienda el uso de fructosamina para el seguimiento metabólico (Grado de recomendación III. Nivel de evidencia C).

- Se debe considerar el uso de i-SGLT-2 en pacientes con ECV e IRC estadio 3 debido al impacto sobre la progresión de la ER y la albuminuria (evaluar las recomendaciones locales para el uso de iSGLT-2 de acuerdo con clearance de creatinina) (Grado de recomendación I. Nivel de evidencia A).

- Se debe considerar el uso de agonistas de los receptores de GLP-1 en pacientes con ECV e IRC en estadios 3-4 debido a su impacto predominante sobre la albuminuria (Grado de recomendación I. Nivel de evidencia A).

\section{b. Neuropatía autonómica cardíaca}

La lesión del sistema nervioso en la persona con DM se considera la manifestación microangiopática de mayor prevalencia y precocidad de aparición. Debe tenerse presente que la neuropatía diabética no solamente afecta las extremidades de los pacientes, sino que, lesionando el sistema nervioso autónomo, produce compromiso en todos los órganos de la economía. La falta de criterios unificados para su diagnóstico dificulta establecer su prevalencia, pero se considera que el $90 \%$ de las personas con DM puede tener algún tipo de compromiso neuropático luego de 20 o más años de evolución de la enfermedad ${ }^{28}$. La lesión nerviosa en estos pacientes no se limita a la afectación somática; es mucho más amplia y genera la necesidad de buscar alteraciones en el sistema nervioso autónomo (SNA). Las formas sintomáticas de la neuropatía autonómica no son frecuentes pero, debido a las características anatómicas del SNA (fibras amielínicas y de pequeño diámetro), su afectación es temprana en la evolución de la enfermedad ${ }^{29}$. Dentro del compromiso autonómico, la afectación cardiovascular, es decir, la neuropatía autonómica cardíaca, requiere especial atención ${ }^{30}$. Las manifestaciones más frecuentes son la intolerancia al ejercicio por modulación simpática alterada, la frecuencia cardíaca basal elevada con ausencia de variabilidad y los síncopes secundarios a la hipotensión ortostática. También se ha asociado la neuropatía autonómica cardíaca (NAC) con un mayor riesgo de infarto de miocardio silente y muerte súbita por arritmias malignas. La falta de variabilidad del intervalo RR y el QT prolongado en la NAC son características observables en esta entidad. La hipotensión postural implica un fallo en la vasoconstricción visceral y vascular periférica, que determina afectación del sistema nervioso simpático y NAC severa. Los síntomas pueden estar exacerbados durante las comidas. El diagnóstico de la neuropatía autonómica diabética requiere métodos instrumentales auxiliares de diferente grado de complejidad. Las pruebas que evalúan la variabilidad de la frecuencia cardíaca son el método clásico para el diagnóstico de la neuropatía autonómica cardiovascular parasimpática. Poseen una buena especificidad, sensibilidad y reproducibilidad. Las más estandarizadas son la variación de la frecuencia cardíaca durante la respiración profunda (prueba que presenta especificidad $>80 \%$ ) y la variación del intervalo $R R$ durante la prueba de Valsalva y ante el cambio de decúbito. La valoración de la presión arterial ante los cambios posturales se emplea para evaluar la respuesta que tiene la inervación simpática sobre la regulación de la actividad refleja del sistema cardiovascular ${ }^{31}$. Deben tenerse presentes variables como hidratación, medicación y arritmias cardiovasculares que pueden modificar los resultados de las pruebas. Otros métodos, como el análisis del intervalo RR en el dominio frecuencia y tiempo, y la evaluación de la inervación simpática a través de $P E T$, requieren procedimientos más sofisticados para establecer el diagnóstico. Detectada la NAC, corresponde un tratamiento cuidadoso del paciente. En lo que respecta a su control diabetológico, se buscará evitar las hipoglucemias por su potencial efecto disparador de arritmias y otros eventos coronarios. Existen algunas evidencias de un efecto positivo del ácido alfa lipoico sobre la variabilidad de la frecuencia cardíaca ${ }^{32}$. Se deberán dar consejos para evitar maniobras de Valsalva o cambios posturales bruscos. Ante la posibilidad de NAC, se sugiere evaluar la presencia de isquemia miocárdica silente. 
Revista de la Sociedad Argentina de Diabetes Año 55 Vol. 55 № 1 Suplemento Consenso Sociedad Argentina de CardiologíaSociedad Argentina de Diabetes Enero-abril de 2021: 03-70 ISSN 0325-5247 (impresa) ISSN 2346-9420 (en línea)

\section{BIBLIOGRAFÍA}

1. Juutilainen A, Lehto S, Ronnemaa T, Pyorala K, Laakso M. Retinopathy predicts cardiovascular mortality in type 2 diabetic men and women: Response toTargher et al. Diabetes Care 2007; 30(6):e52-e52.

2. Sattar N, Rawshani A, Franzén S, Rawshani A, Svensson AM, Rosengren A. Age at diagnosis of type 2 diabetes mellitus and associations with cardiovascular and mortality risks. Circulation 2019; 139(19):2228-2237.

3. Rangaswami J, Bhalla V, Blair JEA, Chang TI, Costa S, Lentine $\mathrm{KL}$, et al. Cardiorenal syndrome: classification, pathophysiology, diagnosis, and treatment strategies: a scientific statement from the American Heart Association. Circulation 2019; 139(16):e840-e878.

4. Li S, Wang J, Zhang B, Li X, Liu Y. Diabetes mellitus and causespecific mortality: A population-based study. Diabetes Metab J 2019; 43(3):319-341.

5. McGurnaghan S, Blackbourn LAK, Mocevic E, Haagen Panton U R, McCrimmon J, Sattar N, et al. Research: complications cardiovascular disease prevalence and risk factor prevalence in type 2 DM: a contemporary analysis. Diabet Med 2019; 36: 718-725

6. National Kidney Foundation. KDOQI clinical practice guidelines and clinical practice recommendations for DM and chronic kidney disease. Am J Kidney Dis 2007; 49(suppl 2):S12-S154.

7. American DM Association. Microvascular complications and foot care: standards of medical care in DM. Diabetes Care 2019; 42(Suppl. 1):S124-S138.

8. Alicic RZ, Rooney MT,Tuttle KR. Diabetic kidney disease challenges, progress, and possibilities. Clin J Am Soc Nephrol 2017; 12:2032-2045.

9. American DM Association. Glycemic targets: Standards of medical care in DM. Diabetes Care 2019; 42(Suppl.1):S61-S70.

10. Davies MJ, D'Alessio DA, Fradkin J, Mathieu C, Kernan W, Mingroneet $G$, et al. Management of hyperglycemia in type 2 DM 2018. A consensus report by the American DM Association (ADA) and the European Association for the Study of DM (EASD). Diabetes Care 2018; 41:2669-2701.

11. Chen HS, Wu TE, Lin HD, Jap TS, Hsiao LC, Lee SH, et al. Hemoglobin $A(1 c)$ and fructosamine for assessing glycemic control in diabetic patients with CKD stages 3 and 4 . Am J Kidney Dis 2010; 55(5):867-74.

12. Roussel R, Lorraine J, Rodríguez A, Salaun-Martin C. Overview of data concerning the safe use of antihyperglycemic medications in type 2 diabetes mellitus and chronic kidney disease. Adv Ther 2015; 32(11):1029-64.

13. Lo C, Toyama T, Wang Y, Lin J, Hirakawa Y, Jun M, et al. Insulin and glucose-lowering agents for treating people with diabetes and chronic kidney disease. Cochrane Database Syst Rev 2018; 9:CD011798.

14. Perkovic V, Agarwal R, Fioretto P, Hemmelgarn BR, Levin A, Thomas MC, et al Management of patients with DM and CKD: conclusions from a "Kidney Disease: Improving Global Outcomes" (KDIGO) Controversies Conference. Kidney Int. 2016; 90(6):1175-1183.

15. American Diabetes Association. Pharmacologic approaches to glycemic treatment: Standards of medical care in DM 2019. Diabetes Care 2019; 42(Suppl. 1):S90-S102

16. Bakris GL, Molitch ME. Should restrictions be relaxed for metformin use in chronic kidney disease? Yes, they should be relaxed! What's the fuss? Diabetes Care 2016; 39:1287-1291.

17. Kamyar Kalantar-Zadeh, Csaba P, Kovesdy CP. Should restrictions be relaxed for metformin use in chronic kidney disease? No, we should never again compromise safety. Diabetes Care 2016; 39:1281-1286.

18. Douros A, Dell'Aniello S, Yu OHY, Filion KB, Azoulay L, Suissa $\mathrm{S}$. Sulfonylureas as second line drugs in type $2 \mathrm{DM}$ and the risk of cardiovascular and hypoglycaemic events: population based cohort study. BMJ 2018; 362:k2693.
19. Lincoff AM, Wolski K, Nicholls SJ, Nissen SE. Pioglitazone and risk of cardiovascular events in patients with type 2 diabetes mellitus: a meta-analysis of randomized trials JAMA 2007; 298(10):1180-8.

20. Scheen AJ, Delanaye P. Review Renal outcomes with dipeptidyl peptidase-4 inhibitors. Diabetes \& Metabolism 2018; 44:101-111.

21. Hanssen NM, Jandeleit-Dahm KA. Dipeptidyl peptidase-4 inhibitors and cardiovascular and renal disease in type $2 \mathrm{DM}$ : What have we learned from the CARMELINA trial? Diab Vasc Dis Res 2019; 16(4):303-309.

22. Marso SP, Daniels GH, Brown-Frandsen K, Kristensen P, Mann JFE, Naucket MA, et al. Liraglutide and cardiovascular outcomes in type 2 DM. N Engl J Med 2016; 375:311-322.

23. Gerstein HC, Colhoun HM, Dagenais GR, Díaz R, Lakshmanan M, Pais P, Probstfield J, et al. Dulaglutide and cardiovascular outcomes in type 2 DM (REWIND): a double-blind, randomised placebo-controlled trial. Lancet 2019 Jul 13; 394(10193):121-130.

24. Wanner C, Inzucchi SE, Lachin JM, Fitchett D, von Eynatten M, Mattheus $M$, et al. Empagliflozin and progression of kidney disease in type 2 DM. N Engl J Med 2016; 375(4):323-34.

25. Butler J, Zannad F, Fitchett D, Zinman B, Koitka-Weber A, von Eynatten M, et al. Empagliflozin improves kidney outcomes in patients with or without heart failure. Circ Heart Fail 2019; 12(6):e005875.

26. Mosenzon O, Wiviott SD, Cahn A, Rozenberg A, Yanuv I, Goodrich $E L$, et al. Effects of dapagliflozin on development and progression of kidney disease in patients with type 2 DM: an analysis from the DECLARE-TIMI 58 randomised trial. Lancet Diabetes Endocrinol 2019; 7(8):606-617.

27. Perkovic $\mathrm{V}$, Jardine MJ, Neal B, Bompoint S, Heerspink HJL, Charytan DM, et al. Canagliflozin and renal outcomes in type $2 \mathrm{DM}$ and nephropathy. CREDENCE Trial Investigators. N Engl J Med 2019; 380(24):2295-2306.

28. Vinik A. Diabetic neuropathies. Med Clin North Am 2004 88(4):947-949.

29. Rosas-Guzmán J, Odriozola A, Davidson JA, Costa Gil J, Fuente G, García de los Ríos M, et al. Guía práctica en el manejo de la polineuropatía diabética. Revista de la Asociación Latinoamericana de Diabetes 2010; 8(Sup.1).

30. Jadzinsky M, Fuente G. Neuropatía diabética periférica y autonómica. En: Diabetes Mellitus $4^{\circ}$ edición. Editor: Ruiz M. 2011 Cap. 32:595-612.

31. Lefaucheur JP, Wahab A, Planté-Bordeneuve V, Sène D, MénardLefaucheur I, Rouie D, et al. Diagnosis of small fiber neuropathy: A comparative study of five neurophysiologycal tests. Neurophysiol Clin 2015; 45(6):445-55.

32. Ziegler D, Nowak H, Kempler P, Vargha P, Low PA. Treatment of symptomatic diabetic polyneuropathy with the antioxidant alphalipoic acid: a meta-analysis. Diabet Med 2004; 21(2):114-12.

\section{Abreviaturas}

AAS: ácido acetilsalicílico

ACV: accidente cerebrovascular

AF: actividad física

AG n-3: ácidos grasos poliinsaturados omega-3

AIT: accidente isquémico transitorio

ARA II: antagonistas de los receptores de angiotensina II

arGLP-1: agonistas del receptor GLP-1

ATC: angioplastia transluminal coronaria

BMS: stent metálico

C-LDL: colesterol asociado a lipoproteínas de baja densidad

C-HDL: colesterol asociado a lipoproteínas de alta densidad

CRM: cirugía de revascularización miocárdica 
Revista de la Sociedad Argentina de Diabetes Año 55 Vol. 55 Nº 1 Suplemento Consenso Sociedad Argentina de CardiologíaSociedad Argentina de Diabetes Enero-abril de 2021: 03-70 ISSN 0325-5247 (impresa) ISSN 2346-9420 (en línea)

\section{CV: cardiovascular}

DES: stent farmacológico

DHA: ácido docosahexaenoico

DM: diabetes mellitus

EAMI: enfermedad arterial de miembros inferiores

ECV: enfermedad cardiovascular

EMV: enfermedad de múltiples vasos

EPA: ácido eicosapentaenoico

ERC: enfermedad renal crónica

FA: fibrilación auricular

FEVI: fracción de eyección del ventrículo izquierdo

GPA: glucemia plasmática de ayunas

HbA1c: hemoglobina glicosilada A1C

HDA: hemorragia digestiva alta

HDB: hemorragia digestiva baja

HVI: hipertrofia ventricular izquierda

HTA: hipertensión arterial

IAM: infarto agudo de miocardio

IC: insuficiencia cardíaca

ICMI: isquemia crítica de miembros inferiores

IECA: inhibidores de la enzima convertidora de angiotensina

IMC: índice de masa corporal

¡PCSK9: inhibidores de la enzima proproteína convertasa subtilisina kexina tipo 9

iSGLT-2: inhibidores del cotransoportador renal SGLT-2
IR: insuficiencia renal

IRC: insuficiencia renal crónica

MAPA: monitoreo ambulatorio de la presión arterial

MACE: eventos adversos cardiovasculares mayores

NAC: neuropatía autonómica cardíaca

PA: presión arterial

PAS: presión arterial sistólica

PCSK9: proproteína convertasa subtilisina kexina tipo 9

PTOG: prueba de tolerancia oral a la glucosa

QM: quilomicrones

rLDL: receptor C-LDL

$R R R$ : reducción de riesgo relativo

SAC: Sociedad Argentina de Cardiología

SAD: Sociedad Argentina de Diabetes

SAHOS: síndrome de apnea/hipopnea obstructiva del sueño

SCA: síndrome coronario agudo

SCACEST: síndrome coronario agudo con elevación del ST

SCASEST: síndrome coronario agudo sin elevación del ST

SM: síndrome metabólico

SNA: sistema nervioso autónomo

SU: sulfonilureas

TAG: tolerancia alterada a la glucosa

TG: triglicéridos

TN: terapia nutricional

VCT: valor calórico total

\section{ANEXO I}

\section{Fisiopatología de la dislipemia en DM}

El riesgo de morbimortalidad cardiovascular en el paciente con diabetes mellitus tipo 2 (DM2) es mayor que en sujetos sanos ${ }^{1-2}$. En esta población, los parámetros lipídicos son los principales determinantes del riesgo cardiovascular $(\mathrm{CVC})^{3-4}$. En esta patología metabólica, las alteraciones lipídicas son tanto de tipo cuantitativas como cualitativas ${ }^{5-6}$. Por anormalidades cuantitativas nos referimos, por ejemplo, al aumento de los niveles plasmáticos de triglicéridos o a la disminución de la concentración del colesterol transportado por las lipoproteínas de alta densidad (C-HDL), y por cualitativas al aumento de la proporción de lipoproteínas de baja densidad pequeñas y densas, altamente aterogénicas, a la alteración de la composición química de las lipoproteínas circulantes y a la gluco-oxidación de las apoproteínas.

Existe una elevada prevalencia de dislipemias en los pacientes con DM. En pacientes con DM2, la frecuencia oscila del 72 al $85 \%^{7}$, y en DM1 es de aproximadamente $35 \%{ }^{8}$. La dislipemia del paciente con DM posee las siguientes características:

- Aumento de los niveles de las lipoproteínas ricas en triglicéridos, tanto de producción hepática (lipoproteínas de muy baja densidad o VLDL) como de producción intestinal en estado posprandial (quilomicrones, QM).

- Disminución de la concentración de C-HDL.

- Aumento de la proporción de lipoproteínas de baja densidad (LDL) pequeñas y densas ${ }^{5}$.

Es importante recordar las acciones fisiológicas de la insulina sobre la supresión de la proteína microsomal de transferencia de triglicéridos, proteína clave en el ensamblaje de las lipoproteínas ricas en triglicéridos, mecanismo demostrado en sujetos sanos ${ }^{9}$. En pacientes diabéticos, a nivel hepático, existe resistencia a la acción inhibitoria de la insulina sobre la producción de lipoproteínas ricas en triglicéridos ${ }^{10}$ y lo mismo ocurre a nivel intestinal. Esto causa que la acción de la insulina sobre la producción de QM esté disminuida o au- 
sente, y se genere el aumento de la lipemia posprandial en el paciente diabético"11.

Respecto de las LDL, su catabolismo está disminuido, en parte porque la glicosilación no enzimática del componente proteico de esas lipoproteínas causa una menor afinidad por su receptor ${ }^{12}$. Además, el hiperinsulinismo genera menor expresión del receptor de $\mathrm{LDL}^{13}$. El predominio de las LDL pequeñas y densas en los pacientes DM2, conocido como fenotipo $B$, se debe a la hipertrigliceridemia que estimula el intercambio de triglicéridos y colesterol entre las lipoproteínas ricas en triglicéridos y las LDL por acción de la proteína transportadora de colesterol esterificado (CETP, sus siglas en inglés) $)^{14,15}$.

La hipertrigliceridemia, por mecanismos similares, disminuye los niveles de HDL y hace que los triglicéridos se acumulen en esta fracción lipídica. Esto genera mayor proporción de HDL2, más susceptibles a la acción de la lipasa hepáti$\mathrm{ca}^{16}$, lo cual determina un mayor catabolismo de las HDL. A su vez, el mencionado enriquecimiento en triglicéridos disminuye la capacidad de las HDL para promover la salida de colesterol de los tejidos periféricos y así reduce su carga de colesterol ${ }^{17}$.

\section{Estudio del perfil lipídico en el paciente con DM}

El estudio del perfil lipídico comprende la determinación de los niveles plasmáticos de triglicéridos, colesterol total, colesterol contenido en las LDL (C-LDL), colesterol en las HDL (C-HDL), así como el cálculo del C-no HDL. Adicionalmente, la medición de la concentración de la apoproteína $B$ podría resultar de gran utilidad, dado que su aumento refleja un incremento del número de lipoproteínas aterogénicas en circulación ${ }^{18}$.

A favor del empleo de la apoproteína B y del C-no $\mathrm{HDL}$, cabe destacar que la medición de los niveles de C-LDL por los métodos disponibles en la actualidad posee coeficientes de variación elevados. A su vez, su cálculo mediante la fórmula de Friedewald (C-LDL=colesterol total-triglicéridos/5+C-HDL) presenta desviaciones frente a niveles altos de triglicéridos o bajos de C-LDL, sumándose a ello la variabilidad metodológica de cada uno de los parámetros que la componen y la variabilidad biológica particularmente elevada de los triglicéridos (aproximadamente 20\%). Finalmente, hay que tener en cuenta que la apoproteína B y el C-no HDL no brindan la misma información, sino que son parámetros complementarios.
Siguiendo los lineamientos de la Sociedad Europea de Aterosclerosis y de la Federación Europea de Química Clínica y Medicina del Laboratorio ${ }^{19}$, se recomienda que el estudio de lípidos se efectúe sin ayuno previo. Entre las ventajas se destacan, en términos generales, mayor practicidad para el paciente y los laboratorios, y mejora del valor predictivo de los triglicéridos medidos sin ayuno para eventos CVC futuros. En particular, en el caso de los pacientes diabéticos, se minimiza el riesgo de las hipoglucemias, fundamentalmente en aquellos bajo tratamientos hipoglucemiantes, y evita que el ayuno enmascare la hipertigliceridemia característica de la DM.

Cuantitativamente, la dislipemia aterogénica del paciente diabético se caracterizaría por niveles plasmáticos de triglicéridos (sin ayuno) mayores o iguales a $175 \mathrm{mg} / \mathrm{dL}$, de C-HDL menores a $40 \mathrm{mg} / \mathrm{dL}$ en hombres y a $50 \mathrm{mg} / \mathrm{dL}$ en mujeres, de C-LDL normales o ligeramente elevados y de C-no HDL mayores o iguales a $130 \mathrm{mg} / \mathrm{dL}$. En el caso de la apoproteína $B$, los valores óptimos oscilan entre menores a 65 y a $90 \mathrm{mg} / \mathrm{dl}$, de acuerdo al riesgo del paciente.

\section{BIBLIOGRAFÍA}

1. Norgaard ML, Andersen SS, Schramm TK, et al. Changes in short and long-term cardiovascular risk of incident diabetes and incident myocardial infarction. A nation wide study. Diabetologia 2010; 53(8):1612-1619. DOI:10.1007/s00125-010-1783-z.

2. Mulnier HE, Seaman HE, Raleigh VS, et al. Risk of myocardial infarction in men and women with type 2 diabetes in the UK: a cohort study using the General Practice Research Database. Diabetologia 2008; 51(9):1639-1645. DOI:10.1007/s00125-008-1076-y.

3. Eliasson B, Cederholm J, Eeg-Olofsson K, et al. Clinical usefulness of different lipid measures for prediction of coronary heart disease in type 2 diabetes: a report from the Swedish $\mathrm{Na}$ tional Diabetes Register. Diabetes Care 2011; 34(9):2095-2100. DOI:10.2337/dc11-0209.

4. Turner RC, Millns H, Neil HAW, et al. Risk factors for coronary artery disease in non-insulin dependent diabetes mellitus: United Kingdom Prospective Diabetes Study (UKPDS: 23) BMJ 1998; 316:823-828.

5. Vergès B. New insight into the pathophysiology of lipid abnormalities in type 2 diabetes. Diabetes Metab 2005; 31(5):429-439.

6. Vergès $B$. Lipid disorders in type 1 diabetes. Diabetes Metab 2009; 35(5):353-360. DOI:10.1016/j.diabet.2009.04.004.

7. Doucet J, Le Floch J-P, Bauduceau B, Verny C; SFD/SFGG Intergroup. GERODIAB: Glycaemic control and 5-year morbidity/ mortality of type 2 diabetic patients aged 70 years and older: 1. Description of the population at inclusion. Diabetes Metab 2012; 38(6):523-530. DOI:10.1016/j.diabet.2012.07.001.

8. Margeirsdottir HD, Larsen JR, Brunborg C, Overby NC, Dahl-Jørgensen K; Norwegian Study Group for Childhood Diabetes. High prevalence of cardiovascular risk factors in children and adolescents with type 1 diabetes: a population-based study. Diabetologia 2008; 51(4):554-561. DOI:10.1007/s00125-007-0921-8.

9. Pavlic M, Xiao C, Szeto L, Patterson BW, Lewis GF. Insulin acutely inhibits intestinal lipoprotein secretion in humans in part by suppressing plasma free fatty acids. Diabetes 2010; 59(3):580-587. DOI:10.2337/db09-1297. 
Revista de la Sociedad Argentina de Diabetes Año 55 Vol. 55 № 1 Suplemento Consenso Sociedad Argentina de CardiologíaSociedad Argentina de Diabetes Enero-abril de 2021: 03-70 ISSN 0325-5247 (impresa) ISSN 2346-9420 (en línea)

10. Malmström R, Packard CJ, Caslake M, et al. Defective regulation of triglyceride metabolism by insulin in the liver in NIDDM. Diabetologia 1997; 40(4):454-462. DOI:10.1007/s001250050700.

11. Nogueira JP, Maraninchi M, Béliard S, et al. Absence of acute inhibitory effect of insulin on chylomicron production in type 2 diabetes. Arterioscler Thromb Vasc Biol 2012; 32(4):1039-1044. DOI:10.1161/ATVBAHA.111.242073.

12. Witztum JL, Mahoney EM, Branks MJ, Fisher M, Elam R, Steinberg $D$. Nonenzymatic glucosylation of low-density lipoprotein alters its biologic activity. Diabetes 1982; 31(4 Pt 1):283-291. DOI:10.2337/diab.31.4.283.

13. Duvillard L, Florentin E, Lizard G, et al. Cell surface expression of LDL receptor is decreased in type 2 diabetic patients and is normalized by insulin therapy. Diabetes Care 2003; 26(5):15401544. DOI:10.2337/diacare.26.5.1540.

14. Goff DC, D'Agostino RB, Haffner SM, Otvos JD. Insulin resistance and adiposity influence lipoprotein size and subclass concentrations. Results from the Insulin Resistance Atherosclerosis Study. Metab Clin Exp 2005; 54(2):264-270. DOI:10.1016/j. metabol.2004.09.002.

15. Chan DC, Barret PHR, Watts GF. Lipoprotein transport in the metabolic syndrome: pathophysiological and interventional studies employing stable isotopy and modelling methods. Clinical Science 2004; 107(3):233-249.
16. Verges B, Brun JM, Vaillant G, et al. Influence of obesity and hypertriglyceridaemia on the low HDL2-cholesterol level and on its relationship with prevalence of atherosclerosis in type 2 diabetes. Diabetes Metab 1992; 18(4):289-297.

17. Brites FD, Cavallero E, de Geitere C, Nicolaïew N, Jacotot B, Rosseneu M, Fruchart JC, Wikinski RL, Castro GR. Abnormal capacity to induce cholesterol efflux and a new LpA-I pre-beta particle in type 2 diabetic patients. Clin Chim Acta 1999; 279(12):1-14.

18. Castillo-NúñezY, Aguilar-Salinas CA, Mendivil-Anaya CO, Rodríguez M, Lyra R. Consenso del Grupo de Tareas de la Asociación Latinoamericana de Diabetes (ALAD) sobre el diagnóstico y manejo de la dislipidemia diabética. Rev ALAD 2018; 8:118-40.

19. Nordestgaard BG, Langsted A, Mora S, Kolovou G, Baum H, Bruckert E, Watts GF, Sypniewska G, Wiklund O, Borén J, Chapman MJ, Cobbaert C, Descamps OS, von Eckardstein A, Kamstrup PR, Pulkki K, Kronenberg F, Remaley AT, Rifai N, Ros E, Langlois $\mathrm{M}$. Fasting is not routinely required for determination of a lipid Irofile: clinical and laboratory implications including flagging at desirable concentration cutpoints. A joint Consensus Statement from the European Atherosclerosis Society and European Federation of Clinical Chemistry and Laboratory Medicine. Clin Chem 2016; 62(7):930-46.

\section{ANEXO II}

\section{Algoritmo para el manejo de la hiperglucemia en pacientes hospitalizados}

\section{- En unidad de cuidados críticos}

Se sugiere iniciar bomba de infusión de insulina intravenosa (regular o análogos rápidos) a partir de $180 \mathrm{mg} / \mathrm{dl}$ con el objetivo glucémico rango 140$180 \mathrm{mg} / \mathrm{dl}$ para aquellos pacientes en cuidados críticos. Para ello es fundamental disponer de protocolos estandarizados del manejo de la infusión como así también protocolos adecuados para la prevención y tratamiento de la hipoglucemia. La infusión endovenosa (EV) debe iniciarse cuando no se alcance el objetivo glucémico y se descontinuará cuando el paciente inicie alimentación oral.

\section{Protocolo de infusión EV de insulina para pacientes críticos internados en unidad de cuidados intensivos:}

Objetivo general: glucemia 140-180 mg/dl.

1) Preparación: insulina o análogos de acción rápida: $100 \mathrm{UI}$ en $100 \mathrm{ml}$ de solución 0,9\% $\mathrm{NaCl}$ administrados por vía EV.

2) Bolo inicial y tasa de infusión de insulina: iniciar la infusión si el paciente presenta valor de glucemia $>180 \mathrm{mg} / \mathrm{dl}$. Para el cálculo dividir la glucemia inicial por 100. Dicho valor sugiere el bolo inicial EV y la tasa de infusión inicial. Por ejemplo, glucemia inicial $250 \mathrm{mg} / \mathrm{dl} ; 250 \% 100=2,5 \mathrm{u}$. Bolo inicial: 2,5 Ul luego infusión a 2,5 u/h.

3) Ajuste de la infusión: a continuación, figura el algoritmo de ajuste de la infusión en tratamiento EV con insulina (modificado de Watts):

\begin{tabular}{|c|c|c|c|c|c|c|c|}
\hline \multicolumn{2}{|c|}{ Algoritmo 1 } & \multicolumn{2}{c|}{ Algoritmo 2 } & \multicolumn{2}{c|}{ Algoritmo 3 } & \multicolumn{2}{c|}{ Algoritmo 4 } \\
\hline Glucemia & U/h & Glucemia & U/h & Glucemia & U/h & Glucemia & U/h \\
\hline$<110$ & Suspender & $<110$ & Suspender & $<110$ & Suspender & $<110$ & Suspender \\
\hline $110-119$ & 0,5 & $110-119$ & 1 & $110-119$ & 2 & $110-119$ & 3 \\
\hline $120-149$ & 1 & $120-149$ & 1.5 & $120-149$ & 3 & $120-149$ & 5 \\
\hline $150-179$ & 1,5 & $150-179$ & 2 & $150-179$ & 4 & $150-179$ & 7 \\
\hline $180-209$ & 2 & $180-209$ & 3 & $180-209$ & 5 & $180-209$ & 9 \\
\hline $210-239$ & 2 & $210-239$ & 4 & $210-239$ & 6 & $210-239$ & 12 \\
\hline $240-269$ & 3 & $240-269$ & 5 & $240-269$ & 8 & $240-269$ & 16 \\
\hline $270-299$ & 3 & $270-299$ & 6 & $270-299$ & 10 & $270-299$ & 20 \\
\hline $300-329$ & 4 & $300-329$ & 7 & $300-329$ & 12 & $300-329$ & 24 \\
\hline $300-329$ & 4 & $300-329$ & 8 & $300-329$ & 14 & $330-359$ & 28 \\
\hline$<360$ & 6 & $<360$ & 12 & $<360$ & 16 & $<360$ & 32 \\
\hline
\end{tabular}


Se continuará el rango de infusión de acuerdo a la columna de algoritmo 1 en la mayoría de los pacientes. En casos seleccionados se sugiere continuar la infusión directamente en el algoritmo 2: pacientes que se someterán a cirugía de bypass coronario, trasplante de órgano o de pancreáticos, en tratamiento con glucocorticoides a altas dosis o aquellos que previamente requerían más de $80 \mathrm{UI}$ de insulina para su tratamiento.

4) Cambio de un algoritmo a otro. Si el algoritmo no logró control glucémico y las glucemias no se encuentran dentro del objetivo buscado o si la glucemia no descendió al menos $60 \mathrm{mg} / \mathrm{dl}$ dentro de la hora de realizado el ajuste, se sugiere pasar a los algoritmos siguientes. En tanto si la glucemia es $<110 \mathrm{mg} / \mathrm{dl}$, se sugiere descender nivel de infusión.

5) Monitoreo glucémico: glucemia capilar una vez por hora hasta alcanzar el objetivo, luego puede espaciarse a cada $2 \mathrm{~h}$ si se mantienen estables las glucemias y las condiciones clínicas del paciente.

6) Tratamiento de la hipoglucemia (glucemia $<60 \mathrm{mg} / \mathrm{dL}$ ). Discontinuar la infusión de insulina y administrar glucosado hipertónico (dextrosa 50\%) endovenoso. Glucemia plasmática 40-60 mg/dl: $12,5 \mathrm{~g}$. Glucemia plasmática $<40 \mathrm{mg} / \mathrm{dl}: 25 \mathrm{~g}$. Chequear nuevamente la glucemia plasmática cada 15-30 minutos y repetir la infusión de dextrosa EV si se mantiene baja. Reinstaurar el goteo de insulina cuando la glucemia es $>80 \mathrm{mg} / \mathrm{dl}$ en dos oportunidades. Se reinicia con un algoritmo inferior al que debió suspenderse.

\section{- En cuidados intermedios y/o piso (paciente inicia alimentación oral)}

Si el paciente se encontraba bajo infusión de insulina en goteo continuo deben calcularse los requerimientos de las últimas 6 horas (h) para estimar la dosis de insulina total diaria (DTD). Un algoritmo propuesto es multiplicar por 4 el requerimiento de insulina en bomba de las últimas $6 \mathrm{~h}$ para obtener la DTD. El 50\% de esta dosis de insulina se distribuirá en dos o tres dosis de insulina NPH o en una dosis de insulina análogos lentos como insulina glargina la cual se administrará $2 \mathrm{~h}$ previas a la suspensión del goteo de insulina. El 40\% restante puede distribuirse como insulina rápida preprandial si el paciente tiene una ingesta oral adecuada y completa. Ante dudas en la ingestión pueden indicarse insulinas rápidas según la tabla de correcciones (siempre asociado a las insulinas basales). Por ejemplo, cálculo de la DTD: si el requerimiento de insulina endovenosa en goteo continuo fue de 6 u en las últimas 6 h, se calcula $6 \mathrm{u} \times$ 4: $24 \mathrm{u}$ de DTD. Del mismo se toma el 50\% como insulina basal: en el ejemplo $12 \mathrm{u}$ de insulina glargina en una dosis. El 40\% restante de la DTD se distribuye entre las cuatro comidas 2 u pre desayuno, almuerzo, merienda y cena.

\section{- Al egreso}

- Paciente con diagnóstico hecho en internación: se evaluará inicio de tratamiento de acuerdo a algoritmos de manejo de hiperglucemia del paciente con DM y eventos cardiovasculares sugiriéndose la utilización precoz de iSGLT-2 o arGLP-1 con beneficio cardiovascular.

- Paciente con diagnóstico de diabetes previo al cuadro coronario: se individualizará si continúa con insulinoterapia o reinicia tratamientos antihiperglucemiantes previos. Considerar agregar o reemplazar algunos de los fármacos por aquellos con beneficios cardiovasculares como iSGLT-2 o ar GLP-1. 